\title{
Quantum Field Theory, Grassmannians, and Algebraic Curves
}

\author{
Edward Witten * \\ Joseph Henry Laboratories, Princeton University, Princeton, NJ 08544, USA
}

\begin{abstract}
This paper is devoted in part to clarifying some aspects of the relation between quantum field theory and infinite Grassmannians, and in part to pointing out the existence of a close analogy between conformal field theory on Riemann surfaces and the modern theory of automorphic representations. Along the way we develop a multiplicative analog of the usual additive Ward identities of current algebra. We also reformulate the additive Ward identities in a way which may be useful, in terms of the residues of operator-valued differential forms. A concluding section is devoted to some remarks on string field theory. In an appendix, we attempt to clarify the recent construction by Beilinson, Manin, and Schechtman of what might be called global Virasoro algebras.
\end{abstract}

The present paper consists of several sections. In Sects. (1) and (2), I will attempt to describe in physical terminology some aspects of the relation, surveyed in [1], between Riemann surfaces and infinite Grassmannians. This relation has been essential in recent studies of the Schottky problem $[2,3]$, and its relation with quantum field theory and string theory have been the subject of recent discussions [4-6] from a physical viewpoint. In the first section we will consider the Grassmannian of [1] as the space of boundary conditions on the $\bar{D}$ operator. This way of looking at things really provides the essential link between Grassmannians and the theory of free fermions. In the second we introduce "multiplicative Ward identities." These are needed to describe the relation between the Baker function and the tau function. They also, I believe, shed considerable light on the whole phenomenon of bosonization of fermions. And they are a needed preliminary for the latter part of the paper.

In the third section, we reformulate the Ward identities of conformal field theory, first described in [14], in terms of "operator valued differential forms," which will have already made their appearance in Sect. one. In particular, we will

* Supported in part by NSF Grants PHY80-19754 and 86-16129 
show that the basic Ward identity is the statement that "the sum of the operatorvalued residues of an operator-valued differential form is zero." Apart from other possible merits, the description of conformal field theory in these terms has the virture that it makes sense purely algebraically, with ordinary Riemann surfaces replaced by curves over an arbitrary ground field. Thus, we will go on in Sects. four and five to develop the rudiments of an algebraic description of conformal field theory in the simplest cases of free fermions and of current algebra on a Riemann surface. In doing so, we will find a close relationship between conformal quantum field theory on Riemann surfaces and the mathematical theory of "automorphic representations of adèle groups" (see [7] for introductions). Thus a general quantum field theory observable is an arbitrary finite product

$$
\prod_{i=1}^{N} O_{i}\left(P_{i}\right)
$$

of local operators $O_{i}$ inserted at points $P_{i}$ on a Riemann surface. The space of such observables forms what would in the mathematical theory be called an automorphic representation (of the current algebra or Virasoro algebra appropriate to the quantum field theory in question). The Ward identities of quantum field theory assert the existence of a vector invariant under the global group (or Lie algebra), a standard condition in the theory of automorphic representations.

Section six of the paper is devoted to a brief exploration of the possibility that the "field variable" of string field theory should actually be not a string field, as it is usually taken to be, but an arbitrary product of local observables, as in Eq. (1). The discussion will be intriguing but inconclusive.

Finally, in an appendix, we express in physical language the global generalization of the Virasoro algebra which was presented recently in [19], and use it to describe the Virasoro analogues of some constructions carried out in the body of the paper for current algebra and for free fermions.

\section{Grassmannians and Determinants}

In attempting to elucidate certain of the ideas expounded in [1], we will essentially be concerned with some properties of the determinant of the Dirac operator on a Riemann surface with boundaries or punctures. The mathematical literature on fermion determinants was started in [8-10] and has a counterpart, of course, in the physics literature on effective actions and anomalies.

To be specific, consider a one component chiral fermion $\psi$ propagating on a $\operatorname{disc} D$. We take $D$ to be the region $|z| \leqq 1$, with the center of the disc at $z=0 . \psi$ is a section of $L^{1 / 2}, L$ being the canonical line bundle. $L$ is trivial topologically and holomorphically. Trivializing it, the Dirac operator is essentially the $\bar{\partial}$ operator, and the action is

$$
I=\frac{i}{2 \pi} \int d^{2} z \psi \bar{\partial} \psi
$$

One can of course choose a flat metric on $D$ (Fig. 1a), though in constructing a Hilbert space and comparing to a canonical formalism it is natural to think in terms of a metric in which the boundary $S$ of $D$ has zero extrinsic curvature (Fig. 1b). 
(a)

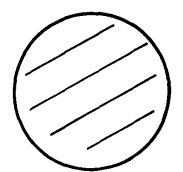

(b)

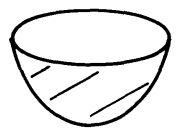

Fig. 1a and b. A disc with flat metric a or embedded with zero extrinsic curvature b

Let us in fact pause to discuss the connection with the canonical formalism, before trying to make contact with Grassmannians. (The following remarks amount to determining which of the two spin structures on a circle is a spin boundary.) In describing the spinor field on $D$ as a function $\psi$ rather than a section of the line bundle $L^{1 / 2}$, we have essentially trivialized the line bundle $L$ by the choice of a one form $d z$. The section of $L^{1 / 2}$ corresponding to $\psi$ is just $\hat{\psi}=\psi \sqrt{d z}$ ( $\sqrt{d z}$ is a formal symbol that transforms like the square root of $d z$ ). For comparing to the canonical formalism, it is not convenient to trivialize $L$ by the one form $d z$ since this is not invariant under rotations of the circle $S$. Introducing Cartesian and polar coordinates by $z=x+i y=r e^{i \theta}$, a section of $L$ near $S$ that is invariant under $\theta \rightarrow \theta+$ constant is, e.g. $d r+i r d \theta$. This choice, though not holomorphic (and illdefined at $\theta=0$ ), is well adapted to comparing with the canonical formalism. The relation between them is $d z=e^{i \theta}(d r+i r d \theta)$. Thus, our section of $L^{1 / 2}$ is $\hat{\psi}=\psi \sqrt{d z}$ $=\psi e^{i \theta / 2} \sqrt{(d r+i r d \theta)}$. Thus, the fermion field $\psi^{\prime}$ of the canonical formalism is really

$$
\psi^{\prime}=\psi e^{i \theta / 2} \text {. }
$$

Since $\psi$ (which extends over $D$ ) is periodic under $\theta \rightarrow \theta+2 \pi$, the factor $e^{i \theta / 2}$ in (3) means that $\psi^{\prime}$ is antiperiodic. Thus, free fermions on $D$ correspond in the canonical formalism to the Neveu-Schwarz sector (antiperiodic boundary conditions) on $S=\partial D$. The spin structure of the Neveu-Schwarz sector is the one that is a spin boundary.

Let $H$ be the space of fermion wavefunctions on the circle, i.e., the space of smooth sections of $L^{1 / 2}$ restricted to the boundary of the circle. On the vector space $H$, we can define the following structures. First, since the product of two spinors is a differential, which can be integrated along a curve in an invariant way, we have the natural bilinear form

$$
\Phi\left(\psi_{1}, \psi_{2}\right)=\oint_{S} \psi_{1} \psi_{2}(\theta) d \theta
$$

Second, on the disc $D$ there is no natural notion of taking the complex conjugate of a spinor field $\psi$, that is, of a section of $L^{1 / 2}$. But on restriction to $S=\partial D, L^{1 / 2}$ reduces to the complexification of the (Neveu-Schwarz sector) spin bundle, which is naturally real. Thus, on restriction to $S$, there is a natural notion of taking the complex conjugate of a spinor field. This gives us the hermitian inner product,

$$
\left(\psi_{1} \mid \psi_{2}\right)=\oint_{S} \psi_{1}^{*} \psi_{2}
$$

which endows $H$ with the structure of a Hilbert space. This Hilbert space structure was essential in [1], entering, for instance, in the precise definition of the Grassmannian Gr. In our considerations, the Hilbert space structure will play a less extensive role. 
Now let us discuss the quantum theory corresponding to (2). The quantum effective action is in some sense $\operatorname{det} \bar{\partial}$; however, the meaning of that infinite determinant must be clarified. The $\bar{\partial}$ operator on the disc has an infinite number of zero eigenvalues,

$$
\bar{\partial} z^{n}=0, \quad n=0,1,2, \ldots,
$$

unless they are eliminated by some suitable boundary condition. (The normalizable zero eigenvalues are $z^{n}, n \geqq 0$, since our disc is $|z| \leqq 1$.) This is a symptom of the fact that to obtain an elliptic operator on a manifold with boundary, such as $D$, it is necessary to adopt a suitable boundary condition, which will eliminate all but perhaps finitely many zero eigenvalues.

A suitable boundary condition must remove roughly half the components. For a second order operator, such as the Laplace operator $\nabla^{2}$, one can choose a local boundary condition. The most convenient local boundary condition is usually to require that the scalar field or its normal derivative should vanish on $S$. For a first order Dirac operator acting on a field with both chiralities, a local boundary condition is likewise possible. The two component Dirac eigenvalue problem

$$
\left(\begin{array}{ll}
0 & \bar{\partial} \\
\partial & 0
\end{array}\right)\left(\begin{array}{l}
\psi \\
\tilde{\psi}
\end{array}\right)=\lambda\left(\begin{array}{l}
\psi \\
\tilde{\psi}
\end{array}\right)
$$

certainly admits the local boundary conditions $\psi=\tilde{\psi}$ on $S$ - a condition familiar from the bag model and from the theory of open strings. However, for the one component chiral Dirac operator, there is no way to choose a local boundary condition. Instead, as in [11], one must in a generalized sense set "half" the components of the field $\psi$ to zero.

Let $H_{+}$be the subspace of $H$ generated by the boundary values of the functions $z^{-1}, z^{-2}, z^{-3}, \ldots$ And let $H_{-}$be the subspace generated by $1, z, z^{2}, \ldots$ Thus, $H_{-}$ consists precisely of boundary values of zero modes of the Dirac operator. The factor $e^{i \theta / 2}$ in (3) means that in the canonical description, $H_{+}$is spanned by wavefunctions

$$
e^{-i \theta / 2}, \quad e^{-3 i \theta / 2}, \quad e^{-5 i \theta / 2}, \ldots,
$$

while $H_{-}$is spanned by

$$
e^{i \theta / 2}, \quad e^{3 i \theta / 2}, \quad e^{5 i \theta / 2}, \ldots
$$

The functions

$$
V_{n}=z^{n}
$$

clearly form a basis for $H$, and in this basis [remembering the $e^{i \theta / 2}$ in (3)], the quadratic form $\Phi$ is

$$
\Phi\left(V_{n}, V_{m}\right)=2 \pi \delta_{n+m+1} .
$$

A typical boundary condition that gives an elliptic $\bar{\partial}$ operator is to require that $\psi$ restricted to $S$ should lie in $H_{+}$. This removes all of the zero eigenvalues of $\bar{\partial}$, since they have boundary values in $H_{-}$. More generally, we may consider any subspace $W$ of $H$ which is comparable to $H_{+}$in a sense described in [1]. Roughly 
speaking, we wish to require that $W$ differs from $H_{+}$only by a finite dimensional amount. Rather than follow the definitions in [1], let us try to see on physical grounds what the requirements on $W$ should be.

Let $\bar{\partial}_{W}$ be the Dirac operator acting on functions whose boundary values are in $W$. One requirement on $W$ must definitely be that the intersection $W \cap H_{-}$should be finite dimensional, so that $\bar{\partial}_{W}$ only has finitely many zero eigenvalues. In the theory of one component chiral fermions, with boundary conditions corresponding to a choice of $W \in G r$, in addition to ellipticity of $\bar{\partial}_{W}$, one also requires that the operator $\bar{\partial}_{W}$ should be skew symmetric - a property that corresponds to hermiticity of $i \bar{\partial}_{W}$ upon Minkowskian continuation. Thus, we require that

$$
\left\langle\psi_{1} \mid \psi_{2}\right\rangle=\frac{i}{2 \pi} \int_{D} d^{2} z \psi_{1} \bar{\partial} \psi_{2}
$$

should be odd under $\psi_{1} \leftrightarrow \psi_{2}$. In fact, upon integrating by parts, we find

$$
\int_{D} d^{2} z \psi_{1} \bar{\partial} \psi_{2}=-\int_{D} d^{2} z \bar{\partial} \psi_{1} \psi_{2}+\oint_{S} \psi_{1} \psi_{2}
$$

so skew symmetry holds only if $\Phi\left(\psi_{1}, \psi_{2}\right)=0$. Here the only restriction on $\psi_{1}$ and $\psi_{2}$ is that their boundary values lie in $W$. Thus, in the theory of one component chiral fermions, the boundary conditions depend on a subspace $W$ of $H$ with the property that for all $\psi_{1}, \psi_{2} \in W, \Phi\left(\psi_{1}, \psi_{2}\right)=0$. This condition means that $W$ is an "isotropic" subspace of $H$ with respect to the quadratic form $\Phi$.

So far we have learned two conditions on $W$ :

(i) $W \cap H_{-}$is finite dimensional so that $\bar{\partial}_{W}$ only has finitely many zero eigenvalues.

(ii) $W$ is isotropic, so that $\bar{\partial}_{W}$ is skew-symmetric.

A third condition is necessary, and we may guess what it is by thinking about the standard space $W=H_{+}$. A look back to (10) reveals that $H_{+}$is indeed isotropic, but also has the following more delicate property. $H_{+}$is a maximal isotropic subspace of $H$; if one added to $H_{+}$any linear combination of the vectors $1, z, z^{2}, \ldots$ not in $H_{+}$, the enlarged space would no longer be isotropic. With this in mind we are led to guess the following additional condition on $W$ :

(iii) $W$ is a maximal isotropic subspace of $H$.

To understand the need for this third condition, let us determine the conditions on $W$ such that the Dirac propagator will exist. The Dirac propagator $G(z, w)$ should be an inverse of the operator $\frac{1}{2 \pi i} \bar{\partial}$; that is, it should be a solution of the equation

$$
2 \pi i \bar{\partial} G(z, w)=\delta^{2}(z-w) .
$$

The only singularity of $G$ should be a pole at $z=w$ with residue 1 . Moreover, the statement that $\psi$ has boundary values in $W$ should mean the following. Regarded as a function of $w$ for fixed $z$ and restricted to $|w|=1, G(z, w)$ is an element of $H$ which we will call $G_{z}(w)$. The boundary conditions are

$$
G_{z}(w) \in W, \text { for all }|z|<1 .
$$


Let us assess the existence of a Dirac propagator in the special case $W=H_{+}$. In that case, the above conditions are obeyed by the standard propagator

$$
\frac{1}{z-w}=-\sum_{n=0}^{\infty} z^{n} \cdot w^{-(n+1)} \text {. }
$$

This series indeed converges for $|z|<1$ and $w$ on the boundary, and the terms in the series (regarded as functions of $w$ ) are all in $H_{+}$, so (16) obeys (15) if $W=H_{+}$. Notice that all of the basis functions $w^{-1}, w^{-2}, \ldots$ of $H_{+}$appear on the right side of (16), with coefficients $\left(z^{n}\right)$ that are linearly independent as functions of $z$. Thus, although the propagator exist with boundary conditions in $H_{+}$, it would not exist if we required that the boundary values of the propagator should be in some proper subspace of $H_{+}$. The fact that $H_{+}$is a maximal isotropic subspace of $H$ is crucial for the existence of the Dirac propagator.

It is true generally that a propagator obeying (15) only exists if $W$ is a maximal isotropic subspace of $H$. Roughly, for a maximal isotropic subspace $W$ the propagator exists and is unique ${ }^{1}$; but if one replaces a maximal isotropic $W$ with a proper subspace, then the boundary conditions (15) become too restrictive, and the propagator no longer exists.

The space of subspaces of $H$ obeying conditions (i), (ii), and (iii) above is what we will call the isotropic Grassmannian $G r^{\prime}$. It is an analogue for one component fermions of the Grassmannian considered in [1].

If we want to encounter the Grassmannian in a framework a little bit closer to that which was envisaged in [1], we must consider a fermion theory with doubled degrees of freedom,

$$
\mathscr{L}=\frac{i}{\pi} \int d^{2} z \tilde{\psi} \bar{\partial} \psi
$$

What are suitable boundary conditions in (17)? Before answering this question, let us consider the following simple mathematical observation.

For any subspace $W$ of $H$, there is a dual space $\tilde{W}$ defined as follows. $\tilde{W}$ consists of all $\psi \in H$ such that

$$
\Phi(\psi, \chi)=0
$$

whenever $\chi \in W$. For instance, if $W=H_{+}$, then $\tilde{W}=W=H_{+}$. More generally, if $W$ is the subspace of $H$ with basis $z^{-k}, z^{-k-1}, \ldots$, then $\tilde{W}$ is the subspace with basis $z^{k-1}, z^{k-2}, \ldots$. Note that whenever one deletes a basis vector from $W, \tilde{W}$ gains a basis vector, because one condition is removed from (18); and conversely, if $W$ is enlarged, $\tilde{W}$ shrinks.

What boundary conditions on $\psi$ and $\tilde{\psi}$ will make the Dirac operator associated with (17) skew-symmetric? Evidently, if we require with $\left.\psi\right|_{S}$ (i.e., $\psi$ restricted to $S=\partial D$ ) should lie in $W$, then $\left.\tilde{\psi}\right|_{S}$ must lie in $\tilde{W}$. Since the boundary conditions on $\tilde{\psi}$ are in this way determined by those on $\psi$, it is possible (as in [1]) to state the requisite conditions in terms of a single subspace $W \subset H$ rather than the pair $W, \tilde{W}$. Thus, we require that $W$ should obey the following condition:

${ }^{1}$ Except when $\bar{\partial}_{W}$ has zero modes; then the propagator exists and is unique in a space orthogonal to the zero modes 
(i') $W \cap H_{-}$is finite dimensional (so that there are only finitely many $\psi$ zero modes); and its dual space $\tilde{W}$ likewise is such that $\widetilde{W} \cap H_{-}$is finite dimensional (so that there are only finitely many $\tilde{\psi}$ zero modes).

Conditions (ii) and (iii) above need not be restated explicitly; they are automatic if the boundary conditions on $\psi$ and $\tilde{\psi}$ are given by a dual pair $W, \widetilde{W}$, since $W \oplus \tilde{W}$ is automatically maximal isotropic as a subspace of the possible boundary values of the pair $(\psi, \tilde{\psi})$. The space of subspaces $W \subset H$ which obey (i') is what Segal and Wilson call Gr. (The connection with the terminology of [1] is as follows. In [1], $G r$ is defined as the family of subspaces $W \subset H$ such that the orthoprojection $W \rightarrow H_{+}$has finite dimensional kernel and cokernel. Finite dimensionality of the kernel is the statement that $W \cap H_{-}$is finite dimensional, and finite dimensionality of the cokerned is the statement that $\tilde{W} \cap H_{-}$is finite dimensional.)

The index of the Dirac operator $\bar{\partial}_{W}$ is defined as the number of $\psi$ zero modes minus the number of $\tilde{\psi}$ zero modes. Clearly, this is the integer $n=\operatorname{dim}\left(H_{-} \cap W\right)$ $-\operatorname{dim}\left(H_{-} \cap \widetilde{W}\right)$. It is well known that the index is a topological invariant. Therefore, the space $G r$ is not connected; its connected components are labeled by the integer $n . n$ is called the "virtual dimension" of $W$ in [1]. In what follows we will generally restrict ourselves to the $n=0$ component of $G r$.

Of course, with $\psi_{1}=(\tilde{\psi}+\psi) / \sqrt{2}, \psi_{2}=i(\tilde{\psi}-\psi) / \sqrt{2},(17)$ is equivalent to

$$
\mathscr{L}^{\prime}=\frac{i}{2 \pi} \int d^{2} z\left(\psi_{1} \bar{\partial} \psi_{1}+\psi_{2} \bar{\partial} \psi_{2}\right) \text {. }
$$

Allowed boundary conditions for (19) that do not "mix" $\psi_{1}$ and $\psi_{2}$ would correspond to a choice of a point in $G r^{\prime} \times G r^{\prime}$ - that is, one point in $G r^{\prime}$ for $\psi_{1}$ and one for $\psi_{2}$. More general elliptic and skew symmetric boundary conditions for (19) would "mix" $\psi_{1}$ and $\psi_{2}$. The most general boundary conditions would be described as follows. Let $H_{1}$ and $H_{2}$ be the spaces of boundary values for $\psi_{1}$ and $\psi_{2}$; let $\hat{H}=H_{1} \oplus H_{2}$. Then a general elliptic and skew symmetric boundary condition in (19) consists of a subspace $\hat{W} \subset \hat{H}$ which obeys (i), (ii), and (iii) above. Let $\hat{G} r$ be the space of such $\hat{W}$. Inside $\hat{G} r$, the Grassmannian $G r$ as we have defined it (or as studied in [1]) is the subspace of boundary conditions that are invariant under the $U(1)$ symmetry

$$
\psi \rightarrow e^{i \alpha} \psi, \quad \tilde{\psi} \rightarrow e^{-i \alpha} \tilde{\psi} .
$$

$\hat{W}$ being invariant under (20) means it must be of the form $\hat{W}=W \oplus \tilde{W}$, with $W$ and $\widetilde{W}$ being spaces of allowed boundary values for $\psi$ and $\tilde{\psi}$, respectively; and (ii), (iii) amount to saying that $\tilde{W}$ must be the dual of $W$ (it must be contained in the dual of $W$ to obey (ii); and it must be the dual to obey (iii)).

From the symmetry (20) follows a characteristic difference between (17) and the one component fermion theory (2). In (17) one may introduce an arbitrary line bundle $E$ and consider $\psi, \tilde{\psi}$ as sections of $L^{1 / 2} \otimes E$ and $L^{1 / 2} \otimes E^{-1}$, respectively. [This is equivalent to introducing a $U(1)$ gauge field with opposite charges for $\psi$ and $\psi$.] In this way, the Grassmannian $G r$ emerges - as in [1] - as a natural framework for studying a Riemann surface endowed with a line bundle. By 
contrast, in the one component fermion theory, there is no natural way to introduce a line bundle, and so the isotropic Grassmannian $G r^{\prime}$ is a natural framework for studying Riemann surfaces that are not endowed with such a line bundle.

Having obtained elliptic and skew symmetric Dirac operators $\bar{\partial}_{W}$, the next step is to attempt to define their determinants $\operatorname{det} \bar{\partial}_{W}$. In fact, in the two component theory (11) we want $\operatorname{det} \bar{\partial}_{W}$, while in the one component theory (2) we want $\sqrt{\operatorname{det} \bar{\partial}_{W}}$ - the square root can be interpreted as the Pfaffian of the infinite dimensional skew form (antisymmetric bilinear form) $\bar{\partial}_{W}$.

Just as for compact Riemann surfaces, the Dirac determinant will be naturally a section of a holomorphic line bundle rather than a function. I will now briefly sketch how this comes about, at the same time using terminology that is appropriate for "fermion Pfaffians" as opposed to fermion determinants.

For $W \in G r^{\prime}$, let $U_{W}=\Gamma_{W}\left(\mathscr{C}^{1 / 2}\right)$ denote the space of sections of $\mathscr{C}^{1 / 2}$ whose restriction to $S$ lies in $W$. The expression

$$
\left\langle\psi_{1}, \psi_{2}\right\rangle=\frac{i}{2 \pi} \int d^{2} z \psi_{1} \bar{\partial} \psi_{2}
$$

defines a skew symmetric bilinear form on elements $\psi_{1}, \psi_{2} \in U_{W}$. We wish to compute the Pfaffian of this skew form. Before tackling the infinite dimensional situation, let us discuss Pfaffians in finite dimension. Let $U$ be a finite dimensional vector space of even dimension $2 k$. A skew symmetric bilinear form $A$ on $U$ is

$$
\left\langle u_{1}, u_{2}\right\rangle=A_{i j} u_{1}^{i} u_{2}^{j},
$$

where $u_{1}$ and $u_{2}$ are elements of $U$ whose components (in some basis) have been denoted $u_{1}^{i}$ and $u_{2}^{j}$, and where $A_{i j}=-A_{j i}$ are matrix elements of $A$. The "Pfaffian" of $A$ is up to normalization

$$
\operatorname{Pf}(A)=\varepsilon^{i_{1} i_{2} \ldots i_{2 k}} A_{i_{1} i_{2}} A_{i_{3} i_{4}} \ldots A_{i_{2 k-1} i_{2 k}} .
$$

Here $\varepsilon^{i_{1} \ldots i_{2 k}}$ is the completely antisymmetric tensor. However, if $U$ is presented merely as a vector space, there is no natural way to normalize $\varepsilon$, which is essentially a volume form on $U$. The possible choices of $\varepsilon$ form a one dimensional complex vector space which we may denote as $V=\wedge{ }^{2 k} U$, the antisymmetric tensor product of $2 k$ copies of $U$. The Pfaffian of $A$ is not naturally a number but rather an element of $V^{*}$, the dual space of $V$. This is just a fancy way of saying that to define the Pfaffian as a number one must supply an element $\varepsilon$ of $V$ (and the result, being proportional to $\varepsilon$, defines a linear map $\operatorname{Pf}(A): V \rightarrow \mathbb{C}$; here $\mathbb{C}$ denotes complex numbers).

Suppose that $U$ is presented as an $n=2 k$ dimensional subspace of a larger vector space $\hat{U}$. In this case we may consider the family $G r_{n}(\hat{U})$ of $n$ dimensional subspaces of $\hat{U}$. For each $U \in G r_{n}(\hat{U})$, we define the one dimensional vector space $V_{U}=\wedge{ }^{2 k} U$. We will sometimes refer to $\wedge^{2 k} U$ as $\operatorname{det} U$. As $U$ varies, $\operatorname{det} U$ or $V_{U}$ varies holomorphically, giving a line bundle $\mathscr{V}$ on $G r_{n}(\hat{U})$. Let $X$ be some complex submanifold of $G r_{n}(\hat{U})$, and $\left\{U_{\lambda} \mid \lambda \in X\right\}$ the corresponding family of subspaces of $\hat{U}$. If we are given a holomorphically varying family of skew bilinear forms $A_{\lambda}$ on the $U_{\lambda}$, then the Pfaffian $\operatorname{Pf}\left(A_{\lambda}\right)$ is a holomorphic section of $\mathscr{V}$, restricted to $X$. 
This is the finite dimensional situation that we wish to imitate in defining the Pfaffian of the Dirac operator or more precisely of the skew form

$$
\left\langle\psi_{1} \mid \psi_{2}\right\rangle=\frac{i}{2 \pi} \int d^{2} z \psi_{1} \bar{\partial} \psi_{2} .
$$

First of all, we must define the determinant line bundle over the Grassmannian, since the Pfaffian is a section of this line bundle.

Instead of getting into technicalities (proper technical treatments can be found in [1] or, in a somewhat different context, in [9]), I would prefer to illustrate the basic idea of the determinant line bundle in a simple and concrete way. To simplify a few formulas, we will temporarily work with $G r$ instead of $G r^{\prime}$ and fermion determinants rather than Pfaffians.

Let $\alpha, \beta$ be a pair of complex numbers, not both zero, and defined up to multiplication by a non-zero complex scalar; thus the pair $(\alpha, \beta)$ defines a point in the complex projective line $P^{1}$. Let $\lambda=\alpha / \beta$ (i.e., $\lambda$ takes values in $\mathscr{C} \cup \infty$, the finite complex plane $\mathscr{C}$ plus the point at infinity; this is a model for $P^{1}$ ). For every $\lambda$, let $W_{\lambda}$ be the subspace of $H$ with basis consisting of the vectors $z^{-2}, z^{-3}, z^{-4}, \ldots$ plus the one additional vector

$$
\alpha+\beta z^{-1}
$$

Note that at $\alpha=0, W_{\lambda}$ is simply $H_{+}$; for all $\lambda, W_{\lambda}$ obeys the proper conditions to be in $G r$. (I leave it to the reader to figure out what is the dual of $W_{\lambda}$.) So the family $W_{\lambda}$ gives an imbedding of $P^{1}$ in $G r$.

Note that $W_{\lambda} \cap H_{-}$is zero except at the one point $\beta=0$, i.e., $\lambda=\infty$. At $\beta=0, W_{\lambda}$ $\cap H_{-}$is one dimensional, so $\bar{\partial}_{W}$ acting on $\psi$ in (17) has a single zero eigenvalue. Therefore, the Dirac "determinant" should have a simple zero.

Consider on the Riemann sphere $P^{1}$ an arbitrary smooth complex valued function $f(w)$ which only has isolated zeros at points $w_{1}, \ldots, w_{k}$. At each zero one can define a topological invariant, the winding number

$$
n_{r}=\frac{1}{2 \pi i} \int_{C} d w \frac{d}{d w} \ln f
$$

with $C$ being a contour that only surrounds the $r^{\text {th }}$ zero. One has the basic relation

$$
\sum_{r} n_{r}=0
$$

for any complex-valued function (as opposed to a section of a line bundle). We can now see that the Dirac "determinant" cannot possibly be a function, since it violates (27). The determinant, restricted to $P^{1}$, has only a single zero, at $\lambda=\infty$, so the sum in (27) is 1 , rather than 0 . This shows us that the Dirac "determinant" must be a section of a line bundle, rather a function, and indeed we can see what the relevant line bundle must be, when restricted to $P^{1} C G r$. Line bundles over $P^{1}$ are classified (topologically and holomorphically) by a single integer, the "degree." A complex line bundle $L$ over $P^{1}$ is said to have degree $k$ if [with the $n_{r}$ defined as in (26)] the zeros of any continuous complex valued section which only has isolated zeros obey

$$
\sum_{r} n_{r}=k
$$


Thus, from what we have said, the determinant line bundle, restricted to $P^{1}$, must be a line bundle of degree 1 .

In the above, we embedded $P^{1}$ in the Grassmannian $G r$ rather than the isotropic Grassmannian $G r^{\prime}$. The sole reason to do so was to simplify the formulas slightly. An embedding of $P^{1}$ in the isotropic Grassmannian can be obtained by taking $W_{\lambda}$ to consist of $z^{-3}, z^{-4}, \ldots$ as well as

$$
\alpha+\beta z^{-2}, \text { and }-\alpha z+\beta z^{-1} .
$$

This has been constructed to respect the isotropic condition. Notice that with this choice $W \cap H_{-}$is zero dimensional for finite $\lambda$ but $t w o$ dimensional at $\lambda=\infty$. This latter fact is no accident. The isotropic condition permits us to define a skew form $\bar{\partial}_{W}$ with boundary conditions $W$, and the kernel of a skew form has a dimension which can only jump in dimension by a multiple of two ${ }^{2}$ (and so in our situation is always even, at it is zero for $W=H_{+}$). Thus, our embedding of $P^{1}$ in $G r^{\prime}$ is such that the Dirac "determinant" would be a section of a line bundle of degree two; it is this which permits the determinant line bundle to have a square root, and the Pfaffian to exist, when restricted to $G r^{\prime}$.

It turns out that the topology of the Grassmannian is such that holomorphic line bundles over it are uniquely determined (topologically and holomorphically) by their restriction to $P^{1}$, so the determinant line bundle is uniquely determined by the above remarks. Instead of pursuing that line of thought, let us now think of the above example in a slightly different way. We will carry out the discussion in a way which is suited to fermion Pfaffians. The "standard" point $H_{+} \in G r^{\prime}$ has basis $z^{-1}$, $z^{-2}, z^{-3}, \ldots$ In general, another point in $G r^{\prime}$ might have all of these basis vectors $z^{-k}$ perturbed. Let us, however, restrict ourselves to the subspace $G r_{k}^{\prime}$ of $G r^{\prime}$ consisting of subspaces of $H$ which contain

$$
z^{-k-1}, \quad z^{-k-2}, \quad z^{-k-3}, \ldots
$$

(The collection of the $G r_{k}^{\prime}$ is dense in $G r^{\prime}$, so in some respects restriction to $G r_{k}^{\prime}$ of finite but arbitrarily large $k$ is not a big loss.) A point $W \in G r_{k}^{\prime}$ will have $k$ additional basis vectors

$$
U_{i}=\sum_{j \geqq-k} u_{i j} z^{j}, \quad i=1 \ldots k .
$$

In general, the sum in (32) runs from $-k$ to $+\infty$, but let us consider the subspace $G r_{k, m}^{\prime}$ of $G r_{k}^{\prime}$ defined by saying that the $u_{i j}$ are zero for $j>m$. Again, the $G r_{k, m}^{\prime}$ for very large $k, m$, can be taken as an approximation to $G r^{\prime}$.

${ }^{2}$ To see this, note that an antisymmetric bilinear form $A_{i j}$ has the canonical form

$$
A=\left(\begin{array}{ccccc}
0 & n_{1} & 0 & 0 & \ldots \\
-n_{1} & 0 & 0 & 0 & \ldots \\
0 & 0 & 0 & n_{2} & \ldots \\
0 & 0 & -n_{2} & 0 & \ldots \\
\vdots & \vdots & \vdots & \vdots & \vdots
\end{array}\right)
$$

with skew "eigenvalues" $n_{k}$. Clearly, the kernel of $A$ jumps in dimension by two when one of the $n_{k}$ goes to zero 
In principle, we would like to understand what we should mean by the Pfaffian of $\bar{\partial}_{W}$ for arbitrary $W \in G r^{\prime}$, but let us restrict the problem to the case $W \in G r_{k, m}^{\prime}$ for some $k, m$. In this case, the problem of defining the determinant line bundle is really a finite dimensional problem. Thus, let $W_{0}$ be the space spanned by (31), and $W^{\prime}$ the space spanned by (32). We have $W=W_{0} \oplus W^{\prime}$. The determinant line bundle det $W$ should then be in some sense ${ }^{3}$

$$
\operatorname{det} W=\operatorname{det} W_{0} \otimes \operatorname{det} W^{\prime} .
$$

Here det $W_{0}$ is ill-defined, because $W_{0}$ is infinite dimensional, but as $W_{0}$ is constant, we can ignore this factor in (33) and identify $\operatorname{det} W$ with $\operatorname{det} W^{\prime} . W^{\prime}$ is a finite dimensional (in fact, $k$ dimensional) subspace of the finite dimensional vector space $H_{k, m}$ spanned by vectors $z^{r}$ for $m \geqq r \geqq-k$. This is precisely analogous to the finite dimensional situation which we discussed first, so by det $W^{\prime}$ we mean simply

$$
\operatorname{det} W^{\prime}=\wedge{ }^{k} W^{\prime},
$$

i.e., the highest exterior power of the finite dimensional vector space $W^{\prime}$. Equations (33) and (34), with det $W_{0}$ replaced by 1 , serve as a precise definition of a line bundle over $G r_{k, m}^{\prime}$ which we will call the Pfaffian line bundle PFAFF. For large $k, m$, this approximates a definition of a line bundle which in fact is defined over all of $G r^{\prime}$. As is indicated by our introductory comments about Pfaffians in finite dimensions, the Pfaffian of the skew form $\bar{\partial}_{W}$ is a holomorphic section of the dual bundle PFAFF*.

To follow [1,9] somewhat more closely, we should aim to define a determinant line bundle DET over $G r$ rather than a Pfaffian line bundle over $G r^{\prime}$. Restricted to $G r^{\prime} \subset G r$, DET is just PFAFF ${ }^{2}$ (just as the determinant of a skew form is the square of the Pfaffian), but we must define DET over $G r$, not just over $G r^{\prime}$. In doing so, we would mostly prefer to avoid Dirac "determinants." Our point of view is that because of anticommutativity of fermions, the Dirac kinetic energy is naturally a skew bilinear form, not an operator, and the Pfaffian is the natural concept. The effective action derived from (19) we would view as the Pfaffian of the skew form

$$
\bar{\partial}_{W}^{\prime}=\left(\begin{array}{cc}
0 & \bar{\partial}_{\tilde{W}} \\
\bar{\partial}_{W} & 0
\end{array}\right),
$$

in the space of fields $(\psi, \bar{\psi})$, not as the determinant of the "operator" $\bar{\partial}_{W}$. In this spirit, we can define the DET bundle as the PFAFF bundle in a larger space. Thus, given $W \in G r$ and its dual space $\widetilde{W}$, we think of $W \oplus \widetilde{W}$ as a subspace of $H \oplus H$ [the latter being the set of boundary values of $(\psi, \tilde{\psi})]$. Approximating $G r$ by some $G r_{k, m}$ as in the above, we have $W=W_{0}+W^{\prime}, \tilde{W}=W_{0}+\tilde{W}^{\prime}$, and a reasoning just as above leads us to the conclusion that the Pfaffian of $\bar{\partial}_{W}^{\prime}$ is a section of

$$
\mathrm{DET}=\wedge^{k} W^{\prime} \otimes \wedge{ }^{k} \tilde{W}^{\prime} \text {. }
$$

This defines the desired line bundle DET over $G r_{k, m}$, the idea being that the Pfaffian of the Dirac skew form (35) is a section of DET*. Equation (36) of course

\footnotetext{
${ }^{3}$ We are borrowing the following fact from finite dimensions. If $A$ and $B$ are finite dimensional vector spaces, say of dimensions $p, q$, then $\wedge^{p+q}(A \oplus B)=\wedge \wedge^{p} A \otimes \wedge{ }^{q} B$, or in other words $\operatorname{det}(A \oplus B)=\operatorname{det} A \otimes \operatorname{det} B$
} 
will serve as a definition only over the $G r_{k, m}$ for finite $k, m$; to get to the completion $G r$ takes a little bit of analysis, as in $[1,9]$.

Having studied the line bundle of which it is a section, the next goal should be to actually define $\operatorname{Pf}\left(\bar{\partial}_{W}\right)$, the Pfaffian of the Dirac skew form. As $W$ varies, $\operatorname{Pf}\left(\bar{\partial}_{W}\right)$ should be a holomorphic section of the dual of the Pfaffian line bundle, restricted to $G r^{\prime}$. (It should be holomorphic because this is so in the finite dimensional case which we should aspire to imitate, unless this proves impossible.) As in the case of finite dimensional Pfaffians, $P f\left(\bar{\partial}_{W}\right)$ should have a zero of order $r$ whenever $\bar{\partial}_{W}$ has $2 r$ zero "eigenvalues." (Recall that the number of zero "eigenvalues" of a skew bilinear form such as $\bar{\partial}_{W}$ is conserved modulo two under any smooth change of parameters. In the case of $\bar{\partial}_{W}$ this number is always even, as it is zero for $W=H_{+}$.) Actually, in view of the fact that there are no non-constant holomorphic functions on $G r^{\prime}$, the above statement about $\operatorname{Pf}\left(\bar{\partial}_{W}\right)$ characterizes $\operatorname{Pf}\left(\bar{\partial}_{W}\right)$ uniquely, assuming it exists.

To compare to [1], we must consider the doubled skew form $\bar{\partial}_{W}^{\prime}$ of (35). In [1], the tau function $\sigma(W)$ was defined as a holomorphic section of DET* that vanishes precisely when $W \cap H_{-} \neq \phi$ or in other words whenever $\overline{\partial_{W}^{\prime}}$ has a zero eigenvalue. Since this property is also the defining property of $\operatorname{Pf}\left(\bar{\partial}_{W}^{\prime}\right)$, it is clear that $\sigma(W)=P f\left(\bar{\partial}_{W}^{\prime}\right)$. More central in [1] than $\sigma(W)$ is the tau function $\tau(W)$, which is essentially a ratio of $\sigma$ functions, and we will refer to the relation between $\sigma$ and $P f\left(\bar{\partial}_{W}\right)$ as the relation between the Dirac Pfaffian and the tau function.

So far we have only discussed uniqueness of $\operatorname{Pf}\left(\bar{\partial}_{W}\right)$ or $\operatorname{Pf}\left(\bar{\partial}_{W}^{\prime}\right)$. As for existence, one approach is to follow [1] or [9]. Alternatively, a down to earth and "physical" approach to defining $\operatorname{Pf}\left(\bar{\partial}_{W}\right)$ is to calculate its variation with respect to a change in $W$, which could be expressed in terms of Green's functions. To compute $\operatorname{Pf}\left(\bar{\partial}_{W}\right)$, one would pick a path $\gamma$ in $G r^{\prime}$ running from $H_{+}$to $W$ and compute the ratio of $\operatorname{Pf}\left(\bar{\partial}_{W}\right)$ to $P f\left(\bar{\partial}_{H_{+}}\right)$by integrating the logarithmic derivative of $P f\left(\bar{\partial}_{W}\right)$ along this path. Defining $\operatorname{Pf}\left(\bar{\partial}_{H}\right)=1$ one thus obtains a definition of $\operatorname{Pf}\left(\bar{\partial}_{W}\right)$. This will give a path dependent result for $\operatorname{Pf}\left(\bar{\partial}_{W}\right)$, which is why the latter is to be seen as a section of a line bundle rather than a function. The program just suggested would be a counterpart of the standard procedure in physical discussions of chiral determinants on manifolds without boundary. It would be worthwhile to carry out this program for manifolds with boundary, but I will not attempt to do so here. However, after a few digressions, we will give later a fairly precise and concrete definition of $\operatorname{Pf}\left(\bar{\partial}_{W}\right)$ along different lines.

It is useful to first consider certain other questions. $\operatorname{Pf}\left(\bar{\partial}_{W}\right)$ is a very canonical holomorphic section of the dual Pfaffian bundle PFAFF*, and has of course a Feynman path integral interpretation,

$$
P f\left(\bar{\partial}_{W}\right)=\int_{W} \mathscr{D} \psi \exp -\frac{i}{2 \pi} \int d^{2} z \psi \bar{\partial} \psi .
$$

Here the symbol $\int_{W} \mathscr{D} \psi$ refers to a Feynman path integral over sections $\psi$ of $L^{1 / 2}$ whose boundary values lie in $W$. While $P f\left(\bar{\partial}_{W}\right)$ (as defined in (37) or in other ways discussed above and below) is a very canonical holomorphic section of PFAFF*, it is by no means the only one. On the contrary, let $P$ be the center of the disc $D$ and 
let $O(P)$ be an arbitrary local operator at $P \in D .{ }^{4}$ Then

$$
P f_{O}\left(\bar{\partial}_{W}\right)=\int_{W} D \psi\left(\exp -\frac{i}{2 \pi} \int \psi \bar{\partial} \psi\right) \cdot O(P)
$$

is a holomorphic section of PFAFF*. Indeed, the assertion that the path integral depends holomorphically on the boundary conditions and the "anomalies" which make it a section of a line bundle rather than a function only depend on local properties near the boundary, and are unaffected by an operator insertion at the center of the disc.

Thus, there are a vast number of holomorphic sections of PFAFF*. Indeed, by a well known principle of conformal field theory, states in the quantum Hilbert space are in one to one correspondence with local operators that may be inserted at the origin (or any other prescribed point). ${ }^{5}$ Thus the ("second quantized") Hilbert space $\mathscr{H}_{S}$ of fermion states on $S=\partial D$ can be identified with $\Gamma$ (PFAFF*), the space of holomorphic sections of PFAFF*. Actually, this might appear to be only an embedding of $\mathscr{H}_{S}$ in $\Gamma$ (PFAFF*), but according to $[1,12]$, this is an isomorphism; all holomorphic sections of PFAFF* are of the form we have described.

Since this statement may require clarification, perhaps it is wise to return to our study of a finite dimensional vector space $\hat{U}$, say of dimension $N$, with an $n=2 k$ dimensional subspace $U$. Fixing a basis $e_{(j)}, j=1 \ldots N$ of $\hat{U}$, the components of a vector $v \in \hat{U}$ will be denoted as $v^{j}$ :

$$
v=\Sigma v^{j} e_{(j)} .
$$

The space $U$ is determined by giving $n$ linearly independent vectors $v_{(i)} \in U$, $i=1 \ldots n$. As in (40), the $v_{(i)}$ can be described explicitly by their components $v_{(i)}^{j}$ in the basis $e_{(j)}$. The wedge product $v_{(1)} \wedge v_{(2)} \wedge \ldots \wedge v_{(n)}$ is an element of the highest exterior power $\operatorname{det} U=\wedge{ }^{n} U$ that we considered earlier. If expanded out in the basis $e_{(j)}$, this wedge product, which is an $n^{\text {th }}$ rank antisymmetric tensor, has components which we may write as $\left(v_{(1)} \wedge v_{(2)} \wedge \ldots \wedge v_{(n)}\right)^{j_{1} \ldots j_{n}}$. As $U$ varies, these components are what we might naively call "functions of $U$," say

$$
a^{j_{1} \ldots j_{n}}(U)=\left(v_{(1)} \wedge v_{(2)} \wedge \ldots \wedge v_{(n)}\right)^{j_{1} \ldots j_{n}}
$$

Actually - as $U$ varies in $G r_{n}(\hat{U})$ - the $a^{j_{1} \ldots j_{n}}$ are not functions on $G r_{n}(\hat{U})$ in the usual sense, since there is no natural way to normalize the $v_{(i)}$. Rather, they are

${ }^{4}$ Thus, $O(P)$ is a linear combination of expressions

$$
\prod_{k=0}^{\infty}\left(\partial^{(k)} \psi\right)^{\mu_{k}}=\psi(P)^{\mu_{0}}(\partial \psi(P))^{\mu_{1}} \ldots\left(\partial^{(k)} \psi(P)\right)^{\mu_{k}} \ldots
$$

where each $\mu_{k}$ is 0 or 1 and all but finitely many $\mu_{k}$ are 0 . Because of fermi statistics, the general local operator (i.e., polynomial in $\psi$ and its derivation) is a linear combination of such monomials ${ }^{5}$ Explicitly, the correspondence is as follows. The quantum fermion theory has operators $\psi_{m}$, $m \in Z+\frac{1}{2}$, with $\left\{\psi_{m}, \psi_{n}\right\}=\delta_{m+n}$. There is a vacuum vector $|\Omega\rangle$ with $\psi_{m}|\Omega\rangle=0, m>0$. The general vector is a linear combination of $\prod_{k=0}^{\infty}\left(\psi_{-k-1 / 2}\right)^{\mu_{k}}|\Omega\rangle$ with each $\mu_{k}$ equal to 0 or 1 , and all but finitely many $\mu_{k}$ zero. The correspondence between operators and states is $\prod_{k=0}^{\infty}\left(\partial^{(k)} \psi\right)^{\mu_{k}} \sim \prod_{k=0}^{\infty}\left(\psi_{-k-1 / 2}\right)^{\mu_{k}}|\Omega\rangle$ 
sections of $(\operatorname{det} U)^{*}$. It should be stressed that for each choice of the indices $a^{i_{1} \ldots i_{n}}$ in (41) (subject to antisymmetry $a^{i_{1} i_{2} \ldots i_{n}}=-a^{i_{2} i_{1} \ldots i_{n}}$, etc.), the "function" $a^{i_{1} \ldots i_{n}}(U)$ is a holomorphic section of $(\operatorname{det} U)^{*}$. And all sections of $(\operatorname{det} U)^{*}$ are linear combinations of these. The latter is a standard and fundamental assertion about the finite dimensional problem which we will not prove here.

The choice of $n$ indices $j_{1} \ldots j_{n}$ in (41) is very similar to choosing a state in a fermion Fock space. Indeed, consider a system of free fermions, with the one particle states being arbitrary vectors in $\widehat{U}$. A state with $n$ particles present has a wave function which is an $n^{\text {th }}$ rank antisymmetric tensor. For any choice of indices $j_{1} \ldots j_{n}$ there is a very special $n$ particle state $e_{\left(j_{1} \ldots j_{n}\right)}=e_{\left(j_{1}\right)} \wedge \ldots \wedge e_{\left(j_{n}\right)}$ in which the filled one particle states are exactly $e_{\left(j_{1}\right)}, \ldots, e_{\left(j_{n}\right)}$. These states are in one to one correspondence with the sections $a^{j_{1} \ldots j_{n}}(U)$ of $(\operatorname{det} U)^{*}$. The $e_{\left(j_{1} \ldots j_{n}\right)}$ are a basis of the $n$ particle portion of the fermion Fock space, and the $a^{j_{1} \ldots j_{n}}(U)$ are a basis of the space of holomorphic sections of $(\operatorname{det} U)^{*}$. These are really dual to each other in a way we will be more precise about later. This is the basic relation between sections of DET* and quantum fermion states; its infinite dimensional analogue is the relation of $\mathscr{H}_{S}$ to the space of holomorphic sections of the appropriate Pfaffian bundle.

We now return to quantum field theory to make this more explicit. In doing so, it is important to note that in the finite dimensional discussion in the last two paragraphs, we did not impose a condition of "isotropy" on the subspace $U \subset \hat{U}$; indeed, there was no quadratic form in the discussion. To compare the results of the last two paragraphs to quantum field theory we have two options: we can repeat the finite dimensional discussion with an isotropic condition, and then we can compare to the quantum field theory (2) of a single fermion; or we can compare the above results as they stand to the two-component theory (17). We will choose the latter path; thus, we will study holomorphic sections of DET* over $G r$, rather that PFAFF* over $G r^{\prime}$. We will consider only the component of $G r$ in which the Dirac index is zero.

We will use the formula (41) in infinite dimensions to give precise descriptions of holomorphic sections of the bundle DET* over Gr. To be more exact, we will give precise formulas for holomorphic sections of $\mathrm{DET}^{*}$ over the finite dimensional approximations $\mathrm{Gr}_{k, m}$ to $\mathrm{Gr}$ that we considered earlier. The formulas will vary nicely with $k, m$, and in the spirit of this paper we will regard that as sufficient evidence that the formulas make sense on $G r$.

Let $w_{1}, w_{2}, \ldots, \ldots$ be a basis of $W$. Because we will actually work on $G r_{k, m}$ for some $k, m$, we know that $z^{-n} \subset W$ for $n>k$, and we thus choose a basis of $W$ with $w_{n}=z^{-n}$ for $n>k$. We want the "components" of the infinite wedge product

$$
w_{1} \wedge w_{2} \wedge w_{3} \wedge \ldots
$$

To do this, write

$$
w_{k}=\sum_{r} \alpha_{k, r} z^{r}
$$

Let $S$ denote a sequence of integers in descending order $s_{1}>s_{2}>s_{3}>\ldots$ Expand

$$
w_{1} \wedge w_{2} \wedge w_{3} \wedge \ldots=\sum_{S} \alpha_{S}(W) z^{s_{1}} \wedge z^{s_{2}} \wedge z^{s_{3}} \wedge \ldots
$$


Since in $G r_{k, m}, w_{r}=z^{-r}$ for $r>k$, it is clear that $\alpha_{S}(W)$ as defined in (44) is zero unless $s_{r}=-r$ for $r>k$. Likewise, it is clear that $\alpha_{S}(W)$ is zero if $s_{1}>m$, as long as we work in $G r_{k, m}$. On the other hand, these are the only restrictions, so $\alpha_{S}(W)$ defines a holomorphic section of DET* for every choice of $k$ distinct integers $s_{1}>\ldots>s_{k}$ in the range $m \geqq s_{i} \geqq-k$. There is no problem in defining the $\alpha_{S}(W)$ rigorously, since they are finite polynomials in the $\alpha_{k, r}$. Taking $k, m$ larger and larger, the restrictions on $S$ become weaker. In fact, for every descending sequence $S=\left\{s_{r}\right\}, r=1 \ldots \infty$ with $s_{r}=-r$ for sufficiently big $r$, the formula (44) defines a holomorphic section of DET* over $G r_{k, m}$ for sufficiently big $k, m$. The fact that the $G r_{k, m}$ are dense in $G r$ shows that any holomorphic section of DET* over $G r$ is nonzero when restricted to some $G r_{k, m}$, so all holomorphic sections of DET* over $G r$ are of the form (44) provided that (41) gives all of the holomorphic sections of DET* in the finite dimensional problem. As already noted, the latter statement is a standard, true statement which we will not prove here.

Let us now interpret our results "physically." We have alleged that the quantum field theory Hilbert space $\mathscr{H}_{S}$ is the space of holomorphic sections of DET* over $G r$. What, indeed, is $\mathscr{H}_{S}$ ? The standard basis in $\mathscr{H}_{S}$ consists of states that are described by saying which one particle levels are filled and which are empty. Let us work in a basis in which the one particle levels are the $z^{n}, n \in Z$. If we agree that at most finitely many states are to be removed from the Dirac sea, the occupied states must be exactly $z^{s_{n}}, n=1,2, \ldots$ where the $s_{n}$, which we may normalize so $s_{1}>s_{2}>\ldots$, are any descending sequence of integers with $s_{r}=-r$ for large enough $r$. This precisely agrees with the counting of holomorphic sections of DET*, confirming the correspondence between states and sections of DET*.

We return now to finite dimensions. In certain respects, the above description is not the most intrinsic. To give as in (40) the components of a vector $v$, one needs a basis $e_{(1)}, \ldots, e_{(N)}$ of $\hat{U}$. These components were used later in (41). To proceed without ever choosing a basis of $\hat{U}$, let $V \in G r_{N-n}(\hat{U})$ be an $N-n$ dimensional subspace of $\hat{U}$. Let $\omega \in \wedge^{N-n} V$ be a volume form for $V$ (i.e., $\omega=f_{(1)} \wedge \ldots \wedge f_{(N-n)}$ with $f_{(j)}$ a basis for $V$ ). Then

$$
\phi_{V}(U)=v_{(1)} \wedge \ldots \wedge v_{(n)} \wedge \omega
$$

is a volume form on $\hat{U} \cdot\left(\phi_{V}(U)\right.$ depends on $\omega$ as well as $V$, but we suppress this in the notation.) If $\alpha$ is a fixed volume form on $\hat{U}$, then

$$
\sigma_{V}(U)=\alpha^{-1} \phi_{V}(U)
$$

is, as $U$ varies, a section of $(\operatorname{det} U)^{*}$. The point of describing things in this way is to make it clear that - without picking a basis or assuming a Hilbert space structure for $\hat{U}-$ it is in the most canonical way a "complementary subspace" to $U$, that is a space $V \subset \hat{U}$ of dimension $N-n$, that defines a section of $(\operatorname{det} U)^{*}$. Instead of saying that one gets a holomorphic section of $\operatorname{det} U^{*}$ for every "set of indices" $i_{1}, \ldots, i_{n}$, it is much better to say that one gets such a section for every $N-n$ dimensional subspace $V \subset \hat{U}$. This is much better not just because the indices are suppressed but more importantly because in this form the statement is true without a choice of basis for $\hat{U}$; it is indeed the $N-n$ dimensional subspace $V$, no more and no less, which is needed to define a holomorphic section of $\operatorname{det} U^{*}$. We could well suppress 


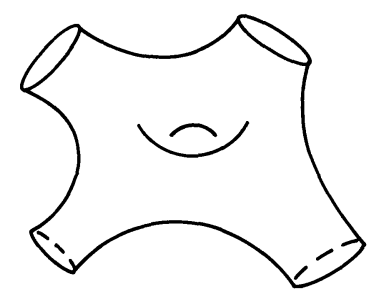

Fig. 2. A Riemann surface with $n$ boundary components

the basis $v_{(i)}$ of $V$; if $\eta$ is a volume form for $V$, our section is

$$
\sigma_{V}(U)=\alpha^{-1} \eta \wedge \omega \text {. }
$$

The symmetry of this formula in $\eta$ and $\omega$ (or $U$ and $V$ ) makes it clear that what we have is really a section of $(\operatorname{det} U)^{*} \otimes(\operatorname{det} V)^{*}\left(\right.$ or of $(\operatorname{det} U)^{*} \otimes(\operatorname{det} V)^{*} \otimes \operatorname{det} \hat{U}$, in view of the dependence on $\alpha$ ). It should be stressed that in this way of expressing things, we never require a Hilbert space structure on $\hat{U}$.

A section of $(\operatorname{det} U)^{*}$ which is of the form $\sigma_{V}(U)$ for some $V$ will be called a "primitive section" in what follows. A primitive section is one which is described by giving a list of one particle states (i.e. a basis of $V$ ). It is not the case that every section of $(\operatorname{det} U)^{*}$ is a primitive section. Rather, the general section of $(\operatorname{det} U)^{*}$ can be expanded as a linear combination of primitive sections:

$$
\phi(U)=\sum_{i} \lambda_{i} \cdot \sigma_{V_{i}}(U)
$$

where $\lambda_{i}$ are complex numbers and $V_{i}$ are some points in $G r_{N-n}(\hat{U})$. The expansion in (48) is far from being unique. It becomes unique if one fixes a basis $e_{(1)}, \ldots, e_{(N)}$ of $\hat{U}$, as in our initial discussion, and considers only spaces $V_{j_{1} \ldots j_{N-n}}$ spanned by subsets $e_{\left(j_{1}\right)}, \ldots, e_{\left(j_{N}-n\right)}$. This is essentially what we did (in infinite dimensions) in writing (44).

In this description, a Hilbert space structure of $\hat{U}$ was never required. To specify a section of $(\operatorname{det} U)^{*}$, with $\operatorname{dim} U=n, \operatorname{dim} \hat{U}=N$, required a choice of an $N-n$ dimensional subspace $V \subset \widehat{U}$. If, however, $\hat{U}$ has a Hilbert space structure, the choice of the $N-n$ dimensional subspace $V$ is equivalent to a choice of an $n$ dimensional subspace $V_{\perp}$ - its orthogonal complement. In our preliminary discussion with a chosen fixed basis $e_{(1)}, \ldots, e_{(N)}$ of $\hat{U}$, a natural Hilbert space structure was present implicitly - namely the one defined by $\left\langle e_{(j)} \mid e_{(i)}\right\rangle=\delta_{j i}$.

We now return to the infinite dimensional situation. We would like to describe the particular holomorphic sections of DET* that arise naturally in certain "physical" situations.

Consider as in Fig. 2 a Riemann surface $\Sigma$ with $n$ boundary components, namely circles $S_{1}, S_{2}, \ldots, S_{n}$. Fixing on $\Sigma$ a square root $L^{1 / 2}$ of the canonical line bundle, we wish to study the chiral Dirac operator $\bar{D} .{ }^{6}$ (As there is no convenient trivialization of $L^{1 / 2}$ on $\Sigma$, we refer to the Dirac operator as $\bar{D}$, and not $\bar{\partial}$ as in our

\footnotetext{
${ }^{6}$ We will study one component chiral fermions. In the two component case, we would be free to twist $L^{1 / 2}$ by a line bundle
} 


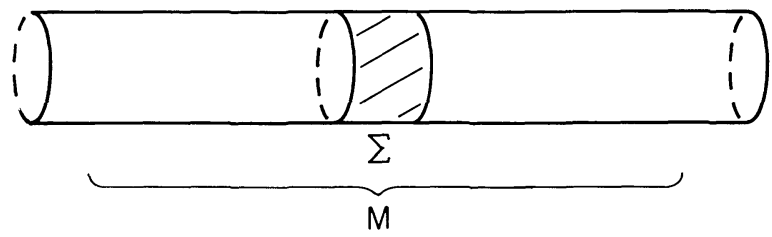

Fig. 3. A finite cylinder $\Sigma$ embedded in an infinite one $M$

study of the Dirac operator on the disc.) On each $S_{k}$, there is a Hilbert space $H_{k}$ of possible one particle states, a Grassmannian $G r_{k}^{\prime}$ of possible boundary conditions, and a determinant line bundle $\mathrm{DET}_{k}$ over $G r_{k}^{\prime}$. A "good" Dirac operator requires the choice of an element $W_{1} \times W_{2} \times \ldots \times W_{k}$ of $G r_{1}^{\prime} \times G r_{2}^{\prime} \times \ldots \times G r_{k}^{\prime}$. The fermion Pfaffian $\operatorname{Pf}\left(\bar{D}_{\left(W_{1}, W_{2}, \ldots, W_{k}\right)}\right)$ is then a holomorphic section of $\mathrm{DET}_{1}^{*} \otimes \ldots \otimes \mathrm{DET}_{k}^{*}$.

The fact that one obtains in this way a holomorphic section of the tensor product $\otimes_{k} \mathrm{DET}_{k}^{*}$ is analogous to the fact that in finite dimensions (46) is really a section of $(\operatorname{det} U)^{*} \otimes(\operatorname{det} V)^{*}$. The subspaces $W_{k} \subset H_{k}$ are complementary in a sense somewhat analogous to the role of $U$ and $V$ in our finite dimensional problem. The analogy perhaps can be sharpened slightly by considering a Riemann surface $\Sigma$ which is a sphere with two holes cut out. Let $M$ be the cylinder $0 \leqq \phi \leqq 2 \pi$, $-\infty<t<\infty$. $\Sigma$ can always be embedded in $M$ as the segment $0 \leqq t \leqq T$ for some $T>0$ (Fig. 3). On $\Sigma, L^{1 / 2}$ has a canonical trivialization and $\bar{D}$ reduces to $\frac{\partial}{\partial \bar{z}}$, $z=t+i \phi$. If we do not worry about boundary conditions, the $\bar{D}$ operator on $\Sigma$ has an infinite dimensional kernel. If continued from $\Sigma$ to $M$, the zero eigenfunctions blow up for $t>T$ or for $t<0$ (i.e. they have singularities either at finite $t$ or for $t$ $\rightarrow \pm \infty$ ). Boundary conditions corresponding to the standard point $H_{+(1)}$ in $G r_{1}^{\prime}$ would remove the wave functions that are singular for $t \rightarrow-\infty$, while a similar choice of $H_{+(2)}$ in $G r_{2}^{\prime}$ would remove the wave functions that are singular for $t \rightarrow+\infty$. Together, these remove the kernel of $\bar{D}$ on $\Sigma$. More generally, the choice of general points $W_{1}$ and $W_{2}$ in $G r_{1}^{\prime}$ and $G r_{2}^{\prime}$ removes all but perhaps finitely many zero modes of $\bar{D}_{\left(W_{1}, W_{2}\right)}$. $W_{1}$ and $W_{2}$ remove in a sense complementary parts of the kernel of $\bar{D}$. The analogy with our finite dimensional situation is perhaps closest if one considers the intersection $\operatorname{ker} \bar{D} \cap W_{1}$ to play the role of $U$, and the intersection $\operatorname{ker} \bar{D} \cap W_{2}$ to play the role of $V$. By $\operatorname{ker} \bar{D} \cap W_{i}$, for $i=1,2$, we mean the subspace of $\operatorname{ker} \bar{D}$ consisting of wavefunctions whose restriction to $S_{i}$ is in $W_{i}$.

Let $T$ be the thickness of $\Sigma$. For any $T$, the formula

$$
\phi^{T}\left(W_{1}, W_{2}\right)=\operatorname{Pf}\left(\bar{D}_{\left(W_{1}, W_{2}\right)}^{T}\right)
$$

(we denote the Dirac operator as $\bar{D}^{T}$ to stress its dependence on $T$ ) gives a section of $\mathrm{DET}_{1}^{*} \otimes \mathrm{DET}_{2}^{*}$. Before attempting to compare to notions of Segal and Wilson we must note the following. $H_{+(1)} \cap \operatorname{ker} \bar{D}$ and $H_{+(2)} \cap \operatorname{ker} \bar{D}$ (that is, the subspaces of ker $\bar{D}$ with boundary values in $H_{+(1)}$ or $H_{+(2)}$ ) are not comparable spaces, ${ }^{7}$ since one consists of wave functions that decay as $t \rightarrow+\infty$ and one consists of wave functions that decay as $t \rightarrow-\infty$. However, if we recall the natural Hilbert space structure of, say, $H_{(2)}$, then $H_{+(2)}$ has an orthogonal complement $H_{+(2)}^{\perp}$ in $H_{(2)}$.

\footnotetext{
${ }^{7}$ That is, they do not in any sense differ by a finite dimensional amount
} 


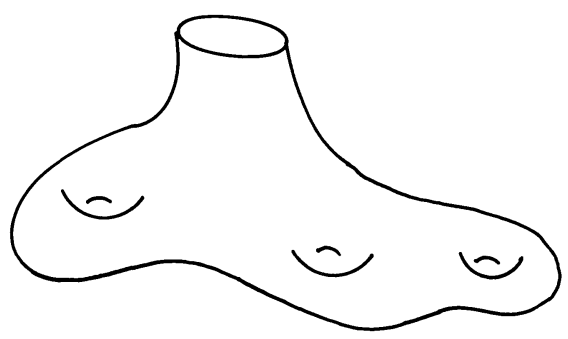

Fig. 4. A Riemann surface with one boundary component and arbitrary genus

Taking the orthogonal complement tends to reverse the direction in which the functions grow, and indeed $H_{+(2)}^{\perp} \cap \operatorname{ker} \bar{D}$ is comparable to $H_{+(1)} \cap \operatorname{ker} \bar{D}$. Likewise $W_{1} \cap \operatorname{ker} \bar{D}$ and $W_{2} \cap \operatorname{ker} \bar{D}$ are not comparable, but $W_{1} \cap \operatorname{ker} \bar{D}$ and $W_{2}^{\perp} \cap \operatorname{ker} \bar{D}$ are. For $T \rightarrow 0, W_{1} \cap \operatorname{ker} \bar{D}$ and $W_{2}^{\perp} \cap \operatorname{ker} \bar{D}$ reduce to $W_{1}$ and $W_{2}^{\perp}$, and $\phi^{T}\left(W_{1}, W_{2}\right)$ reduces to what Segal and Wilson would call the determinant of the orthoprojection $\lambda: W_{1} \rightarrow W_{2}^{\perp}$ from $W_{1}$ to $W_{2}^{\perp}$. Indeed $\operatorname{det} \lambda=0$ precisely if

$$
W_{1} \cap W_{2} \neq(\phi),
$$

while $\phi^{T}\left(W_{1}, W_{2}\right)=0$ precisely if

$$
W_{1} \cap W_{2} \cap \operatorname{ker} \bar{D} \neq(\phi),
$$

since under this condition there is a Dirac zero eigenvalue which is not removed by boundary conditions at either end. Clearly (51) reduces to (50) as $T \rightarrow 0$. As det $\lambda$ and $\lim _{T \rightarrow 0} \phi^{T}\left(W_{1}, W_{2}\right)$ have the same zeros, they must coincide (up to normalization).

We are finally ready to consider a situation that is closer to the essence of the ideas in [1]. Consider a Riemann surface $\Sigma$ (Fig. 4) with one boundary component $S$ and arbitrary genus. We will consider the case of one component chiral fermions. The Dirac operator $\bar{D}_{W}$ depends on the choice of a point $W \in G r^{\prime}$. Its Pfaffian $\psi(W)$ $=P f\left(\bar{D}_{W}\right)$ is a holomorphic section of $\mathrm{PFAFF}^{*}$ and thus is a state in the second quantized Hilbert space $\mathscr{H}_{S}$ associated with the free fermion quantum field theory on $S$.

These facts are a somewhat exotic manifestation of some general principles of quantum field theory. For any quantum field theory, not necessarily conformally invariant, the process of "integrating out" the surface $\Sigma$ will produce a state in the Hilbert space $\mathscr{H}_{S}$ associated with the boundary $S$. This always arises as follows. States $\psi \in \mathscr{H}_{S}$ are always functionals of some kind on the boundary conditions that arise in the Feynman path integral. Performing the Feynman path integral on $\Sigma$ with varying boundary conditions on $S$, one gets a functional of the boundary values and thus a vector in $\mathscr{H}_{S}$.

The detailed realization of this general principle depends on the theory considered. In the relatively simple case of a real (non-chiral) boson, the Feynman path integral involves integration over real valued functions $X: \Sigma \rightarrow R$ ( $R$ denotes the real numbers). In performing the integral, one can fix a function $Y: S \rightarrow R(S=\partial \Sigma)$, and integrate only over functions $X$ whose restriction to $S$ is $Y$. 
This gives a functional of $Y$,

$$
\Psi(Y)=\int_{\left.X\right|_{S}=Y} \mathscr{D} X e^{-\frac{1}{2} \int(\nabla X)^{2}}
$$

(The notation $\left.X\right|_{S}=Y$ refers to integration over maps $\Sigma \rightarrow R$ that equal $Y$ on $S$.) As $Y$ varies, $\Psi(Y)$ is a functional on real valued functions on $S$ or in other words a state in the second quantized Hilbert space of free bosons on $S$.

For chiral fermions, the choice of boundary conditions is a more exotic choice of a point $W \in G r^{\prime}$. The Pfaffian $P f\left(\bar{D}_{W}\right)$ describes the dependence of the Feynman path integral on the boundary values. As such it is a fit counterpart to the more obvious object $\Psi(Y)$ which arises in the theory of real bosons.

Now we would like to identify the particular state $\operatorname{Pf}\left(\bar{D}_{W}\right)$ that arises for chiral fermions by "integrating out $\Sigma$." We will show that it is a "primitive state" in the sense described earlier; that is, it can be described by saying which single particle levels are filled and which are empty. (Recall that the general holomorphic section of PFAFF* is not a primitive state in that sense, but a linear combination of primitive states.) And we will see that the particular primitive state that arises is the one studied in great detail by Segal and Wilson.

In the chiral fermion theory of Eq. (2), $\psi$ is an operator valued section of $L^{1 / 2}$. Its classical field equation is

$$
\bar{D} \psi=0 \text {. }
$$

This equation can be used inside the Feynman path integral

$$
Z=\int_{W} \mathscr{D} \psi \exp -\frac{i}{2 \pi} \int \psi \bar{D} \psi,
$$

as long as there are no insertions of operators that do not commute with $\psi$. The argument for this is standard. ${ }^{8}$ One makes the affine change of variables $\psi \rightarrow \psi+\varepsilon$, which leaves the measure $\mathscr{D} \psi$ invariant. (One requires $\left.\varepsilon\right|_{S}$ to be in $W$ so that this transformation preserves the boundary conditions.) To first order in $\varepsilon$, the change in action is $\frac{2 i}{\pi} \int \varepsilon \bar{D} \psi$, and the invariance of $Z$ under the change of integration variables gives

$$
0=\int \mathscr{D} \psi \exp -\frac{i}{2 \pi} \int \psi \bar{D}_{W} \psi \cdot \int \varepsilon \bar{D} \psi
$$

The assertion that this is true for arbitrary $\varepsilon$ is the statement $\bar{D} \psi=0$. The argument would not hold, of course, for a more general path integral

$$
\int \mathscr{D} \psi \exp -\frac{i}{2 \pi} \int \psi \bar{D}_{W} \psi \cdot \prod_{i} O_{i}\left(P_{i}\right)
$$

with operators $\prod_{i} O_{i}\left(P_{i}\right)$ that are not invariant under $\psi \rightarrow \psi+\varepsilon$. We will discuss the resulting modifications in Sect. (3).

Let $f$ be a $c$-number section of $L^{1 / 2}$ which is holomorphic, i.e. $\bar{D} f=0$. Then $f \psi$ is an operator-valued section of $L$, i.e. a differential form. In fact, $f \psi$ is

\footnotetext{
${ }^{8}$ It has been given independently in this context in [6]
} 
holomorphic in the naive sense, i.e. $\bar{\partial}(f \psi)=0$. (On differential forms $\bar{D}$ reduces to $\bar{\partial}$.) Thus

$$
0=\int_{W} \mathscr{D} \psi \exp -\frac{i}{2 \pi} \int \psi \bar{D} \psi \cdot \int_{\Sigma} \bar{\partial}(f \psi)
$$

Integrating by parts, we have $\int_{\Sigma} \bar{\partial}(f \psi)=\oint_{S} f \psi$, so

$$
0=\int_{W} \mathscr{D} \psi \exp -\frac{i}{2 \pi} \int \psi \bar{D} \psi \cdot \oint_{S} f \psi \text {. }
$$

Now we must recall some basic ideas about Feynman path integrals. The Feynman path integral $\int_{W} \mathscr{D} \psi \exp -I$ constructs a state $\left|\Omega_{\Sigma}\right\rangle$ in $\mathscr{H}_{S}$. If $\hat{O}$ is an operator on $\mathscr{H}_{S}$ and one wishes to compute $\hat{O} \Psi$, this is done in path integrals by computing $\int_{W} \mathscr{D} \psi(\exp -I) \cdot O$. The right-hand side of Eq. (58) is precisely of this form, with $O=\oint_{S} f \psi$, so we read off that

$$
0=\oint_{S} f \psi \cdot\left|\Omega_{\Sigma}\right\rangle
$$

Thus, the state $\left|\Omega_{\Sigma}\right\rangle$ is annihilated by $\oint_{S} f \psi$ whenever $f$ can be extended to a holomorphic section of $L^{1 / 2}$ on $\Sigma$. Thus the state $\left|\Omega_{\Sigma}\right\rangle$ can be characterized (uniquely, as we will argue) by saying that the one particle states which are boundary values of holomorphic sections of $L^{1 / 2}$ are the filled states.

This state is thus the primitive state associated with a very particular point $W_{\Sigma}$ on the Grassmannian: $W_{\Sigma}$ is spanned by sections $f$ of $\left.L^{1 / 2}\right|_{S}$ that extend holomorphically over $\Sigma$.

To check that this makes sense, we must (as we are studying here one component chiral fermions) verify that the point $W_{\Sigma}$ so obtained is an isotropic point on the Grassmannian. Indeed if $f$ and $g$ are holomorphic sections of $L^{1 / 2}$ over $\Sigma$, then

$$
0=\int_{\Sigma} \bar{\partial}(f g)=\oint_{S} f g
$$

This is the assertion that $W_{\Sigma}$ is isotropic. That $W_{\Sigma}$ is maximal isotropic will be verified presently.

$W_{\Sigma}$ is essentially the point on $G r$ that Segal and Wilson associate with the Riemann surface $\Sigma$. However, they are in effect considering two component chiral fermions. Their $\psi$ is a section of $L^{1 / 2} \otimes E$ for some line bundle $E$. As a result $f$ is a section of $L^{1 / 2} \otimes E^{-1}$. Although (59) still goes through, showing that $\left|\Omega_{\Sigma}\right\rangle$ is the primitive state associated with a point $W_{\Sigma} \in G r,(60)$ has no analogue. [Indeed, $f g$ is a section of $L \otimes E^{-2}$ rather than a differential form, so $\bar{D}(f g)$ cannot be integrated over $\Sigma$ in an invariant way.]

The fact that (59) uniquely characterizes the state $\left|\Omega_{\Sigma}\right\rangle$ depends on the following. Let $\psi_{f}=\oint f \psi$. The canonical anticommutators are $\left\{\psi_{f}, \psi_{g}\right\}=\oint f g$. The $\psi_{f}$ for $f \in W_{\Sigma}$ thus anticommute with each other. They form in fact a maximal isotropic (or anticommuting) subalgebra of the Clifford algebra; for $g$ not in $W_{\Sigma}$, there is $f$ in $W_{\Sigma}$ with $\left\{\psi_{f}, \psi_{g}\right\}=\oint f g \neq 0$. Indeed, if $g$ is the boundary value of a 
section of $L^{1 / 2}$ that is meromorphic rather than holomorphic, ${ }^{9}$ then (38) is replaced by $\oint_{S} f g=2 \pi i \sum_{P} \operatorname{Res}_{P}(f g)$, where $P$ runs over poles of $g$ and $\operatorname{Res}_{P}$ denotes the residue at $P$; given $g$, one can pick a holomorphic $f$ to make this nonzero. The Hilbert space $H_{S}$ is an irreducible representation of the Clifford algebra of the $\psi_{f}$. In an irreducible representation of a Clifford algebra, a vector $\left(\left|\Omega_{\Sigma}\right\rangle\right.$ in our case) annihilated by a maximal isotropic subalgebra is unique. The argument we have just sketched showing that the $\psi_{f}, f \in W_{\Sigma}$ are a maximal isotropic subalgebra of the Clifford algebra shows at the same time that $W_{\Sigma}$ is a maximal isotropic subspace of $H$, so we have verified that it obeys the conditions (i), (ii), (iii) above.

To recapitulate this discussion, it follows from general principles of quantum field theory that integrating out $\Sigma$ will give an element of the Hilbert space $H_{S}$. What is striking about free fermion field theory is that there are special states, which we have called primitive states, which in some sense are particularly simple. These are the states associated with points in the nonlinear space $G r^{\prime} .{ }^{10}$ Integrating out $\Sigma$ always gives one of these special states - it maps Riemann surfaces with boundary into $G r^{\prime}$, not just into $\mathscr{H}_{S}$. One may ask whether there is in general conformal quantum field theory - and not just in the theory of free fermions - an analogue of the geometrically interesting nonlinear space $G r^{\prime}$.

In a weak sense, one may argue that there is such an analogue at least for holomorphic conformal field theories. For simplicity, let us consider only the primary fields $\phi_{i}, i=1 \ldots N$ of a conformal field theory. (Descendants could be included, however, by treating observables as sections of a vector bundle that is not just a sum of line bundles.) Let $S_{i}$ be the spin of $\phi_{i}$. Thus $\phi_{i}$ is an operator valued section of $L^{S_{2}}$. Let $f$ be a $c$-number holomorphic section of $L^{1-S_{i}}$ on $\Sigma$, so that $f \phi_{i}$ is a holomorphic operator valued differential form. The argument leading to (59) then shows that

$$
\oint_{S} f \phi_{i}\left|\Omega_{\Sigma}\right\rangle=0 .
$$

Presumably, $\left|\Omega_{\Sigma}\right\rangle$ is completely characterized (up to normalization) by this equation. Thus, in holomorphic conformal field theory the state $\left|\Omega_{\Sigma}\right\rangle$ can presumably always be described by specifying which moments of conformal fields annihilate it. (It is necessary in general to use descendants as well as conformal fields.) The space of states which are primitive in this sense may have an interesting geometrical structure in general and not just for free fermions. However, in the case of free fermions it is possible to give an economical description by focussing on the elementary field $\psi$ only; for a general holomorphic conformal field theory there is no clear analogue of this.

\section{Multiplicative Ward Identities}

Symmetries in quantum field theory are usually studied by means of Ward identities. When we think of Ward identities, we usually think of infinitesimal

\footnotetext{
${ }^{9}$ It is enough to suppose that $g$ is the boundary value of a meromorphic function, since every smooth function on the circle can be approximated by such

${ }^{10}$ Other states are linear combinations of primitive states
} 
transformations - and thus we usually work at the Lie algebra level. It can happen, though, that a group of interest does not correspond to any Lie algebra. An obvious case of a group with no Lie algebra is a finite group. A subtler example of a group that does not correspond to any Lie algebra is the multiplicative group $G$ of global non-zero meromorphic functions on a Riemann surface. There is no Lie algebra for this group, since a global meromorphic function which does have poles or zeros, say $f(z) \sim\left(z-z_{0}\right)^{n}$, for $z \rightarrow z_{0}$, is not the exponential of a single-valued function. In this section, we will formulate Ward identities associated with the group G. Of necessity, they will be "multiplicative Ward identities," relevant to a group action, as opposed to "additive Ward identities," associated with the action of a Lie algebra.

There are several motivations for developing the notion of multiplicative Ward identities. First, such identities, as we will see, can illuminate some relations described in [1]. Second, multiplicative Ward identities shed a new light on bosonization of fermions, a subject which recently has been developed in a global context [13]. And third, multiplicative Ward identities are a crucial ingredient in the connection we will describe in Sects. four and five between current algebra on Riemann surfaces and the modern theory of automorphic forms. The identities we will develop are similar in spirit to one of the techniques used by D'Hoker and Giddings in their work on the relation between the light cone and covariant formulations of string theory [21].

We shall study, on a Riemann surface $\Sigma$ which may have boundary, the two component chiral fermion theory described by the Lagrangian

$$
\mathscr{L}=\frac{i}{\pi} \int_{\Sigma} \tilde{\psi} \bar{D} \psi
$$

$\psi$ and $\tilde{\psi}$ are operator-valued sections of a square root $L^{1 / 2}$ of the canonical line bundle $L$. In what follows we will discuss certain aspects of the dependence of the fermion determinant on boundary conditions defined by a point $W \in G r$. We will discuss the determinant simply as a holomorphic section of DET*, without worrying about the metric on DET*; from this point of view the conformal anomaly is immaterial and we will ignore it below. If one is uncomfortable with this point of view, one can imagine that we are really discussing ratios like $P f\left(\bar{D}_{W}^{\prime}\right) / P f\left(\bar{D}_{H_{+}}^{\prime}\right)\left(H_{+}\right.$is the standard point in the Grassmannian discussed in the last section); such ratios are free of conformal anomaly. Adopting one or the other of these viewpoints, we may proceed in what follows as if there is no conformal anomaly.

Let $f$ be a global non-zero holomorphic function on $\Sigma-$ so $[\bar{D}, f]=0$ and $f$ has neither zeros nor poles. Then (62) is formally invariant under

$$
\psi \rightarrow \psi^{\prime}=f \psi, \quad \tilde{\psi} \rightarrow \tilde{\psi}^{\prime}=f^{-1} \tilde{\psi} .
$$

Of course for such a non-constant global function $f$ to exist, $\Sigma$ must have a boundary. For notational simplicity, we suppose the boundary to consist of a single circle $S$. In this case, though the action (62) is invariant under $f$, the boundary conditions are not. The boundary conditions are that $\psi$ must lie in a subspace $W$ of the space $H$ of possible boundary values (and $\tilde{\psi}$ must lie in the dual space $\bar{W}$, as described in the last section). Let the functions $\left\{u_{1}, u_{2}, \ldots\right\}$, be a basis for $W$. If $\psi$ lies 

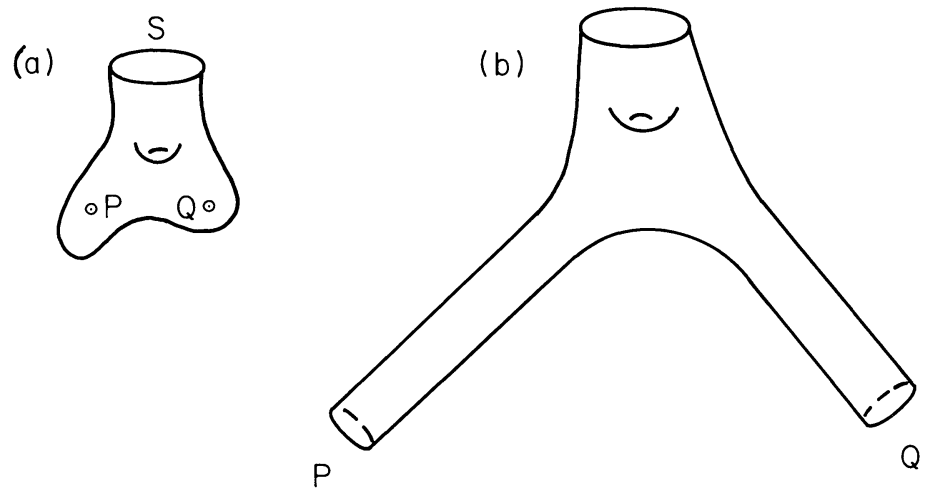

Fig. 5a and b. A Riemann surface $\Sigma$ with boundary $S$ and points $P_{i}$ and $Q_{j}$ a. Upon deleting those points, a conformal rescaling that "projects them to infinity" can restore completeness in the metric sense $\mathbf{b}$

in the space $W$ spanned by $\left\{u_{1}, u_{2}, \ldots\right\}$, then clearly $\psi^{\prime}=f \psi$ will lie in the space spanned by $\left\{f u_{1}, f u_{2}, \ldots\right\}$ - a point in the Grassmannian which we will call $f W$. The transformation $W \rightarrow f W$ is an action of the group $G^{\prime}$ of global invertible holomorphic functions (holomorphic, that is, on the surface-with-boundary $\Sigma$ ) on the Grassmannian. If $\Sigma$ has several boundary components, $G^{\prime}$ acts on the relevant product of Grassmannians. This action of $G^{\prime}$ on the Grassmannian entered in [1] in the special case with $\Sigma$ a disc. There it was shown that $G^{\prime}$ actually acts on the determinant line bundle, and not just on the Grassmannian.

Thus, under the change of variables (63), the action is invariant, but the Dirac operator $\bar{D}_{W}$ is transformed into $\bar{D}_{f W}$. It therefore must be that the Dirac Pfaffian is invariant under the $G$ action,

$$
\hat{f} \cdot \operatorname{Pf}\left(\bar{D}_{W}\right)=P f\left(\bar{D}_{f W}\right) .
$$

(Here $\hat{f}$ denotes the lift of $f$ to act on DET*, and could have been dropped if the Pfaffian were an ordinary function rather than a section of a line bundle.) Equation (64) is our simplest multiplicative Ward identity. As long as $f$ has neither zeros nor poles, there is little more to say.

We now move on to the case where $f$ has zeros and poles - say simple zeros at points $Q_{1} \ldots Q_{l}$ and simple poles at points $P_{1} \ldots P_{k}$. (As long as $\Sigma$ has a boundary, there is no reason to have $l=k$.) Clearly, in this more general context, the transformation (41) is not a symmetry - it is not even a well-defined transformation of the field variables.

To remedy this situation, we will work on the Riemann surface $\Sigma^{\prime}$ which consists of $\Sigma$ with the points $P_{1} \ldots P_{k}$ and $Q_{1} \ldots Q_{l}$ removed. Of course, the surface $\Sigma^{\prime}$ is not "complete." However, conformal invariance makes it possible to treat $\Sigma^{\prime}$ rather like a complete surface at least from the geometric point of view. We simply pick on $\Sigma^{\prime}$ a metric (compatible with the complex structure of $\Sigma^{\prime}$ ) in which the missing points are "infinitely far away," as in Fig. 5b.

According to conformal invariance, the fermion determinant on the compact Riemann $\Sigma$ of Fig. 5a equals the determinant on the metrically complete but not compact surface $\Sigma^{\prime}$ of Fig. $5 \mathrm{~b}$ provided that we specify the right class of functions in 
taking the latter determinant. The right class of functions are simply the square integrable ones,

$$
\int_{\Sigma^{\prime}}|\psi|^{2}<\infty, \quad \int_{\Sigma^{\prime}}|\tilde{\psi}|^{2}<\infty
$$

Now clearly, if we make a change of variables from $\psi$ and $\tilde{\psi}$ to $\psi^{\prime}$ and $\tilde{\psi}^{\prime}$, the class of functions within which we should take the determinant is modified. The new conditions are

$$
\int_{\Sigma^{\prime}}\left|f^{-1} \psi\right|^{2}<\infty, \quad \int_{\Sigma^{\prime}}\left|f \tilde{\psi}^{\prime}\right|^{2}<\infty
$$

In practice, it is near poles and zeros of $f$ that the allowed class of functions $\psi^{\prime}$ and $\tilde{\psi}^{\prime}$ differ from the allowed $\psi$ and $\tilde{\psi}$. We have thus identified the "Ward identity" associated with multiplication by $f$. A fermion determinant with boundary conditions described by $W \in G r$ on the boundary of $\Sigma^{\prime}$ and by the class of functions (65) on the "infinite ends" of $\Sigma^{\prime}$ is equivalent to a determinant with boundary condition determined by $f W$ and the class of functions (66).

This is our "multiplicative Ward identity" for the case that $f$ has zeros and poles. What remains is to put it in a more tractable form, which we will do by projecting the "infinite ends" of Fig. $5 \mathrm{~b}$ back to finite points, whereupon the exotic restriction (66) on the allowed class of functions will show up as operator insertions at the points $P_{i}$ and $Q_{j}$ where $f$ has poles or zeros.

We first need a more concrete understanding of the exotic condition (66). To this aim, focus on a particular point $P$ at which $f$ has a single pole. Let $z$ be a local holomorphic function with a simple zero at $P$. (Thus, $f \sim z^{-1}$ near $P$.) Instead of removing from $\Sigma$ the single point $P$, let us remove a tiny disc, say the disc $D_{\varepsilon}$ defined by $|z|<\varepsilon$. At this point we need a boundary condition on the values of $\psi$ on $S=\partial D$. The natural boundary condition which corresponds to (65) is that $\left.\psi\right|_{S}$ should lie in $H_{+}$, where $H_{+}$is the "standard" point in the Grassmannian with basis $\left\{1, z, z^{2}, \ldots\right\} .{ }^{11}$ Thus, $H_{+}$consists of functions with no poles if continued inside the disc. Likewise, $\left.\tilde{\psi}\right|_{S}$ should lie in $H_{+}$.

Evidently, the transformation to $\psi^{\prime}=f \psi$ and $\tilde{\psi}^{\prime}=f^{-1} \tilde{\psi}$ means that $\left.\psi^{\prime}\right|_{S}$ lies in the space $z^{-1} H_{+}$, with basis $\left\{z^{-1}, 1, z, z^{2}, \ldots\right\}$ and $\left.\tilde{\psi}^{\prime}\right|_{S}$ lies in the space $z H_{+}$with basis $\left\{z, z^{2}, \ldots\right\}$.

Now, let us interpret this "physically." The functions $\left\{1, z, z^{2}, \ldots\right\}$ which are a basis for $H_{+}$are the wave functions of filled states of $\psi$ particles in the negative energy Dirac sea. In $z^{-1} H_{+}$, spanned by $\left\{z^{-1}, 1, z, \ldots\right\}$, there is one more filled state, with wave function $z^{-1}$. This is clearly the wave function for the positive energy state of lowest energy. In conformal field theory, there is of course a vertex operator for every state. The vertex operator for a state in which the first positive energy excitation has been added to the vacuum is simply $\psi$.

Thus, up to normalization, the exotic condition (66) at a point $P$ where $f$ has a pole is just an instruction to insert a factor $\psi(P)$. We must worry about the normalization, however, since the conformal spin of $\psi$ is not 0 but $1 / 2$. From the data at our disposal - a function $f$ with a simple pole at $P$ - the only other factor of

${ }^{11}$ The role of $H_{+}$and $H_{-}$is reversed compared to Sect. (1), because now we are working on the region $|z|>\varepsilon$, while in Sect. (1) we were working on the Riemann surface $|z|<1$ 
spin $1 / 2$ that we can form is $\sqrt{\frac{d\left(f^{-1}\right)}{d z}}$. So it must be that the factor to be inserted at a pole of $f$ is $\psi(P)) \cdot\left(\left.\frac{d f^{-1}}{d z}\right|_{P}\right)^{-1 / 2}$. Apart from the sign, which we will not try to clarify (and which is clearly linked to minus signs associated with fermi statistics), this is invariant under reparametrization of $z$, since $\psi$ transforms like $\left(\frac{d}{d z}\right)^{1 / 2}$.

It is now easy to see what happens at a point $Q$ where $\psi$ has a simple zero. Pick a uniformizer $z$ at $Q$, and remove the disc $D_{\varepsilon}$ given by $z<\varepsilon$. Let $\psi^{\prime}=f \psi$. On $S=\partial D$, $\psi^{\prime}$ lies in the space $z H_{+}$spanned by $\left\{z, z^{2}, \ldots\right\}$. The missing function 1 means that a negative energy particle has been removed from the Dirac sea or in other words an antiparticle has been added. The appropriate vertex operator is $\tilde{\psi}$ or more exactly - allowing for normalization - it is $\tilde{\psi}(Q)\left(\left.\frac{d f}{d z}\right|_{Q}\right)^{-1 / 2}$.

Thus we arrive at the definitive statement of our multiplicative Ward identities. The transformation $\psi \rightarrow f \psi, \tilde{\psi} \rightarrow f^{-1} \tilde{\psi}$, in addition to shifting boundary conditions from $W$ to $f W$, also brings about the operator insertions just described. So

$$
\int_{W} \mathscr{D} \psi \mathscr{D} \tilde{\psi} \exp -I=\int_{f W} \mathscr{D} \psi \mathscr{D} \tilde{\psi} e^{-I} \cdot \prod_{P_{\imath}} \frac{\psi\left(P_{i}\right)}{\sqrt{\left(\frac{d f^{-1}}{d z}\right)_{P_{i}}}} \prod_{Q_{\jmath}} \frac{\tilde{\psi}\left(Q_{j}\right)}{\sqrt{\left(\frac{d f}{d z}\right)_{Q_{J}}}} .
$$

Here $P_{i}$ and $Q_{j}$ run over the poles and zeros of $f$, which we have assumed to be simple. It is easy, though, to generalize the formula to allow for $k$-fold poles or zeros. A $k$-fold pole would give

$$
\prod_{j=0}^{k-1} \partial^{(j)} \psi(P) \cdot\left(\frac{d f^{-1 / k}}{d z}\right)^{-k^{2} / 2}
$$

while a $k$-fold zero gives

$$
\prod_{j=0}^{k-1} \partial^{(j)} \tilde{\psi}(Q)\left(\frac{d f^{1 / k}}{d z}\right)^{-k^{2} / 2}
$$

Since (67) may seem unfamiliar, and the derivation could be faulted for lack of rigor, we will now pause to check (67) explicitly for the simplest case in which $\Sigma$ is the Riemann sphere - without boundary. For $f$ we take a general rational function

$$
f(z)=\prod_{j=1}^{N} \frac{z-Q_{j}}{z-P_{j}}
$$

The identity (67) reads

$$
1=\prod_{j=1}^{N}\left(\left(\frac{\partial f^{-1}}{\partial z}\right)_{P_{j}}\left(\frac{\partial f}{\partial z}\right)_{Q_{j}}\right)^{-1 / 2} \cdot\left\langle\prod_{j=1}^{N} \psi\left(P_{j}\right) \prod_{k=1}^{N} \tilde{\psi}\left(Q_{j}\right)\right\rangle,
$$

or in other words

$$
\frac{\prod_{i \neq j}\left(P_{i}-P_{j}\right) \prod_{i^{\prime} \neq j^{\prime}}\left(Q_{i^{\prime}}-Q_{j^{\prime}}\right)}{\prod_{k, l}\left(P_{k}-Q_{l}\right)}=\left\langle\prod_{j=1}^{N} \psi\left(P_{j}\right) \prod_{k=1}^{N} \tilde{\psi}\left(Q_{k}\right)\right\rangle .
$$


The right-hand side of (72) can be evaluated in terms of the propagator $\langle\psi(P) \tilde{\psi}(Q)\rangle=\frac{1}{P-Q}$. It is

$$
\sum_{\pi}(-1)^{\pi} \prod_{j=1}^{N} \frac{1}{P_{j}-Q_{\pi(j)}},
$$

where $\pi$ is a permutation of $N$ objects, the sum runs over all permutations, and $(-1)^{\pi}$ is \pm 1 for even or odd permutations. The left-and right-hand sides of $(72)$ can be seen to have the following properties. Both have simple zeros where $P_{i}=P_{j}$ or $Q_{i}=Q_{j}$, and both have as their only singularities a simple pole at $P_{i}=Q_{j}$ with residue a function of the same kind with one less pole and one less zero. These properties characterize the left and right hand sides of (72) completely, verifying the claimed equality.

The absolute value squared of (72) is often cited as a basic formula in bosonization of fermions, which lately has been treated in a geometric setting [13]. Thus, if $\phi$ is a free bose field with propagator $\langle\phi(P) \phi(Q)\rangle=-\ln |P-Q|^{2}$ one computes

$$
\left\langle\prod_{i=1}^{N} \exp i \phi\left(P_{i}\right) \prod_{j=1}^{N} \exp -i \phi\left(Q_{j}\right)\right\rangle=\frac{\prod_{i \neq j}\left|P_{i}-P_{j}\right|^{2} \prod_{i^{\prime} \neq j^{\prime}}\left|Q_{i^{\prime}}-Q_{j^{\prime}}\right|^{2}}{\prod_{k, l}\left|P_{k}-Q_{l}\right|^{2}} .
$$

On the other hand, if we introduce left moving as well as right moving fermions, then the absolute value squared of (72) is

$$
\left\langle\prod_{j=1}^{N} \bar{\psi}\left(1+\gamma_{5}\right) \psi\left(P_{j}\right) \prod_{k=1}^{N} \bar{\psi}\left(1-\gamma_{5}\right) \psi\left(Q_{k}\right)\right\rangle \text {. }
$$

We have extracted the equality of (74) and (75) as a special case of a much more general multiplicative Ward identity for which we have given a conceptual explanation. It is in this sense that our considerations shed light on the phenomenon of bosonization of fermions.

In case $f$ has one zero and one pole, (67) reduces to

$$
\frac{1}{\sqrt{\left(\frac{d f^{-1}}{d z}\right)_{P}\left(\frac{d f}{d z}\right)_{Q}}}\langle\psi(P) \tilde{\psi}(Q)\rangle_{f W}=\frac{P f \bar{D}_{W}}{\operatorname{Pf} \bar{D}_{f W}} .
$$

Upon substituting $W \rightarrow f^{-1} W$, this becomes

$$
\frac{1}{\sqrt{\left(\frac{d f^{-1}}{d z}\right)_{P}\left(\frac{d f}{d z}\right)_{Q}}}\langle\psi(P) \tilde{\psi}(Q)\rangle_{W}=\frac{\operatorname{Pf} \bar{D}_{f^{-1} W}}{\operatorname{Pf} \bar{D}_{W}} .
$$

Here $G(P, Q)=\langle\psi(P) \tilde{\psi}(Q)\rangle$ is the Dirac propagator with boundary conditions $W$. It is uniquely characterized by the following: ${ }^{12}$

\footnotetext{
${ }^{12}$ In what follows, we work on the Cartesian product $\Sigma \times \Sigma ; L_{1}$ and $L_{2}$ denote the canonical line bundles of the "first" and "second" copies of $\Sigma$
} 
(1) $G(P, Q)$ is a meromorphic section of $L^{1 / 2} \otimes L^{1 / 2}$ (or more precisely of $L_{1}^{1 / 2} \otimes L_{2}^{1 / 2}$ ) over $\sum \times \sum$ whose only singularity is a simple pole at $P=Q$ with residue one. (This notion of "residue" makes some since the restriction of $L^{1 / 2} \otimes L^{1 / 2}$ to the diagonal in $\Sigma \times \Sigma$ is $L$.)

(2) Regarded as a function of $P$ for fixed $Q$ (or $Q$ for fixed $P$ ) the restriction of $G(P, Q)$ to $S=\partial \Sigma$ lies in $W$ (or its dual $\tilde{W})$.

The first condition here is just the short distance behavior of the Dirac propagator, and the second was discussed in Sect. (1) [see Eq. (15)].

The relation (67) between the propagator and the determinant holds on any Riemann surface. If we specialize to the case in which $\Sigma$ is a Riemann sphere with one hole cut out, then (76) is the relation between the Baker function and the tau function as developed by Segal and Wilson in [1]. To compare with their results, note the following. They work in the region $|z| \geqq 1$ in the complex plane, and take $P=\zeta, Q=\infty$. A function with a single pole at $P$ and zero at $Q$ is then (in the notation of [1]) $q_{\zeta}^{-1}(z)=(1-z / \zeta)^{-1}$. The "Baker function"13 $\tilde{\psi}_{W}(\zeta)$ of their discussion corresponds to our $G(\zeta, \infty)$. Segal and Wilson describe $\tilde{\psi}_{W}(\zeta)$ as a "function" rather than a section of the spin bundle $L^{1 / 2}$. In doing so, they are in effect trivializing the spin bundle on the finite $z$ plane by choosing a section whose only singularity is a simple pole at $z=\infty$. Thus, when they require $\tilde{\psi}_{W}(\zeta) \rightarrow 1$ for $\zeta \rightarrow \infty$, this amounts to saying that $G(\zeta, \infty)$ has a pole of residue 1 for $\zeta \rightarrow \infty$. And the boundary condition at $|z|=1$ of Segal and Wilson is precisely our condition (2) above. Thus, with $f \leftrightarrow q_{\zeta}^{-1}$ and $\tau \leftrightarrow P f$, we recognize Eq. (76) as Proposition 5.14 of [1].

In Eq. (67), we see that operator insertions of $\psi(P)$ and $\tilde{\psi}(Q)$ arise at poles and zeros of a meromorphic function $f$. It is natural to expect that a more general divisor

$$
\Sigma P_{i}-\Sigma Q_{j}
$$

(which is not necessarily the principal divisor of a global meromorphic function) will similarly be related to operator insertions of $\psi\left(P_{i}\right)$ and $\tilde{\psi}\left(Q_{j}\right)$. Let $E$ be the line bundle with divisor (78). The ordinary Dirac propagator $G(P, Q)=\langle\psi(P) \tilde{\psi}(Q)\rangle$ (with $\psi, \tilde{\psi}$ sections of $L^{1 / 2}$ ) is - as we have already noted - the section of $\mathscr{L}=\left(L^{1 / 2}\right)_{1} \otimes\left(L^{1 / 2}\right)_{2}$ over $\Sigma \times \sum$ whose only singularity is a pole of residue one on the diagonal. $(\mathscr{L}$ is the line bundle whose restriction to either copy of $\Sigma$ is isomorphic to $L^{1 / 2}$.) If we wish to study fermions $\psi, \tilde{\psi}$ that are sections of $L^{1 / 2} \otimes E$ and $L^{1 / 2} \otimes E^{-1}$ respectively, the propagator $G_{E}(P, Q)$ would be a section of

$$
\mathscr{L}_{E}=\left(L^{1 / 2} \otimes E\right)_{1} \otimes\left(L^{1 / 2} \otimes E^{-1}\right)_{2}
$$

over $\Sigma \times \Sigma$; that is, $\mathscr{L}_{E}$ is the line bundle whose restriction to the first or second copy of $\Sigma$ is isomorphic to $\left(L^{1 / 2} \otimes E\right)$ or $\left(L^{1 / 2} \otimes E^{-1}\right)$, respectively. $G_{E}(P, Q)$ is still required to have for its only singularities a simple pole of residue one on the diagonal.

Instead of regarding $G_{E}(P, Q)$ as a section of the twisted line bundle $\mathscr{L}_{E}$ with singularity only on the diagonal, we may regard it as a section of $\mathscr{L}$ with poles at $P=Q$ or $P=Q_{j}$ and zeros at $P=P_{i}$ or $Q=Q_{j}$. (This is so because (78) is the divisor of $E$; a section of $E$ is just a function which to be regular is required to have zeros at the $P_{i}$ and permitted to have poles at the $Q_{j}$.) But we may immediately write down a

\footnotetext{
${ }^{13}$ We suppress $g$, writing $g W$ as $W$
} 
section of $\mathscr{L}$ with those properties:

$$
G_{E}(P, Q)=\left\langle\psi(P) \tilde{\psi}(Q) \prod_{i} \psi\left(P_{i}\right) \prod_{j} \tilde{\psi}\left(Q_{j}\right)\right\rangle .
$$

This simple argument expresses $G_{E}$ in terms of $G$, since the right-hand side is a free field correlation function which can of course be expressed in terms of the free propagator $G$. (The formula is simply

$$
\sum_{\pi}(-1)^{\pi} \prod_{j=0}^{\pi} G\left(P_{j}, Q_{\pi(j)}\right)
$$

where $G(P, Q)$ is the ordinary propagator, not twisted by $E$, and we have taken $P=P_{0}, Q=Q_{0}$ to simplify the notation.) The argument leading to (80) shows that the divisor of a line bundle corresponds to insertions of fermions and antifermions. This fact emerged in [13]. Its analogue for vector bundles will emerge in Sects. four and five.

Apart from the motivations already indicated above, a further reason for formulating multiplicative Ward identities is that this is a crucial step if one wishes to work in an algebraic setting, over ground fields other than the complex numbers. Multiplicative Ward identities will be one ingredient of the purely algebraic description of the theory of free fermions that we will give in Sect. (4). Before plunging into this, however, we first pause to describe the physical reasoning behind the construction.

\section{Operator Valued Differential Forms}

Our goal in this section is to express the Ward identities of conformal field theory [14] in terms of operator valued differential forms. These have already made an appearance in Sect.(1). We will only consider holomorphic conformal field theories, that is, theories in which all degrees of freedom are holomorphic. The first subsection of this section essentially explains the physical background to Sect. (4); then we continue and analyze more detailed properties of operator valued differential forms.

Consider, in a conformal field theory on some Riemann surface $\Sigma$, a conformal field $\psi$ of spin $S$. It is an "operator valued section of $L^{S}$," with $L$ being the canonical line bundle of $\Sigma$, and $L^{S}$ its $S^{t h}$ power. Let $f$ be an ordinary $c$-number meromorphic section of $L^{1-S}$. Then $f \psi$ is an operator valued section of $L$, or in other words an operator valued differential form. Notice that we permit $f$ to be meromorphic, not necessarily holomorphic; an important part of the story has to do with the poles of $f$. Let $P_{i}$ be the positions of the poles of $f$.

Let us formulate the precise properties of $f \psi$ which entitle it to this name, operator valued differential form. Let us insert $f \psi(z)$ in an arbitrary correlation function with insertions of other operators $O_{j}\left(P_{j}^{\prime}\right)$. Thus, we define.

$$
F(z)=\int \mathscr{D} X e^{-I} \cdot \prod O_{j}\left(P_{j}^{\prime}\right) \cdot f \psi(z) .
$$

Here $\mathscr{D} X$ is a shorthand expression for integration over the unspecified field variables of the conformal field theory in question. Now, the correlation function $F(z)$ is an ordinary $c$-number differential form, which in a holomorphic conformal 
field theory will be holomorphic except for certain poles. It will have poles at the $P_{i}$, where $f$ has poles, and it may have poles at the $P_{j}^{\prime}$, because of possible short distance singularities in the operator products $\psi(z) \cdot O_{j}\left(P_{j}^{\prime}\right)$ for $z \rightarrow P_{j}^{\prime}$. What we mean in saying that $f \psi(z)$ is an "operator valued differential form" is precisely this: its correlation functions are ordinary meromorphic differential forms, with the poles just described.

Let us delete the positions $P_{i}$ and $P_{j}^{\prime}$ of the poles from $\Sigma$ to make an incomplete Riemann surface $\Sigma^{\prime}$ on which $F(z)$ is holomorphic, i.e., obeys $\bar{\partial} F(z)=0$. Then

$$
0=\int_{\Sigma^{\prime}} \bar{\partial} F(z)=\sum_{\alpha} \oint_{C_{\alpha}} F(z),
$$

with $\alpha$ running over the poles of the differential form $F(z)$, and $C_{\alpha}$ a contour that encloses the $\alpha^{\text {th }}$ pole. Equation (83) is simply the statement that the sum of the residues of the differential form $F(z)$ is zero, i.e.,

$$
\sum_{\alpha} \operatorname{Res}_{\alpha}(F)=0
$$

the residues being defined by

$$
\operatorname{Res}_{\alpha}(F(z))=\frac{1}{2 \pi i} \oint_{C_{\alpha}} F(z) .
$$

Equation (84) is of course a fundamental statement about meromorphic differential forms on a Riemann surface and clearly has nothing to do with the interpretation of $F(z)$ as a correlation function. The real story begins when one supplies the geometrical interpretation of the residues of $F(z)$.

We have considered two types of point at which $F(z)$ has poles - points $P_{j}^{\prime}$ at which a vertex operator $O_{j}$ was inserted, and points $P_{i}$ at which the pole is entirely due to a pole in $f$. We would like to discuss these two cases uniformly. To do so, let us adopt the convention that a vertex operator is inserted at each of the $P_{i}$ and $P_{j}^{\prime}$, but this vertex operator happens to be the identity operator 1 in the case of the $P_{i}$. After all, 1 is a perfectly respectable vertex operator in conformal field theory - it represents the coupling to an $S L(2, R)$ invariant "vacuum" state. Including 1 as one of our vertex operators, we henceforth treat the $P_{i}$ and $P_{j}^{\prime}$ on an equal footing.

As in Fig. 6c, let us project to infinity one of the points $P_{\alpha}$ at which $F$ has a pole. In the infinite past, i.e., far out on the cylinder of Fig. 6c, there will then appear a quantum state $|\Lambda\rangle$. It is simply the state whose vertex operator is $O_{\alpha}$. (If $O_{\alpha}=1$, then $|\Lambda\rangle$ is the "vacuum.") $|\Lambda\rangle$ is a state in the Hilbert space $H_{P_{\alpha}}$ of possible "in" states at $P_{\alpha}$. We can give a more physical description of the right-hand side of (85). It is

$$
\operatorname{Res}_{\alpha} F(z)=\int \mathscr{D} X e^{-I} \prod_{j \neq \alpha} O_{j}\left(P_{j}\right) \cdot\left(\frac{1}{2 \pi i}\right) \oint_{C_{\alpha}}(f \psi)|\Lambda\rangle .
$$

The notation in (86) is somewhat hybrid. Writing the state $|\Lambda\rangle$ as part of the argument of the path integral is meant as a reminder that the boundary condition at $P_{\alpha}$ is that the state $|\Lambda\rangle$ is coming in from the far past. At the $P_{j}$ with $j \neq \alpha$, which are of no interest at the moment, we have projected the punctures back to finite points and restored the vertex operators $O_{j}$. The contour $C_{\alpha}$ surrounds $P_{\alpha}$.

Now we must recall the correspondence between Feynman path integrals and operators. The object $\psi$ inserted in a path integral corresponds in the Hamiltonian 
(a)

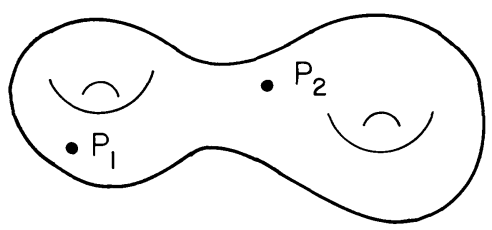

(b)
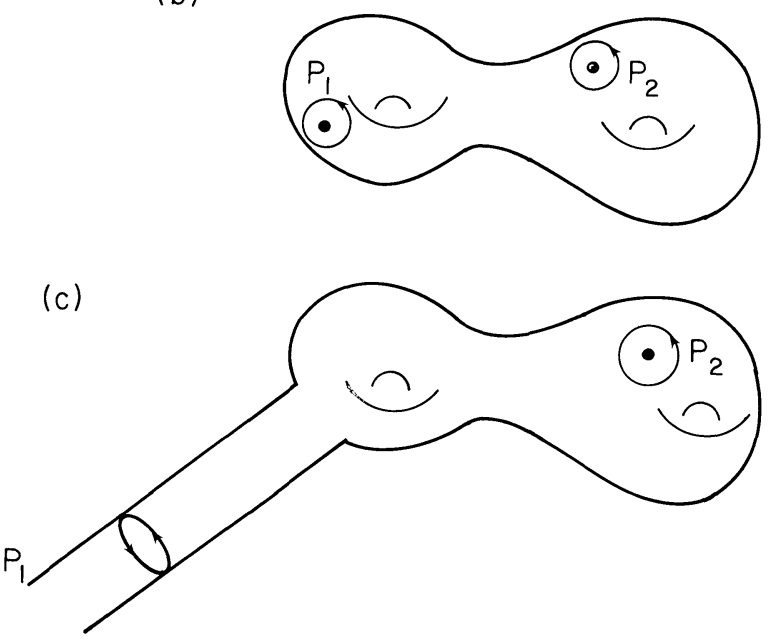

Fig. 6a-c. Poles of the correlation function $F(z)$ are indicated in a. Upon deleting the positions of these poles from $\Sigma$, we can write a contour integral formula picking up contributions from the "operator-valued residues," as in $\mathbf{b}$. Projecting the position of one of the former poles to infinity, as in c, we see that the operator valued residues are really moments of suitable operators. A contour $C$ surrounding one of the poles of $F$ is sketched in $\mathbf{b}$ and again in $\mathbf{c}$

description to an operator, which we will also call $\psi$, acting in the quantum Hilbert space $\mathscr{H}_{P_{\alpha}}$. The factor $\oint(f \psi)$ in (86) is simply an instruction to take the boundary condition at $P_{\alpha}$ to correspond not to insertion of $|\Lambda\rangle$, but rather to insertion of

$$
\frac{1}{2 \pi i} \oint(f \psi)|\Lambda\rangle \text {. }
$$

The analogy with (85) suggests that the operator which appears in (87) should be regarded as the operator valued residue of the operator valued differential form $f \psi$. Thus, we define the residue of $f \psi$ at $P$ to be the operator

$$
\operatorname{Res}_{P}(f \psi)=\frac{1}{2 \pi i} \oint_{C}(f \psi),
$$

regarded as an operator on the space of possible quantum states (or equivalently possible vertex operators) at $P ; C$ is a contour surrounding $P$ but no other possible poles.

Of course, whatever can be said about states can be restated in terms of operators. If the state $|\Lambda\rangle$ corresponds to the vertex operator $O_{P}$, then the state 
$\operatorname{Res}_{P}(f \psi)|\Lambda\rangle$ will correspond to some other vertex operator which we may as well call $\operatorname{Res}_{P}(f \psi) \cdot O(P)$. Thus, we have a residue operation acting on operators,

$$
O(P) \rightarrow \operatorname{Res}_{P}(f \psi) \cdot O(P),
$$

which has neither more nor less content than (88). In this section, we wish to formalize the basic properties of the residue operation. We can now state the first principle:

(i) For $O(P)=1, \operatorname{Res}_{P}(f \psi) \cdot 1=0$ unless $f$ has a pole at $P$.

This is just a restatement of the fact that the poles in (82) (which are the only points at which the residues may be nonzero) only occur at the $P_{i}$ (where $f$ has poles) or the $P_{j}^{\prime}$ (where $O \neq 1$ ).

Consider now a product of observeables

$$
O_{1}\left(P_{1}\right) O_{2}\left(P_{2}\right) \ldots O_{N}\left(P_{N}\right) \text {. }
$$

We have written this as a finite product, but one could equally well think of it as an infinite product

$$
\prod_{P \in \Sigma} O_{P}(P)
$$

which runs over all points in $\Sigma$ with the agreement that $O_{P}(P)=1$ for all but finitely many $P$. We will now in a trivial way extend the notion of $\operatorname{Res}_{P}(f \psi)$ so that it acts not just on operators at $P$ but on arbitrary products (91). We simply declare that $\operatorname{Res}_{P}(f \psi)$ will be considered to act as before on the $P$ component of (91) while leaving the other components invariant,

$$
\operatorname{Res}_{P}(f \psi) \cdot \prod_{Q} O_{Q}(Q)=\left(\operatorname{Res}_{P}(f \psi) \cdot O_{P}(P)\right) \cdot \prod_{Q \neq P} O_{Q}(Q) .
$$

Having defined $\operatorname{Res}_{P}(f \psi)$ for arbitrary $P$ as an operator acting on arbitrary products of observables, we now wish to study the "sum of the residues." Thus, let

$$
\mathscr{T}=\left(\sum_{P} \operatorname{Res}_{P}(f \psi)\right) \cdot \prod_{Q} O_{Q}(Q) .
$$

It is important to note that (93) is a well-defined finite sum, since (92) vanishes unless $f$ has a pole at $P$ or $O_{P} \neq 1$.

We would now like to find the appropriate statement about operator valued differential forms which generalizes the statement that the sum of the residues of an ordinary $c$-number differential form is zero. Let us think of the Feynman path integral as defining a linear functional on the space of observables. Thus, for an arbitrary observable $\prod_{P} O_{P}(P)$, we will abbreviate

$$
\int \mathscr{D} X e^{-I} \prod_{P} O_{P}(P)
$$

as

$$
\int \prod_{P} O_{P}(P)
$$

with $\int$ being a linear functional from observables to complex numbers. Then (84) is equivalent to the statement that

$$
0=\int\left(\sum_{P} \operatorname{Res}_{P}(f \psi)\right) \prod_{Q} O_{Q}(Q)
$$


We have in fact defined the notion of the operator valued residue precisely so as to be able to express (84) in the form (96).

We thus have our second axiom for operator valued differential forms:

(ii) The expectation value of the sum of the residues of an operator valued differential form is zero, in the sense that (96) vanishes for all observables $\prod_{Q} O_{Q}(Q)$.

Equation (96) is a restatement of the usual (additive) Ward identities of conformal field theory, in terms of operator valued differential forms. The connection with the formulation of [14] is roughly that they discuss the operator product expansion

$$
(\psi(z) \cdot O(w))_{z \rightarrow w} \sim \sum_{n \geqq-N} O_{n} \cdot(z-w)^{n}
$$

and must, in principle, keep track of all terms on the right-hand side. [The correlation functions are in a sense determined just by the terms of $n<0$, but the associativity conduction of the operator product involves all terms in (97).] We on the other hand consider products

$$
f(z) \psi(z) \cdot O(w)
$$

with arbitrary $f$, and we extract only the residue, that is, the coefficient of $(z-w)^{-1}$. Clearly, by picking $f(z) \sim(z-w)^{-n-1}$, the operator $O_{n}$ of (97) will appear as the residue $\operatorname{Res}_{w}(f \psi) \cdot O(w)$, so by considering residues of operator valued differential forms, we in fact repackage all of the information of the usual Ward identities. This means that like the Ward identities of [14], our Ward identity (ii) is powerful enough to determine the correlation functions (that is, the linear functional $f$ ). Since (ii) has no content beyond the standard Ward identities, the only issue is whether one will obtain new insight by thinking in terms of operator valued differential forms and their residues.

(ii) is recognizably a generalization of a conventional statement about residues of $c$-number differential forms. Another fundamental property of $c$-number differential forms is that the residues of an exact form

$$
\omega=d \lambda
$$

are all zero. We would like to exhibit the statement analogous to (99) in the context of the operator valued differential forms of conformal field theory. To do so, the main obstacle is that we must explain the analogue of the exterior derivative " $d$ " which appears in the formula $\omega=d \lambda$. Here $\lambda$ is of course an ordinary $c$-number function, and $d$ is the natural flat connection on scalar functions. To generalize $d$ to operator valued scalars, it is necessary to show that operator valued scalars are in a suitable sense sections of a flat vector bundle. We thus must plunge into a long digression about the bundle of observables in a conformal field theory on a Riemann surface.

\section{The Bundle of Observables}

We will have to systematically discuss arbitrary local observables, which are not necessarily conformal fields. Let $P$ be a point in $\Sigma$, and $V_{P}$ the space of all local operators that can be inserted at $P$. Except for conformal fields, most operators in 
$V_{P}$ do not have a definite dimension. For instance, the energy momentum tensor $T(P)$ is a fundamental example of a field that is not a conformal field. Under global scale transformations, $T(P)$ transforms as an operator of dimension two, but under a general change in local parameter at $P, T(P)$ shifts by a multiple of the identity operator (which has dimension zero), so we describe $T(P)$ as an operator of dimension at most two.

To put this a little bit differently, what would be described in one coordinate system as a multiple of the energy momentum tensor, say $\alpha T(P)$ (with $\alpha$ a complex number), would be described in another coordinate system as a linear combination of the energy momentum tensor and a $c$-number, say

$$
\beta^{\prime}+\alpha^{\prime} T(P) \text {. }
$$

This means that in an invariant way, without choice of a coordinate system, one cannot think about "inserting the energy momentum tensor at a point $P$ on the Riemann surface $\Sigma$." One must always think in terms of inserting a linear combination of the pair of operators 1 (the identity operator) and $T(P)$. Clearly, such a linear combination does not have a definite dimension, but it has maximum dimension two. To illustrate more fully some of the ideas of the present section and to make contact with [19], the system $(1, T)$ will be discussed more fully in an appendix.

For convenience, we will assume in what follows that only the identity operator has dimension zero and that all other dimensions are positive integers. Let $V_{P, n}$ be the subspace of $V_{P}$ consisting of operators of dimensian at most $n$. Clearly, $V_{P, n}$ $C V_{P, n+1}$, and

$$
V_{P}=\bigcup_{n} V_{P, n}
$$

Equation (101) is a filtration of $V_{P}$ by subspaces of finite dimension and is canonical - it does not depend on a choice of local parameter at $P .{ }^{14}$ If one chooses a local parameter $z$ at $P$ and defines the dimension of a field in terms of the transformation under the vector field $z(d / d z)$ ("a global scaling near $P$ "), then it is possible to define a definite dimension to the fields (for instance, $T(P)$ has dimension two). Thus, upon choosing a local parameter, we get a direct sum decomposition

$$
V_{P}=\oplus_{n} W_{P, n},
$$

where $W_{P, n}$ consists of fields that transform with weight $n$ under $z(d / d z) .{ }^{15}$

In interesting holomorphic conformal field theories, the $V_{P, n}$ are finite dimensional, but $V_{P}$ is always infinite dimensional (since including descendants there are necessarily infinitely many fields).

As $P$ varies in $\Sigma$, the $V_{P}$ are fibers of a holomorphic vector bundle $\mathscr{V} *$ over $\Sigma .{ }^{16}$ The $V_{P, n}$ are likewise fibers of subbundles $\mathscr{V}_{n}^{*}$. Clearly,

$$
\mathscr{V}^{*}=\bigcup_{n=0}^{\infty} \mathscr{V}_{n}^{*} .
$$

\footnotetext{
${ }^{14}$ A local parameter $z$ at a point $P$ on a Riemann surface is simply a function $z$ which is holomorphic in a neighborhood of $P$ and has a simple zero at $P$

${ }^{15}$ One can interpret $W_{P, n}$ as the quotient $V_{P, n} / V_{P, n-1}$, but there is no canonical splitting $V_{P, n}=V_{P, n-1} \oplus W_{P, n}$. The obstruction is that fields such as the energy momentum tensor which are not conformal fields mix under change of local parameter with fields of lower dimension $16 \mathscr{V}^{*}$ is the dual of $\mathscr{V}$, which will appear later
} 
Again, (103) is a filtration, and there is no canonical direct sum decomposition that induces it.

What is the structure group of $\mathscr{V}^{*}$ ? Let us answer this on physical grounds. Let $\mathscr{H}$ be the Hilbert space of the conformal field theory under discussion. Given any point $P$ on a Riemann surface, the space $V_{P}$ of local operators that can be inserted at $P$ is isomorphic to $\mathscr{H}$, but there is no canonical way to make this isomorphism. To identify $V_{P}$ with $\mathscr{H}$, one needs a local parameter $z$ at $P$. Given such a local parameter, one has a natural way to project $P$ to infinity, say by choosing a metric

$$
d s^{2}=\frac{d z d \bar{z}}{z \bar{z}} .
$$

In the metric (104), the Riemann surface $\Sigma$ is a flat cylinder (at least in a neighborhood of $P$ where the local parameter $z$ is well behaved), and $P$ has been projected to the "far past," as in Fig. 6c. This gives us an identification with the canonical formalism, or in other words a specific identification of $V_{P}$ with $\mathscr{H}$. form

If $z$ is a local parameter at $P$, then another local parameter $z^{\prime}$ would be of the

$$
z^{\prime}=a_{1} z+a_{2} z^{2}+a_{3} z^{3}+\ldots
$$

with $a_{1} \neq 0$. Such changes of coordinate are generated by the vector fields $z^{n+1}(d / d z), n=0,1,2, \ldots$. These correspond to the usual Virasoro generators $L_{n}$, with $n \geqq 0$. If we denote the Lie algebra of the $L_{n}, n \geqq 0$ as $\mathscr{R}$, and the group generated by $\mathscr{R}$ as $R$, then it is natural to call $R$ the group of changes of local parameter. The fact that an identification of the fiber of $\mathscr{V}^{*}$ with a standard space $\mathscr{H}$ arises on choice of a local parameter means that $R$ is the structure group of $\mathscr{V}^{*}$.

But in fact, $\mathscr{V}^{*}$ is much more rigid than a generic vector bundle with structure group $R$. One side of this has to do with group theory. Let $\mathscr{R}^{+}$be the Lie algebra of the $L_{n}, n \geqq-1$, and let $\widehat{\mathscr{R}}$ be the Virasoro algebra generated by all of the $L_{n}, n \in Z$. The fiber $V_{P}$ of a vector bundle with structure group $Y$ must be a representation of the Lie algebra $\mathscr{R}$, but there is no general reason that that representation must extend to a representation of $\mathscr{R}^{+}$or $\widehat{\mathscr{R}}$. But in conformal field theory, $V_{P}$ is actually a representation of $\widehat{\mathscr{R}} . \mathscr{R}^{+}$is about to play a crucial role, but $\widehat{\mathscr{R}}$ will not (except in the appendix). This is one reflection of the fact that something crucial is missing in the present discussion (as from other existing discussions of conformal field theory).

The other side of the rigidity of $\mathscr{V}^{*}$ has to do with the following. If indeed $z$ is a local parameter at $P$, then for all $Q$ in some neighborhood of $P, z-z(Q)$ is a local parameter at $Q$, and the choice of metric

$$
d s^{2}=\frac{d z d \bar{z}}{(z-z(Q))(\bar{z}-\bar{z}(Q))}
$$

leads just as in our discussion of $V_{P}$ to an identification of $V_{Q}$ with $H$. Thus, choosing a local parameter at $P$ gives a trivialization of $\mathscr{V}^{*}$ not just at $P$ but in a whole neighborhood of $P$.

Pick a local parameter $z$ at $P$ and pick a basis of operators $\left\{\psi^{k}\right\}$ in the space $V_{P}$ of operators at $P$. For instance, in a theory of free fermions, these may be

$$
1, \psi(P), d \psi(P), \psi d \psi(P), d^{2} \psi(P),
$$


etc., with " $d$ " being $d / d z$. As described above, the choice of parameter $z$ gives a trivialization of $\mathscr{V}^{*}$ in a neighborhood $\Sigma_{0}$ of $P$, so we can erase the argument " $(P)$ " in (107); without any further arbitrary choices, we have in a natural way the basis of operators $1, \psi, d \psi, \psi d \psi, d^{2} \psi, \ldots$ at any point in $\Sigma_{0}$.

In this section we have been discussing "operator valued differential forms." We now wish to broaden our horizons a bit and discuss "operator valued meromorphic functions." An operator valued meromorphic function $A(z)$ is an object with the property that its correlation functions are ordinary meromorphic functions. After trivializing $\mathscr{V}^{*}$ in a neighborhood $\Sigma_{0}$, a general operator-valued meromorphic function $\alpha$ can be expanded

$$
\alpha=\sum a_{k} \cdot \psi^{k}
$$

with the $a_{k}$ being ordinary meromorphic functions in $\Sigma_{0}$.

We now want to define the "exterior derivative" $d \alpha$ of the operator valued meromorphic function $\alpha$. Proceeding blindly, we write

$$
d \alpha=\sum d a_{k} \cdot \psi^{k}+\sum a_{k} \cdot d \psi^{k} .
$$

By $d a_{k}$ we mean (in the $z$ coordinate system) simply $d a_{k} / d z$. But what is $d \psi^{k}$ ? It is crucial now that the $\psi^{k}$ are a basis for all local operators, and this includes all derivatives of local operators, as in (107). Therefore, the $d \psi^{k}$ are simply linear combinations of the $\psi^{m}$ with constant coefficients, say

$$
d \psi^{k}=\sum_{m} w_{k, m} \psi^{m}
$$

for some constants $w_{k, m}$. Thus, (109) can be rewritten

$$
d \alpha=\sum_{m}\left(d a_{m}+\sum_{k} a_{k} w_{k, m}\right) \psi^{m},
$$

and in coordinates this is our formula for the exterior derivative of an operator valued meromorphic function.

Let $\mathscr{A}$ be the space of operator valued meromorphic functions, and $\mathscr{K}$ the space of operator valued meromorphic differential forms. We want to interpret (111) as a natural definition of an exterior derivative

$$
d: \mathscr{A} \rightarrow \mathscr{K}
$$

with the property that if $f$ is an ordinary meromorphic function, and $\alpha$ an operator valued meromorphic function, then

$$
d(f \alpha)=d f \cdot \alpha+f \cdot d \alpha,
$$

with $d f$ the ordinary exterior derivative of functions. What is missing at the moment is that the definition of (111) seemingly depended on a choice of a local parameter $z$ at a point $P \in \Sigma$. We would like to show that there is an intrinsically defined exterior derivative which in coordinates takes the form (111).

First of all, the question can be reinterpreted in the following way. While we have spoken roughly of the $a_{k}$ in (108) as "functions," it is clear that this depended on a choice of trivialization of $\mathscr{V}^{*}$, and hence the correct global description is that the family $\left\{a_{k}\right\}$ defines a global meromorphic section of the dual bundle $\mathscr{V}$. We 
have arranged to call this bundle $\mathscr{V}$, reserving the secondary name $\mathscr{V}^{*}$ for the "bundle of operators", because in a sense it is the $a_{k}$ that one really wishes to study. In formulas like (108), the $\psi^{k}$ play a bookkeeping role. If we were not guided by physical intuition, we might very well have defined $\mathscr{V}$ and neglected to mention the $\psi^{k}$ altogether.

Let $\Gamma(\mathscr{V})$ be the space of meromorphic sections of $\mathscr{V}$ and $\Gamma(\mathscr{V} \otimes L)$ the space of meromorphic sections of $\mathscr{V} \otimes L$. These correspond to the spaces of operator valued meromorphic functions and operator valued meromorphic differential forms, respectively. Equation (111) is equivalent to a "holomorphic connection"

$$
D: \Gamma(\mathscr{V}) \rightarrow \Gamma(\mathscr{V} \otimes L)
$$

obeying

$$
D(f \varrho)=d f \cdot \varrho+f \cdot D \varrho
$$

for $\varrho \in \Gamma(\mathscr{V})$ and $f$ an ordinary meromorphic function. In coordinates, $D$ is defined by (111) with the symbols $\psi^{k}$ erased. An element of $\mathscr{A}$ is locally a collection of functions $\left\{a_{k}\right\}$, and $D$ of this collection is

$$
D\left(\left\{a_{k}\right\}\right)=\left\{d a_{k}+\sum_{r} a_{r} w_{r, k}\right\} .
$$

A holomorphic connection on a holomorphic vector bundle on a Riemann surface is automatically flat, since the dimension of the Riemann surface is one in the holomorphic sense, and there is no room for curvature. Thus we are asserting among other things that the bundle $\mathscr{V}$ is naturally flat.

It is possible to convince oneself that (111) and (116) are intrinsically defined by thinking about how things transform under change of local parameter. However, a much more incisive account has been given by D. Kazhdan, and I will follow his treatment. First of all, the crucial step in making sense of (111) was (110), which is the statement that the space of all operators is closed under the operation of differentiation. The derivative of an operator is the application of the Virasoro vector field $d / d z$, which corresponds to $L_{-1}$. Thus, we recall that the structure group of $\mathscr{V}$ is $R$, generated by the Lie algebra $\mathscr{R}$ of $L_{n}, n \geqq 0$. The fiber $W_{P}$ of $\mathscr{V}$ necessarily admits a $\mathscr{R}$ action, but this in fact extends to an action of $\mathscr{R}^{+}$, generated by $L_{n}, n \geqq-1$, and this is the basis for (110) and thus for our other statements. In constructing the holomorphic connection $D$ on the bundle $\mathscr{V}$, we must expect to use the $\mathscr{R}^{+}$action on the fibers.

The other key property of $\mathscr{V}$ is that it is trivialized upon picking a local parameter $z$ at a point $P \in \sum$. Such a choice gives a canonical identification of the fiber $W_{P}$ with the Hilbert space $\mathscr{H}$ of the conformal field theory. ${ }^{17}$ Thus, let $\hat{\Sigma}$ be the space of pairs $(P, z)$ consisting of a point $P \in \Sigma$ and a local parameter $z$ at $P . \hat{\Sigma}$ is fibered over $\Sigma$, and the pullback $\hat{\mathscr{V}}$ of $\mathscr{V}$ from $\Sigma$ to $\hat{\Sigma}$ is canonically isomorphic to the product $\hat{\Sigma} \times \mathscr{M}$.

Vector fields $f(z)(d / d z)=z^{n}(d / d z)$ with $n \geqq 0$ act on $\hat{\Sigma}$ by moving the point $P$ and shifting the local parameter. Explicitly, the action is $\delta P=-f(0), \delta z=f(z)$; this

${ }^{17}$ Since we have taken a dual from operators to coefficients of operators, it is $\mathscr{H}^{*}$ that really appears here, but this is naturally isomorphic to $\mathscr{H}$ as $\mathscr{H}$ is a Hilbert space 
preserves the requirement $z(P)=0$. This gives an action of the $\mathscr{R}^{+}$Lie algebra on $\hat{\Sigma}$. We will refer to the vector fields $z^{n}(d / d z)$ with the action of $\hat{\Sigma}$ just described as $L_{n-1} \cdot{ }^{18}$ There is no way to exponentiate the $\mathscr{R}^{+}$Lie algebra action to get a group action on $\hat{\Sigma}$. However, if we restrict to the subalgebra $\mathscr{R}$ of $\mathscr{R}^{+}$consisting of vector fields that leave $P$ fixed (while transforming the local parameter at $P$ ), then the Lie algebra action exponentiates to an action of the group $R$. A section of $\mathscr{V}$ over $\Sigma$ is the same as an $R$-invariant section of the trivial bundle $\hat{\mathscr{V}}$ over $\hat{\Sigma}$, where the $R$ action on sections of $\hat{\mathscr{V}}$ is the diagonal action on the two factors in the product $\widehat{\mathscr{V}}=\hat{\Sigma} \times \mathscr{H}$. Thus, an operator valued function $\alpha$ on $\Sigma$ is equivalent to a section $\hat{\alpha}$ of the trivial bundle $\hat{\Sigma} \times \mathscr{H}$ over $\hat{\Sigma}$ which obeys

$$
L_{n} \hat{\alpha}=0, \quad n \geqq 0 .
$$

The canonical line bundle $L$ of $\Sigma$ likewise can be lifted to $\hat{\Sigma}$, where it becomes trivial, since given a pair $(P, z)$ there is a canonical basis $d z$ for the space of differential forms at $P$. Under a change of local parameter $z \rightarrow z^{\prime}=a_{1} z+a_{2} z^{2}+\ldots$, a differential form at $P$ is multiplied by $a_{1}^{-1}$. This means that a differential form $\omega$ on $\Sigma$ lifts to a function $\hat{\omega}$ on $\hat{\Sigma}$ which obeys

$$
L_{0} \hat{\omega}=\hat{\omega}, \quad L_{n} \hat{\omega}=0, \text { for } n>0 .
$$

Since $\mathscr{V}$ and $L$ lift to trivial bundles on $\hat{\Sigma}$, the same is true for their tensor product $\mathscr{V} \otimes L$. Combining (117) and (118), we see that an operator valued differential form $A$ on $\Sigma$ is the same as a section $\hat{A}$ of the trivial bundle $\hat{\Sigma} \times \mathscr{H}$ which obeys

$$
L_{0} \widehat{A}=\widehat{A}, \quad L_{n} \hat{A}=0, \text { for } n>0 .
$$

Now, to establish the geometrical nature of (111) and (116), we must show how to construct from a meromorphic section of $\mathscr{V}$ a meromorphic section of $\mathscr{V} \otimes L$. Lifting the discussion up to $\hat{\Sigma}$, the problem is to find, from an object $\hat{\alpha}$ obeying (117), an object $\hat{A}$ obeying (119). The formula is painlessly simple:

$$
\widehat{A}=L_{-1} \hat{\alpha} \text {. }
$$

From (117), (120), and the Virasoro algebra, (119) follows.

Thus, while $R$ is the structure algebra of $\mathscr{V}$, we have seen the importance of the fact that the $\mathscr{R}$ action on the fibers extends to $\mathscr{R}^{+}$. A gap in the present paper is the failure to integrate the $\widehat{\mathscr{R}}$ action into the constructions.

\section{Some More Axioms}

Let $\mathscr{A}$ be the space of operator valued meromorphic functions and $\mathscr{K}$ the space of operator valued meromorphic differential forms. From the last section, we have an exterior derivative $d: \mathscr{A} \rightarrow \mathscr{K}$. We consider an operator valued differential form $A$ to be exact if it is of the form $A=d \alpha$ for some operator valued meromorphic function $\alpha$. We can now state our third axiom for operator valued differential forms:

(iii) If $A=d \alpha$, then $\operatorname{Res}_{P} A=0$ for all $P$.

\footnotetext{
${ }^{18}$ It is shown in the appendix to [19] that if one is willing to work on the moduli space of surfaces of given genus rather than a particular surface $\Sigma$, one can actually extend this to an action of the whole Virasoro Lie algebra $\hat{\mathscr{R}}$
} 


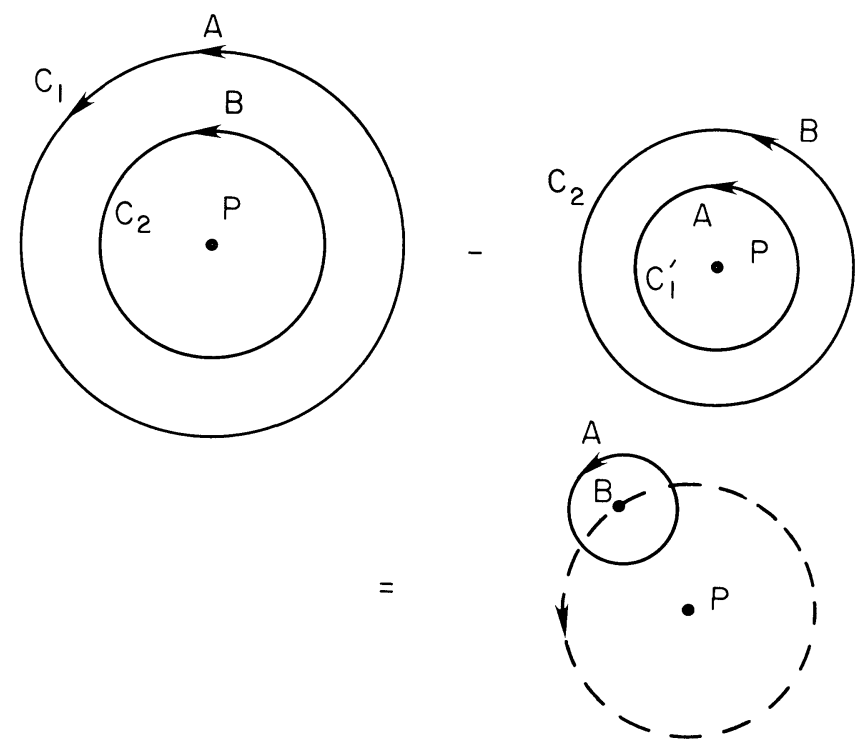

Fig. 7. A contour argument which is used to compute the commutator $\left[\operatorname{Res}_{P}(A), \operatorname{Res}_{P}(B)\right]$, with $A$ and $B$ being two operator valued differential forms

The justification for this is very simple. Picking a local parameter $z$ at a point $P \in \Sigma$, the formula $A=d \alpha$ just means $A=d \alpha / d z$. So the residue at $P$ is

$$
\operatorname{Res}_{P}(A)=\frac{1}{2 \pi i} \oint_{C} d z \frac{d \alpha}{d z}=0,
$$

this being just the statement that the integral of a total derivative around the closed contour $C$ is zero. This reasoning is indeed trivial; the only difficulty in the last section was to show that there is a geometrically defined object $d \alpha$ which upon picking a local coordinate $z$ reduces to $d \alpha / d z$.

Having associated to a point $P$ and an operator valued differential form $A$ an operator $\operatorname{Res}_{P}(A)$ in the Hilbert space $V_{P}$ at $P$, it is natural to try to compute the commutators of these operators. Thus, let $A$ and $B$ be two operator valued differential forms, and let us study the commutator of $\operatorname{Res}_{P}(A)$ and $\operatorname{Res}_{P}(B)$. This can be done by a contour deformation argument which is familiar in conformal field theory (Fig. 7). Let $C_{2}$ be a contour that encloses $P$, and $C_{1}$ a contour that encloses $C_{2}$, and such that $C_{1}$ and $C_{2}$ are small enough to enclose no poles of $A$ and $B$ except the possible poles at $P$. Then acting on some state $|\Lambda\rangle \in V_{P}$ we have

$$
\operatorname{Res}_{P}(A) \operatorname{Res}_{P}(B)|\Lambda\rangle=\left(\frac{1}{2 \pi i}\right)^{2} \oint_{C_{1}} A\left(z_{1}\right) \oint_{C_{2}} B\left(z_{2}\right)|\Lambda\rangle .
$$

Here $z_{1}$ and $z_{2}$ are parameters along $C_{1}$ and $C_{2}$. To take the two operators in opposite order, let $C_{1}^{\prime}$ be a contour inside $C_{2}$; we get

$$
\operatorname{Res}_{P}(B) \operatorname{Res}_{P}(A)|\Lambda\rangle=\left(\frac{1}{2 \pi i}\right)^{2} \oint_{C_{2}} B\left(z_{2}\right) \oint_{C_{1}^{\prime}} A\left(z_{1}\right)|\Lambda\rangle .
$$


We want to consider the difference $\left(\operatorname{Res}_{P}(A) \operatorname{Res}_{P}(B)-\operatorname{Res}_{P}(B) \operatorname{Res}_{P}(A)\right)|\Lambda\rangle$. This difference is of course a difference of double contour integrals. Doing the integral over $z_{1}$ first for fixed $z_{2}$, we see that the $z_{1}$ contour is $C_{1}-C_{1}^{\prime}$, a contour which encircles $z_{2}$ but not $P$. Thus, we define

$$
(A \circ B)\left(z_{2}\right)=\frac{1}{2 \pi i} \oint_{C} A(z) B\left(z_{2}\right),
$$

with $C$ any contour (such as $C_{1}-C_{1}^{\prime}$ ) that encircles $z_{2}$ and no other poles of $A$. Then

$$
\left[\operatorname{Res}_{P}(A), \operatorname{Res}_{P}(B)\right]|\Lambda\rangle=\frac{1}{2 \pi i} \oint_{C_{2}}(A \circ B)\left(z_{2}\right)|\Lambda\rangle .
$$

The right-hand side, however, is precisely the residue operation that we have been discussing, so we may state this more succinctly in the form

$$
\left[\operatorname{Res}_{P}(A), \operatorname{Res}_{P}(B)\right]=\operatorname{Res}_{P}(A \circ B) .
$$

This is the desired formula for commutators of operator valued residues. We can now state the fourth axiom for such residues.

(iv) Let $A$ and $B$ be operator valued differential forms. Let $Q$ be a point at which $B$ does not have a pole. Then the value of $B$ at $Q$ determines a state $B_{Q}$ in the space $V_{Q}$ of observables at $Q$. Let $(A \circ B)_{Q}=\operatorname{Res}_{P}(A) \cdot B_{Q}$. Then there is a meromorphic operator valued differential form $A \circ B$ which equals $(A \circ B)_{Q}$ except perhaps at finitely many $Q$ where $A$ or $B$ has a pole. Moreover $\left[\operatorname{Res}_{P}(A), \operatorname{Res}_{P}(B)\right]=\operatorname{Res}_{P}(A \circ B)$.

[In the above, the reason for avoiding poles of $A$ and $B$ is that $B_{Q}$ is not defined at a pole of $B$, and the $\operatorname{Res}_{P}(A)$ operation can be seen to depend meromorphically on $P$ only if one keeps away from poles of $A$.]

Now, $A \circ B$ can be computed, say, from path integrals with insertions of

$$
\oint_{C} A(z) \cdot B(w),
$$

with $C$ a contour circling $w$. In this formula, $B$ plays a relatively passive role. It is obvious, for instance, that if $f$ is a meromorphic function, then $A \circ(f B)=f(A \circ B)$. It is also clear that (127) still makes sense if $B$ is replaced by an operator valued meromorphic function $\phi$ rather than an operator valued differential form. We would simply study insertions of

$$
\oint_{C} A(z) \cdot \phi(w)
$$

to compute $A \circ \phi$. For operator valued functions we have a natural exterior derivative $\phi \rightarrow d \phi$. Extracting this derivative clearly commutes with the operation of multiplying by $\oint_{C} A(z)$, so $A \circ(d \phi)=d(A \circ \phi)$. Likewise, the operation in (128) clearly commutes with multiplying $\phi$ by a $c$-number function or taking its tensor product with a $c$-number differential form. We can thus add the following to our list of axioms:

(v) For A an operator valued differential form and $\phi$ an operator valued function, there is an operator valued function $A \circ \phi$ such that for all but finitely many $Q$ where $A$ or $\phi$ has a pole, $(A \circ \phi)_{Q}=\operatorname{Res}_{Q}(A) \cdot \phi_{Q}$. This operation is compatible with the 
operation $A \circ B$ for differential forms $A, B$, in the sense that if $\omega$ is a c-number meromorphic differential form, so that $\phi \otimes \omega$ is an operator valued differential form, then

$$
(A \circ \phi) \otimes \omega=A \circ(\phi \otimes \omega) .
$$

(On the left of (129) there appears the composition of a differential form with a function, and on the right there appears the composition of two differential forms.) If moreover $f$ is a meromorphic function, then $A \circ(f \phi)=f(A \circ \phi)$. And finally, the 。 operation commutes with the exterior derivative in the sense that

$$
A \circ(d \phi)=d(A \circ \phi) \text {. }
$$

We will devote the rest of this section to discussing certain facts which follow from peculiarities of (126). The left-hand side is antisymmetric in $A$ and $B$, but the right-hand side is not manifestly antisymmetric. It follows from (126) that $A \circ B+B \circ A$ must have zero residues. This does not mean that $A \circ B+B \circ A$ is zero. We have learned that there is a large class of operator valued differential forms with zero residues, namely the exact forms. Computation in simple examples shows that $A \circ B+B \circ A$ need not be zero, but is an exact form. For instance, let $\chi$ and $\psi$ be two free fermions, with propagator $\langle\psi(z) \psi(w)\rangle=\langle\chi(z) \chi(w)\rangle=1 /(z-w)$. Let $A(z)=\chi d \psi(w)$ and $B(w)=\psi(w)$. To compute $A \circ B$ we take the operator product

$$
A(z) B(w)=\sum_{n}(z-w)^{n} O_{n}(w),
$$

and then $A \circ B(w)=O_{-1}$. The singular part of $A(z) B(w)$ is

$$
-\frac{\chi(z)}{(z-w)^{2}}=-\frac{\chi(w)}{(z-w)^{2}}-\frac{d \chi(w)}{z-w}+\ldots
$$

Evidently, $O_{-1}=-d \chi$, so

$$
A \circ B(w)=-d \chi(w) .
$$

On the other hand, to compute $B \circ A$, we express the same operator product in an expansion around $z$ :

$$
A(z) B(w)=\sum_{n} \frac{O_{n}^{\prime}}{(w-z)^{n}} .
$$

Then $B \circ A=O_{-1}^{\prime}$. But clearly

$$
B(w) A(z)=-\frac{\chi(z)}{(w-z)^{2}}+\ldots
$$

There is no single pole, so $B \circ A=0$. Therefore, in this example,

$$
A \circ B+B \circ A=-d \chi,
$$

and as expected the differential form on the right-hand side is exact (being $d$ of the operator valued function $\chi$ ). This then illustrates the general rule which is needed for the consistency of (126):

(vi) Let $A$ and $B$ be operator valued differential forms. Then $A \circ B+B \circ A$ is an exact form, say $A \circ B+B \circ A=d K(A, B)$, where $K(A, B)$ (defined up to an additive $c$ number constant) is an operator valued meromorphic function depending on $A$ and $B$. 


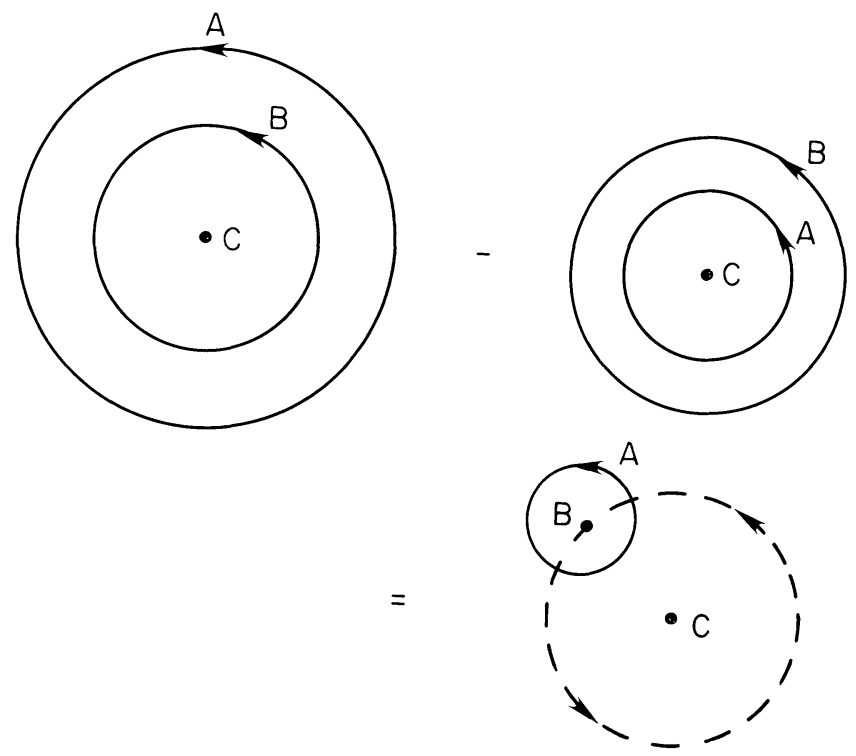

Fig. 8. Contour integrals for three operator valued differential forms $A, B$, and $C$

The exactness of $A \circ B+B \circ A$ has a counterpart, in a somewhat different context, in recent work by Strominger on string field theory [22]. The relationship deserves to be explored more fully.

Finally, one more aspect of the consistency of (126) needs to be investigated. The equation $\left[\operatorname{Res}_{P}(A), \operatorname{Res}_{P}(B)\right]=\operatorname{Res}_{P}(A \circ B)$ suggests that under the $\circ$ operation, operator valued differential forms (or at least such forms modulo exact ones) must form a Lie algebra. Let us try to understand the Jacobi identity.

In Fig. 8 we consider contour integrals for composition of three operator valued differential forms $A, B$, and $C$. From the arrangement of contours in the figure, one can see that there is a Jacobi-like identity

$$
A \circ(B \circ C)-B \circ(A \circ C)=(A \circ B) \circ C .
$$

Equation (137) is not quite a Jacobi identity; in fact, the o operation cannot possibly be a Lie bracket, since it is not even antisymmetric. Even if we define a new operation $[$,$] by [A, B]=(A \circ B-B \circ A) / 2$, (137) does not become a Jacobi identify for $[$,$] . To get a Lie algebra from (137), it is necessary to work not with$ operator valued differential forms $\mathscr{K}$, but with the quotient $\mathscr{K} / d \mathscr{A}$ of operator valued differential forms by exact ones. From our above axioms, we have

$$
(A+d u) \circ B=A \circ B, \quad A \circ(B+d u)=A \circ B+d(A \circ u) .
$$

Let us denote the equivalence class of a differential form $A$ in $\mathscr{K} / d \mathscr{A}$ as $\bar{A}$. From (138) it follows that if $\bar{A}_{1}=\bar{A}_{2}$, and $\bar{B}_{1}=\bar{B}_{2}$, then

$$
\overline{\left(A_{1} \circ B_{1}\right)}=\overline{\left.A_{2} \circ B_{2}\right)} \text {. }
$$


This meeans that the o operation on $\mathscr{A}$ induces a well defined operation $\bar{A}, \bar{B}$ $\rightarrow \bar{A} \circ \bar{B}$ on $\mathscr{K} / d \mathscr{A}$. Since the o operation in $\mathscr{K} / d \mathscr{A}$ is automatically antisymmetric, we denote it as $[$,$] .$

Now, although (137) is not and cannot be a Jacobi identity for a $\circ$ or $[$, operation in $\mathscr{K}$, it is easy to see that (137) induces the Jacobi identity for the $[$, operation in $\mathscr{K} / d \mathscr{A}$. Therefore, $\mathscr{K} / d \mathscr{A}$ has a natural Lie algebra structure.

What is the center of this Lie algebra? In the vertex operator algebra of a conformal field theory, the center is the identity operator 1, the vertex operator for coupling to the vacuum state. In a general operator valued differential form $A=\sum a_{i} \psi^{i}$, one of the operators $\psi^{i}$, say $\psi^{0}$, is the identity operator, and the corresponding coefficient function $a_{0}$ is an ordinary $c$-number differential form. Since their "operator" part is the identity operator, such $c$-number differential forms are in the center of $\mathscr{K} / d \mathscr{A}$.

Let $\Omega^{1}$ be the space of $c$-number meromorphic differential forms on the compact Riemann surface $\Sigma$ under study. Before identifying the center of $\mathscr{K} / d \mathscr{A}$ with $\Omega^{1}$, we must take the quotient of $\Omega^{1}$ by the exact differential forms, since exact forms are equivalent to zero in $\mathscr{K} / d \mathscr{A}$. Thus, let $\Omega^{0}$ be the space of $c$-number meromorphic functions on $\Sigma$. It is the quotient $\Omega^{1} / d \Omega^{0}$ which will be the center of $\mathscr{K} / d \mathscr{A}$.

(vii) The o operation on $\mathscr{K}$ induces a Lie algebra structure on $\mathscr{K} / d \mathscr{A}$, and the center of $\mathscr{K} / d \mathscr{A}$ is $\Omega^{1}(\Sigma) / d \Omega^{0}(\Sigma)$.

If $\mathscr{U}$ is the quotient of $\mathscr{K} / d \mathscr{A}$ by its center, then $\mathscr{K} / d \mathscr{A}$ is a central extension of $\mathscr{U}$ by $\Omega^{1} / d \Omega^{0}(\Sigma)$ :

$$
0 \rightarrow \Omega^{1} / d \Omega^{0} \rightarrow \mathscr{K} / d \mathscr{A} \rightarrow \mathscr{U} \rightarrow 0 .
$$

A central extension of the Lie algebra of meromorphic vector fields by $\Omega^{1} / d \Omega^{0}$ was formulated in [19]. What we have described here is essentially the physical setting for that construction, as well as a generalization to the whole operator algebra of a conformal field theory. If one considers only the two operators 1 and $T$ (the identity and the energy-momentum tensor), then $\mathscr{U}$ becomes the Lie algebra of meromorphic vector fields, and (140) is the extension constructed in [19]. This example is worked out explicitly in the appendix.

\section{Quantum Field Theory on an Algebraic Curve}

A traditional and powerful way to construct a quantum field theory is to find a Lagrangian and then "quantize." In the case of quantum field theory on a Riemann surface, this method has two deficiencies. First (unlike the situation for quantum field theory in higher dimensions), there are many conformal quantum field theories on Riemann surfaces for which a suitable Lagrangian apparently does not exist. In fact, among holomorphic quantum field theories on a Riemann surface, the theory of free fermions is perhaps the only case in which one knows of a really satisfactory Lagrangian.

Second, it is very tempting to believe that one should learn to formulate conformal quantum field theory not just on a Riemann surface over the complex numbers $\mathscr{C}$, but on a curve over an arbitrary ground field $k$. While one would wish to have an analogue of Lagrangians and quantization of Lagrangians in this more general setting, such notions appear rather distant at present. 
When a Lagrangian is not available, one can attempt to describe quantum field theory in terms of Ward identities, an approach which in the case of conformal quantum field theory on Riemann surfaces was shown in [14] to be particularly powerful. The Ward identity approach is basically concerned with operator valued residues of operator valued differential forms, whose basic properties were described in the last section. Ordinary residues of ordinary differential forms make sense in an algebraic setting. This encourages us to believe that operator valued residues of operator valued differential forms - and thus the Ward identity approach to quantum field theory - can make sense over an arbitrary ground field $k$. The purpose of this section is to work this out for some of the simplest conformal quantum field theories.

We consider first the case of free fermions. We work on a smooth complete curve $X$ over an algebraically closed ground field $k$. (For convenience, we take the characteristic of $k$ not equal to two; otherwise, one must replace Clifford algebras by quadratic forms.) Let $L$ be the canonical line bundle of $X$ and let $L^{1 / 2}$ denote a chosen square root of $L$.

The heuristic idea behind the construction has to do with ideas described in the last section. The fermion field $\psi$ is on "operator-valued section of $L^{1 / 2}$." If then $f$ is a rational section of $L^{1 / 2}$, the product $w_{f}=f \psi$ is an "operator valued differential form." We will then define the "operator valued residue" of $w_{f}$, and require that "the sum of the operator valued residues is zero." The latter statement was one of the properties of operator valued differential forms formulated in the last section. Having already explained the physical ideas in the last section, we will here simply proceed with the mathematical constructions, presented hopefully in a selfcontained way.

Let $Y$ be the space of rational sections of $L^{1 / 2}$. It is an infinite dimensional vector space over $k$. Let $\wedge Y$ be the exterior algebra on $Y$. As a vector space it is

$$
\wedge Y=1 \oplus Y \oplus \wedge^{2} Y \oplus \ldots
$$

with $\wedge{ }^{k} Y$ the $k^{\text {th }}$ exterior power. (The symbol "1" stands for a one dimensional vector space.)

Let us recall the notion of a Clifford algebra. Given a $k$ vector space $V$ with a nondegenerate quadratic form (, ), one defines a clifford algebra $C V$ as follows. Elements $v, w$ of $V$, regarded as elements of $\wedge V$, anticommute,

$$
v \wedge w+w \wedge v=0 .
$$

In the Clifford algebra, (143) is replaced by the Clifford multiplication law

$$
\hat{v} \hat{w}+\hat{w} \hat{v}=-2(v, w) c .
$$

(Here $c$ is the one dimensional center of the Clifford algebra. One may suppress it and set $c=1$.) Here $v \rightarrow \hat{v}$ can be regarded as a natural "lift" of $V \subset \wedge V$ to $C V$; this lift does not preserve the Grassmann algebra (143), the correction being the cocycle which appears on the right-hand side of (144) and gives the Clifford algebra $C V$ as a deformation of the Grassmann or exterior algebra $\wedge V$. 
Now, on the space $Y$ of rational sections of $L^{1 / 2}$, there is no natural quadratic form, and thus no natural way to make a Clifford algebra. However, let $P$ be a point in $X$. For $f, g$ in $Y$, the product $f g$ is a differential form. Let $\operatorname{Res}_{P}$ denote the operation of taking the residue of a differential form at $P$. The formula

$$
(f, g)_{P}=\operatorname{Res}_{P}(f g)
$$

then defines a quadratic form $(,)_{P}$ on $Y$.

In fact, let $Y_{P}$ be the completion of $Y$ at $P$, or in other words the space of formal sections of $L^{1 / 2}$ defined by a formal power series near $P$. Upon trivializing $L^{1 / 2}$ in a neighborhood $P$, an element of $Y_{P}$ can be expressed as a formal series

$$
\sum_{n=-N}^{\infty} a_{n} z^{n}
$$

with $z$ a uniformizer at $P$ and $a_{n} \in k$. Since $Y$ has a natural embedding in its completion $Y_{P}$, the "global exterior algebra" $\wedge Y$ has a natural embedding in $\wedge Y_{P}$ for each $P$. Different completions $Y_{P}$ and $Y_{Q}$ of $Y$ for $P \neq Q$ are very different spaces, since there is no way to reexpress a formal series at $P^{19}$ as a series at $Q$. The quadratic form $(,)_{P}$ makes sense not just on $Y$ but on its completion $Y_{P}$, since it makes sense to compute the residue at $P$ of a differential form which is defined just in a formal power series at $P$. (For $Q \neq P$, the quadratic form $(,)_{Q}$ cannot be defined in $Y_{P}$, since there is no way to reexpand the formal series (146) around $Q$.) The Clifford algebra constructed from $Y_{P}$ with the quadratic form $(,)_{P}($ and $c=1)$ will be called $C Y_{P}$.

We next wish to construct a representation of $C Y_{P}$ on a $k$-vector space $V_{P}$ which in physical terminology is "the space of observables at $P$." The irreducible representation of a finite dimensional Clifford algebra is unique. This is not true in infinite dimensions. To specify a representation of $C Y_{P}$ requires a choice of "polarization" of $(,)_{P}$ - that is, a choice of a maximal subspace $Y_{P}^{+}$of $Y_{P}$ such that $(f, g)_{P}=0$ for $f, g \in Y_{P}^{+}$. For $Y_{P}^{+}$we choose the space of sections of $L^{1 / 2}$ (or rather, formal series (146)) which are regular at $P$. Up to isomorphism, there is a unique irreducible representation $V_{P}$ of $C Y_{P}$ which contains a vector $1_{P}$ annihilated by $Y_{P}^{+}$ (or rather the lift of $Y_{P}^{+}$to the Clifford algebra).

A standard description of $V_{P}$ would be the following. Pick a complement $Y_{P}^{-}$to $Y_{P}^{+}$in $Y_{P}$. A standard and convenient way to pick such a complement is to trivialize $L^{1 / 2}$ in a neighborhood of $P$, pick a uniformizer $z$ at $P$, and then take $Y_{P}^{-}$to consist of "polar" series $\sum_{n=1}^{N} a_{n} z^{-n}$. Then as a vector space one defines $V_{P}$ by the formula

$$
V_{P}=\wedge Y_{P}^{-}=1_{P} \oplus Y_{P}^{-} \oplus \wedge^{2} Y_{P}^{-} \oplus \ldots
$$

Thus, $V_{P}$ is the sum of exterior powers of $Y_{P}^{-} \cdot\left[1_{P}\right.$, whose analogue in Eq. (141) was simply called " 1 ," denotes a one dimensional vector space with basis element $1_{P}$.] Thus, $V_{P}$ has a basis consisting of elements of the form

$$
v=f_{1} \wedge f_{2} \wedge \ldots \wedge f_{k}
$$

\footnotetext{
${ }^{19}$ Which is not necessarily a convergent series, even if we are working over $\mathscr{C}$ where there would be a notion of convergence
} 
with $f_{m} \in Y_{P}^{-}$and $k=0,1,2, \ldots$ For $f \in Y_{P}$, let $f=f^{+}+f^{-}$, with $f^{ \pm} \in Y_{p}^{ \pm}$. The action of $f$, or rather its lift $\hat{f}$ to $C Y_{P}$, on the vector $v$ of (148) is then defined to be

$$
\hat{f} \cdot v=f^{-} \wedge v-2 \sum_{m=1}^{k}(-1)^{m}\left(\operatorname{Res}_{P}\left(f^{+} f_{m}\right)\right) \cdot f_{1} \wedge \ldots \wedge \hat{f}_{m} \wedge \ldots \wedge f_{k} .
$$

(On the right the symbol $\hat{f}_{m}$ means that $f_{m}$ is to be omitted.) This formula gives a representation of $C Y_{P}$ which up to isomorphism is independent of the choice of $Y_{P}^{-}$. While (149) may look cumbersome, it is actually the perfectly standard formula for the action of the Clifford algebra on a fermion Fock space.

In the preceding, $V_{P}$ is what usually would be called "the space of quantum field theory observables at $P$." We now wish to consider the space of "all observables on $X$." In quantum field theory, an observable is usually a finite product $\prod_{i=1}^{N} O_{i}\left(P_{i}\right)$ of observables at points $P_{i}$, or alternatively a product $\prod_{P} O(P)$ which runs over all $P \in X$ with the restriction that $O(P)=1$ for all but finitely many $P$. Thus, the appropriate object is the restricted or adelic product of the $V_{P}$,

$$
V=\coprod_{P} V_{P} .
$$

An element of $V$ is an element $\otimes_{P} v_{P}$ of the ordinary tensor product $\otimes_{P} V_{P}$ such that $v_{P}=1_{P}$ for all but finitely many $P$. (The bizarre-looking symbol $\amalg$ means nothing more nor less than a "restricted" tensor product, restricted by the condition just stated that almost all $v_{P}$ equal $1_{P}$. We will often later use this symbol $\amalg$ to denote similar although slightly different restricted infinite products.) The special vector $1=\otimes_{P} 1_{P}$ is called the "vacuum" in physical discussions. A restricted product $V=\coprod_{P} V_{P}$ of local vector spaces $V_{P}$, one for each point $P$ on a curve, is a standard notion in the theory of automorphic representations [7], and one of our main points in this section and the next is to translate some physical concepts into the terminology of automorphic representation theory.

Each Clifford algebra $C Y_{P}$ acts on $V$ in a natural way. We just let $C Y_{P}$ act on the $V_{P}$ component in $V=\coprod_{Q} V_{Q}$ according to the product law (149), while leaving invariant the $V_{Q}$ components of $Q \neq P$. Thus, if $v \in V$ is $v=\otimes_{Q} v_{Q}$, then for $y_{P} \in C Y_{P}$, we set

$$
y_{P} \cdot v=\underset{Q}{\otimes} v_{Q}^{\prime},
$$

where $v_{Q}^{\prime}=v_{Q}$ for $Q \neq P$ and $v_{P}^{\prime}=y_{P} \cdot v_{P}$.

We consider an element $y_{P}$ of $C Y_{P}$ to be "regular at $P$ " if it is constructed as in (148) from a wedge product of sections of $L^{1 / 2}$ that are regular at $P$. We wish to define a sort of adelic product

$$
C Y=\coprod_{P} C Y_{P}
$$

of the individual Clifford algebras $C Y_{P}$. The adelic product $\coprod_{P} C Y_{P}$ is defined as follows. An element of $C Y$ is defined to be a formal sum $\oplus_{P} y_{P}$, with $y_{P}$ in $C Y_{P}$ for all $P$, and $y_{P}$ regular at $P$ for all but finitely many $P$. Also, in forming the restricted product of the $C Y_{P}$, we identify their central elements, so that $C Y$ has only a one 
dimensional center generated by an object $c^{20}$. Likewise, we define the restricted product $\coprod_{P} \wedge Y_{P}$ to consist of objects $\oplus_{P} y_{P}$ with each $y_{P} \in \wedge Y_{P}$ and almost all $y_{P}$ regular at $P$.

The motivation for the restriction in the definition of the global algebra $C Y$ is to ensure that $C Y$ can act on the global space of observables $V$ in a natural way. The point is that if $y_{P}$ is regular at $P$, it is a sum of products of annihilation operators [since in (149) $f$ behaves as an annihilation operator if it is regular at $P$, that is if $\left.f^{-}=0\right]$. Hence, if $y_{P}$ is regular at $P$, it annihilates the vacuum vector $1_{P}$. This permits us to define an action of $C Y$ on $V$ as follows. For $y=\oplus_{P} y_{P} \in C Y$ and $v=\otimes_{P} v_{P} \in V$, we define

$$
y \cdot v=\sum_{P} y_{P} \cdot v
$$

with $y_{P} \cdot v$ as already defined in Eq. (151). The definition makes sense because $y_{P} \cdot v=0$ for all but finitely many $P$ (since $v_{P}=1_{P}$ for all but finitely many $P$, and $y_{P}$ is regular at $P$ for all but finitely many $P$ ).

Recall now that each $C Y_{P}$ is a deformation of $\wedge Y_{P}$. For each $P, Y \subset Y_{P} \subset \wedge Y_{P}$ has a natural lift to $C Y_{P}$. This lifting does not preserve the exterior algebra. Rather, for $f, g \in Y$, the lifts $\hat{f}_{P}, \hat{g}_{P}$ do not anticommute, but obey the Clifford relations

$$
\hat{f}_{P} \hat{g}_{P}+\hat{g}_{P} \hat{f}_{P}=-2 c \operatorname{Res}_{P}(f g)
$$

To recapitulate what we have done so far, we began with a Grassmann algebra $\wedge Y$. Clifford algebras are such a natural generalization of Grassman algebras that we were tempted to try to find a Grassmann algebras as a deformation of $\wedge Y$. Doing so requires a quadratic form on $Y$, and there is no natural global choice. However, picking $P \in X$, there is a natural "local" quadratic form $(,)_{P}$ at $P$, and using it we constructed a "local deformation of $\wedge Y$ at $P$," namely $C Y_{P}$. Since we wish to work globally, we then combine the $C Y_{P}$ into a global object $C Y$. This object is significant because on the one hand it is the Clifford algebra most naturally associated with the global curve $X$, and on the other hand its irreducible Clifford module $V$ is "the space of observables of the free fermion quantum field theory."

The next key element is the following. $\wedge Y$ has a natural embedding in each of its completions $\wedge Y_{P}$. Therefore, given $f \in \wedge Y$, we can naturally regard $f$ as an element of $\wedge Y_{P}$ for any $P$. Putting these together, we get the "diagonal embedding" of $\wedge Y$ in $\coprod_{P} \wedge Y_{P}$,

$$
\Delta: \wedge Y \rightarrow \coprod_{P} \wedge Y_{P}
$$

defined as follows. Given $f \in \wedge Y, \Delta(f) \in \coprod_{P} \wedge Y_{P}$ is the object $\oplus_{P} f_{P}$, where $f_{P}=f$ for all $P$. This makes sense because any $f \in \wedge Y$ is regular at $P$ for all but finitely many $P$. Since $Y$ is embedded in $\wedge Y$, this gives us in particular a diagonal embedding of $Y$ in $\coprod_{P} \wedge Y_{P}$.

${ }^{20}$ The latter remark is of course unnecessary if the reader has mentally set $c=1$ from the beginning 
We want to interpret global rational sections of $L^{1 / 2}$, that is, elements of $Y$, as operators on the space $V$ of observables. To this end, the diagonal embedding (157) of $Y \subset \wedge Y$ in $\coprod_{P} \wedge Y_{P}$ is not good enough. For it is the Clifford algebra $C Y$, not the exterior algebra $\coprod_{P} \wedge Y_{P}$ that has a natural action on $V$. We must lift $Y$ from $\wedge Y$ to $C Y$.

We already discussed in (155) a natural lift $f \rightarrow \hat{f}_{P}$ of $Y \subset \wedge Y$ to $C Y_{P}$. Adding these componentwise, we get a natural lift

$$
f \rightarrow \hat{f}=\sum_{P} \hat{f}_{P}
$$

of $Y$ to $C Y$. We now see for $f, g \in Y$ by summing (155) over $P$ that

$$
\hat{f} \hat{g}+\hat{g} \hat{f}=-2 c \sum_{P} \operatorname{Res}_{P}(f g)=0 .
$$

Here we have used the fact that the sum of the residues of a differential form is zero. Equation (159) is one of the key equations in our present discussion. It means that the global exterior algebra $\wedge Y$ with which we began can be embedded in the adelic Clifford algebra $C Y$ while preserving the commutation relations. [It cannot be embedded in any $(C Y)_{P}$ because of the Clifford relations.

More generally, let $\wedge Y^{+}=Y \oplus \wedge^{2} Y \oplus \ldots$ be the sum of the positive exterior powers of $Y$. Since $\wedge Y^{+}$is generated as an algebra by $Y$, the embedding $Y \rightarrow C Y$ extends to an embedding of $\wedge Y^{+}$in $C Y$ - which preserves the exterior multiplication in $\wedge Y^{+}$in view of (159). For $f \in \wedge Y^{+}$we denote the lift to $C Y$ as $\hat{f}$.

We now wish to describe the "expectation value of a product of observables." This is a linear functional on $V$, which we will call $\int: V \rightarrow k$. The functional is required to obey

$$
\int \hat{f} \cdot v=0
$$

for all $v \in V$ and $f \in Y$. Of course, since $\wedge Y^{+}$is generated by $Y$ as an algebra, this is the same as saying

$$
\int \hat{y} \cdot v=0
$$

for all $y \in \wedge Y^{+}, v \in V$. Equation (160) or (161) is the "Ward identity of free fermion field theory" and is analogous to the statement that the sum of the residues of a differential form is zero. The rationale for such formulas in quantum field theory was discussed in Sect. (3).

It is important to observe that the possibility of imposing the Ward identities (160) depends on the validity of (159). If we had $y, z \in Y$ with $\{\hat{y}, \hat{z}\}$ nonzero, and equal, say to a non-zero element $\alpha$ of the ground field $k$, then (160) would imply

$$
0=\int\{\hat{y}, \hat{z}\} \cdot v=\alpha \cdot \int v
$$

and therefore the operation $\int$ would have to vanish. The global formula (159) is necessary for the Ward identities to make sense. ${ }^{21}$

\footnotetext{
${ }^{21}$ It may be that in trying to go "off shell" so as to formulate string theory geometrically, instead of considering non-conformally invariant field theories one should consider conformally invariant structures that are not field theories because some central extension does not split globally
} 
To understand the content of (160) or (161), a key fact is the following. Let $\left(\wedge Y^{+}\right) V$ be the subspace of $V$ consisting of expressions $\sum \hat{y}_{i} \cdot v_{i}$, with $y_{i} \in \wedge Y^{+}$, $v_{i} \in V$. Then the quotient space $V /\left(\wedge Y^{+}\right) V$ is one dimensional. As a result, a linear map $\int: V \rightarrow k$ obeying (160) exists and is unique up to multiplication by a scalar.

In showing that $V /\left(\wedge Y^{+}\right) V$ is one dimensional, ${ }^{22}$ we will for simplicity (though it is not necessary) assume that the chosen square root $L^{1 / 2}$ of $L$, which we have kept fixed in this discussion, has no global sections, i.e., $H^{0}(L)=0$.

Consider in $V_{P}$ a general monomial $w=f_{1} \wedge f_{2} \wedge \ldots \wedge f_{k}$. Each $f_{r}$ has a pole at $P$, say or order $n_{r}$, because of the way $V_{P}$ is constructed [recall Eq. (147)]. We define an integer $|w|$ by writing $|w|=\sum_{r} n_{r}$. A general vector in $V_{P}$ is $u=\sum \alpha_{i} w_{i}$ with $\alpha_{i} \in k$ and $w_{i}$ a monomial. We say $u$ is of order $|u|=\max \left|w_{i}\right|$. If $v \in V$ is $v=\otimes_{P} v_{P}$, we write $|v|=\sum_{P}\left|v_{P}\right|$. Thus $|v|=0$ if and only if $v=\otimes_{P} 1_{P}$ is the vacuum vector. Otherwise $|v|>0$. For $v \in V$ we write $\bar{v}$ for the image of $V$ in $V /\left(\wedge Y^{+}\right) V$. We will show that if $|v|>0$, there is $v^{\prime} \in V$ with $\left|v^{\prime}\right|<|v|$ and $\bar{v}^{\prime}=\bar{v}$. Applied repeatedly, this will show that for every $v \in V$ there is $\alpha \in k$ with $\bar{v}=\alpha \cdot \overline{1}, 1 \in V$ being the "vacuum" $1=\otimes_{P} 1_{p}$.

Given $v$ with $|v|>0$, to find $v^{\prime}$ with $\bar{v}=\bar{v}^{\prime}$ and $\left|v^{\prime}\right|<|v|$ we proceed as follows. Let $v=\otimes_{p} v_{p}$. It clearly suffices to consider the case with each $v_{p}$ a monomial, since any $v$ is a linear combination of these. Pick $P$ with $\left|v_{P}\right|>0$, say $v_{P}=f_{1} \wedge f_{2} \ldots \wedge f_{k}$. Define $u=\otimes_{Q} u_{Q}$, where $u_{Q}=v_{Q}$ for $Q \neq P$ and $u_{P}=f_{2} \wedge \ldots \wedge f_{k}$. Let $g$ be a global rational section of $L^{1 / 2}$ such that $g$ is regular except at $P$ and $g-f_{1}$ is regular at $P$. Such a $g$ exists by the Riemann-Roch theorem [for $H^{0}\left(L^{1 / 2}\right)=0$ as we assume]. Then $v^{\prime}=v-\hat{g} u$ has the required properties $\left|v^{\prime}\right|<|v|$ and $\bar{v}^{\prime}=\bar{v}$. The reason for this is as follows: in acting on $u, \hat{g}$ can behave as a creation operator only on the $P$ component, this being possible because of the pole in $g$ at $P$. Acting at $P$ as a creation operator, $\hat{g}$ turns $u$ into $v$. Otherwise, $\hat{g}$ acts as an annihilation operator, lowering $|u|$, which is already less than $|v|$. Hence $|v-\hat{g} \cdot u|<|v|$.

Repeating this process eventually gives an expression $v=\alpha \cdot 1+\hat{y} \cdot 1$ with $y \in \wedge Y^{+}$. This shows that $V /\left(\wedge Y^{+}\right) V$ is at most one dimensional. To show that $V /\left(\wedge Y^{+}\right) V$ is in fact one dimensional, we must show that $\overline{1} \neq 0$, i.e. that there is no relation $1=\Sigma y_{i} \cdot v_{i}$. Since each $v_{i}$ can (as we have just shown) be written in the form $v_{i}=\alpha_{i} \cdot 1+\hat{y}_{i} \cdot 1$, it is enough to show that a relation $\beta \cdot 1=\hat{y} \cdot 1$, with $\beta \in k$, $y \in(\wedge Y)^{+}$, implies $\beta=y=0$. This is straightforwardly proved somewhat along the lines of the above. For $f \in Y$, let $|f|$ be the number of poles of $f$ counted by multiplicity. If $\alpha \in(\wedge Y)^{+}$can be written as $f_{1} \wedge f_{2} \wedge \ldots \wedge f_{k}$ we would like to define $|\alpha|$ as $\Sigma\left|f_{i}\right|$. We must be careful here since the representation $\alpha=f_{1} \wedge \ldots \wedge f_{k}$, if it exists, is not unique [e.g. $f_{1} \wedge f_{2}=f_{1} \wedge\left(f_{2}-f_{1}\right)$ ]. To avoid such ambiguity, we pick a basis for $Y$. For each $P$ and each $n=1,2,3 \ldots$, let $f_{P, n}$ be an element of $Y$ (a section of $L^{1 / 2}$ ) regular except at $P$, and with a pole precisely of order $n$ at $P$. From the Riemann-Roch theorem, it follows that the $f_{P, n}$ exist and are a basis for $Y$. Then we consider only monomials of the form $y=f_{1} \wedge \ldots \wedge f_{k}$ with each $f_{i}$ one of the $f_{P, n}$. We define $|y|=\Sigma\left|f_{i}\right|$. It follows immediately from the definitions that the vector $\hat{y} \cdot 1$

${ }^{22}$ This statement is no more and no less than the statement that the Ward identities of fermion free field theory determine the correlation functions uniquely, and what follows is an essentially standard argument expressed so as to make sense over any ground field 
has $|\hat{y} \cdot 1|=|y|$, so a nontrivial representation $\beta \cdot 1=\hat{y} \cdot 1$ is impossible. More generally, if $y=\sum \alpha_{i} y_{i}$, with $\alpha_{i} \in k$ and $y_{i}$ a monomial of the form just considered, let $|y|=\max \left|y_{i}\right|$. Then again $|\hat{y} \cdot 1|=|y|$, completing the proof that $\overline{1} \neq 0$ and thus that the expectation value $\int: V \rightarrow k$ obeying (160) exists and is unique up to normalization. For $v \in V, \int v$ can be identified as $\bar{v}$, the image of $v$ in the one dimensional space $V /(\wedge Y)^{+} V$.

Since this description of free fermions may appear exotic, we will pause briefly to describe how to recover standard formulas. For each $P$, let $f_{P}$ be a section of $L^{1 / 2}$ regular except for a single pole at $P$. By the Riemann-Roch theorem, $f_{P}$ exists and is unique up to a scalar multiple. Given $n$ points $P_{1} \ldots P_{n}$, let $v_{P_{1} \ldots P_{n}} \in V$ be the vector $v=\otimes v_{P}$, where $v_{P}=f_{P}$ if $P$ is one of the $P_{i}$, and otherwise $v_{P}=1_{P}$. What in quantum field theory is usually called $\left\langle\psi\left(P_{1}\right) \ldots \psi\left(P_{n}\right)\right\rangle$ (in a theory of one component chiral fermions) is in our terminology here $\int v_{P_{1} \ldots P_{n}}$ or simply $\bar{v}_{P_{1} \ldots P_{n}}$. To see this explicitly for $n=2$, we compute $\bar{v}_{P_{1} P_{2}}$. Let $w=\otimes w_{P}$ with $w_{P}=1$ for $P \neq P_{2}$ and $w_{P_{2}}=f_{P_{2}}$. Then one computes

$$
\hat{f}_{P_{1}} w=v_{P_{1} P_{2}}-2 c \cdot \operatorname{Res}_{P_{2}} f_{P_{1}} f_{P_{2}} .
$$

Hence $\bar{v}_{P_{1} P_{2}}=\overline{1} \cdot 2 c \operatorname{Res}_{P_{2}}\left(f_{P_{1}} f_{P_{2}}\right)$. Because of the dependence on the choice of $f_{P_{1}}$ and $f_{P_{2}}, \operatorname{Res}_{P_{2}}\left(f_{P_{1}} f_{P_{2}}\right)$ is really a section of $L^{1 / 2} \otimes L^{1 / 2}$ over $\Sigma \times \Sigma$ rather than a function. Evidently, this section has a single pole at $P_{1}=P_{2}$ and is otherwise regular; this is the defining property of the Dirac propagator $G\left(P_{1}, P_{2}\right)$, as discussed in Sect. 1. Thus we have retrieved the Dirac propagator from the adelic description of free fermion quantum field theory.

This completes our discussion of free fermions on an algebraic curve. We would now like to discuss what one might call "current algebra on an algebraic curve." The discussion will be quite brief since it is analogous to what we have just described.

Let $\mathscr{U}$ be a finite dimensional simple Lie algebra over $k$ with a Killing form $\langle$,$\rangle . Let \mathscr{G}$ be the Lie algebra of rational maps of $X$ into $\mathscr{U}$. A cocycle for a central extension of $\mathscr{G}$ by $k$ would be a $k$ valued skew form $\phi\left(\lambda_{1}, \lambda_{2}\right)$ on $\mathscr{G}$ with

$$
\phi\left(\lambda_{1},\left[\lambda_{2}, \lambda_{3}\right]\right)+\phi\left(\lambda_{2},\left[\lambda_{3}, \lambda_{1}\right]\right)+\phi\left(\lambda_{3},\left[\lambda_{1}, \lambda_{2}\right]\right)=0 .
$$

This equation is the appropriate one to permit the existence of a central extension of Lie algebras

$$
0 \rightarrow k \rightarrow \hat{\mathscr{G}} \rightarrow \mathscr{G} \rightarrow 0
$$

such that for a certain lift $\lambda \rightarrow \hat{\lambda}$ of $\mathscr{G}$ to $\hat{\mathscr{G}}$, the commutation relations are

$$
\left[\hat{\lambda}_{1}, \hat{\lambda}_{2}\right]=\left[\widehat{\lambda_{1}, \lambda_{2}}\right]+\phi\left(\lambda_{1}, \lambda_{2}\right) \text {. }
$$

To be more precise, (164) is the condition under which (166) obeys the Jacobi identity.

There is no natural global choice of a cocycle obeying (164). But note that for $\lambda_{1}, \lambda_{2} \in \mathscr{G},\left\langle\lambda_{1}, d \lambda_{2}\right\rangle$ is a differential form. Upon picking some point $P \in X$, we can use the residue at $P$ of this differential form to define a cocycle:

$$
\phi_{P}\left(\lambda_{1}, \lambda_{2}\right)=\operatorname{Res}_{P}\left\langle\lambda_{1}, d \lambda_{2}\right\rangle \text {. }
$$


This makes sense on the completion $\mathscr{G}_{P}$ of $\mathscr{G}$ at $P$, and is easily seen (using the fact that the residues of an exact differential form are zero) to obey (164). Thus, for each $P$, we obtain a central extension

$$
0 \rightarrow k \rightarrow \hat{\mathscr{G}}_{P} \rightarrow \mathscr{G}_{P} \rightarrow 0 .
$$

The importance of the completion $\mathscr{G}_{\rightarrow} \mathscr{G}_{P}$ is that the $\mathscr{G}_{P}$ have a simple and general form. If $z$ is a local uniformizer at $P$ and $k((z))$ consists of formal series $\sum_{n=-N}^{\infty} a_{n} z^{n}, a_{n} \in k$, then $\mathscr{G}_{P} \simeq \mathscr{U} \otimes k((z))$. In this, the details of the particular algebraic curve are irrelevant locally at $P$. Thus, a basis for $\mathscr{G}_{P}$ consists of expressions $\alpha \otimes z^{n}$, with $n$ an integer and $\alpha \in \mathscr{U}$. In this basis, $\widehat{\mathscr{G}}_{P}$ may be described by saying that for $\alpha, \beta \in \mathscr{U}$ and $n, m \in Z$,

$$
\left[\alpha \otimes z^{n}, \beta \otimes z^{m}\right]=[\alpha, \beta] \otimes z^{n+m}+n c \delta_{n+m}\langle\alpha, \beta\rangle,
$$

where $\delta_{k}=1$ for $k=0$ and zero otherwise, and $c$ is a central element. Equation (169) is simply an affine Lie algebra over $k$.

So far, we have only constructed what one might regard as a local central extension of $\mathscr{G}$ at $P$. To work globally, we wish to combine these. Thus, we form the adelic products

$$
\widehat{\mathscr{G}}=\coprod_{P} \widehat{\mathscr{G}}_{P}
$$

An element of $\hat{\mathscr{G}}$ is a linear combination of objects $\oplus_{P} \lambda_{P}$, where $\lambda_{P}$ is in $\hat{\mathscr{G}}_{P}$ for all $P$, and $\lambda_{P}$ is regular at $P$ for all but finitely many $P$. Evidently $\hat{\mathscr{G}}$ is a central extension of the analogous adelic product $\coprod_{P} \mathscr{G}_{P}$. We identify the central elements $c$ in the various $\mathscr{G}_{P}$ so that the adelic extension is

$$
0 \rightarrow k \rightarrow \hat{\mathscr{G}} \rightarrow \coprod_{P} \mathscr{G}_{P} \rightarrow 0 .
$$

To study global properties of the algebraic curve $X$, it is important to consider the natural diagonal embedding

$$
\mathscr{G} \rightarrow \coprod_{P} \mathscr{G}_{P}
$$

which maps $\lambda \in \mathscr{G} \rightarrow \oplus_{P} \lambda_{P}$, with $\lambda_{P}=\lambda$ for all $P$. We map $\mathscr{G}$ into $\hat{\mathscr{G}}$ by embedding in $\coprod_{P} \mathscr{G}_{P}$ as in (172) and then lifting to $\hat{\mathscr{G}}$. For $\lambda \in \mathscr{G}$, let $\hat{\lambda}$ be the corresponding lift of $\lambda$ to $\hat{\mathscr{G}}$. The commutation relations of the $\hat{\lambda}$ are simply those of $\mathscr{G}$, since

$$
\begin{aligned}
{\left[\hat{\lambda}_{1}, \hat{\lambda}_{2}\right] } & =\left[\widehat{\lambda_{1}, \lambda_{2}}\right]+\sum_{P} \phi_{P}\left(\lambda_{1}, \lambda_{2}\right) \\
& =\left[\widehat{\lambda_{1}, \lambda_{2}}\right]+\sum_{P} \operatorname{Res}_{P}\left\langle\lambda_{1}, d \lambda_{2}\right\rangle, \\
& =\left[\widehat{\lambda_{1}, \lambda_{2}}\right],
\end{aligned}
$$

where we have again used the fact that the sum of the residues of a differential form is zero. Equation (173) shows that the central extension (171) splits (that is, 
becomes trivial) when restricted to the "global Lie algebra" $\mathscr{G}$, or more exactly when restricted to the image of $\mathscr{G}$ under the natural diagonal map to $\hat{\mathscr{G}}$. This is quite analogous to the behavior we found in discussing free fermions.

We now must consider representation theory of these Lie algebras. An irreducible representation $W_{P}$ of $\hat{\mathscr{G}}_{P}$ is said to be a highest weight representation if it contains a vector $v$ with

$$
\left(\alpha \otimes z^{n}\right) \cdot v=0, \text { for } n>0 \text { and } \alpha \in \mathscr{U} .
$$

We are interested in representations such that the space of vectors with that property is finite dimensional. $W_{P}$ is said to be unramified if there is a vector $1_{P}$ (necessarily unique up to multiplication by a scalar) such that

$$
\left(z^{n} \otimes \alpha\right) \cdot 1_{P}=0 \text { for } n \geqq 0 \text { and } \alpha \in \mathscr{U} \text {. }
$$

An automorphic representation of $\hat{\mathscr{G}}$ is a family of highest weight irreducible representations $\left\{W_{P} \mid P \in X\right\}$ such that $W_{P}$ is unramified for all but finitely many $P$. One then forms the adelic product

$$
W=\coprod_{P} W_{P}
$$

generated by sums $\oplus_{P} w_{P}$ with $w_{P} \in W_{P}$ for all $P$ and $w_{P}=1_{P}$ for all but finitely many $P$. In physical terminology, $W$ is the space of observables in a quantum field theory. Points $P$ such that $W_{P}$ is ramified are points at which there is an insertion of a "spin operator" 23 or a non-abelian generalization thereof.

$W$ in this situation automatically furnishes a representation of the adelic Lie algebra $\hat{\mathscr{G}}$. Thus for $\lambda_{P} \in \widehat{\mathscr{G}}_{P}$ and $w=\otimes_{P} w_{P}$, we simply let $\lambda_{P}$ act on the $P$ component of $w$ while leaving the others invariant; thus $\lambda_{P} \cdot w=\otimes_{Q} w_{Q}^{\prime}$ with $w_{Q}^{\prime}=w_{Q}$ for $Q \neq P$ and $w_{P}^{\prime}=\lambda_{P} \cdot w_{P}$. For $\lambda=\oplus_{P} \lambda_{P} \in \hat{\mathscr{G}}$, we define $\lambda \cdot w=\sum_{P} \lambda_{P} \cdot w$. This makes sense since $\lambda_{P} \cdot w=0$ for all but finitely many $P$.

Next, as in the case of free fermions, we wish to define the quantum field theory expectation values. This means that we wish to define a linear functional $\int: W \rightarrow k$ subject to certain conditions. For $\lambda \in \mathscr{G}$ (embedded in $\hat{\mathscr{G}}$ by the diagonal embedding) and $w \in W$, one requires

$$
\int \hat{\lambda} \cdot w=0 .
$$

This corresponds again to the Ward identities of "current algebra on a Riemann surface." As in our discussion of free fermions, the linear functionals $\int$ which obey (177) form a vector space which can be regarded as the dual space of the quotient $W / \mathscr{G} W$. It seems (though I will not attempt to prove it here) that the latter quotient always has a dimension that is positive but typically greater than one. ${ }^{24}$ Therefore,

\footnotetext{
${ }^{23}$ This concept originally appeared in the theory of the Ising model and lately has become important in string theory [15]

${ }^{24}$ An upper bound on the dimension of $W / \mathscr{G} W$ is $g \cdot \operatorname{dim} \mathscr{U}$, the product of the genus $g$ of $X$ with the dimension of the finite dimensional Lie algebra $\mathscr{U}$. This upper bound, which comes by using the Riemann-Roch theorem to count meromorphic functions with various poles, is exact if the representations $W_{P}$ are Verma modules, but in the more interesting case in which these are degenerate representations of the $\mathscr{G}_{P}$, the dimension of $W / \mathscr{G} W$ is smaller
} 
maps $\int: W \rightarrow k$ obeying (177) exist but are not uniquely determined, even up to multiplication by a scalar. In a particular physical context, one would usually have additional requirements beyond (177) that would enter in determining the map $\int$.

It is clear that the two examples of quantum field theory that we have discussed - namely free fermions, and current algebra - are closely analogous. Actually, the former can be used to provide an example of the latter, in the following way.

Let us first recall the spinor representation of the affine Lie algebra $\widehat{S O}(n)[16]$. Let $R$ be an $n$-dimensional vector space, with a basis $e_{i}, i=1 \ldots n$ and a nondegenerate quadratic form $\left\langle e_{i} \mid e_{j}\right\rangle=\delta_{i j}$. Form a Clifford algebra $\hat{R}$ by

$$
\left\{e_{i}, e_{j}\right\}=-2 \delta_{i j}
$$

The operators

$$
J_{i j}=\left[e_{i}, e_{j}\right] / 4
$$

obey the commutation relations of $S O(n)$. They generate, of course, the spinor representation of $S O(n)$. Let $\mathscr{U}$ be the Lie algebra spanned by the $J_{i j}$.

The analogous spinor representation of $\widehat{S O}(n)$ is constructed from an infinite dimensional Clifford algebra with basis $e_{i}^{v}, i=1 \ldots n$ and $v \in Z+\frac{1}{2}^{25}$. The anticommutation relations are

$$
\left\{e_{i}^{v}, e_{j}^{s}\right\}=-2 \delta_{i j} \delta^{v+s}
$$

We represent this Clifford algebra in a space $\mathscr{B}$ containing a highest weight vector $|\Omega\rangle$ with $e_{i}^{v}|\Omega\rangle=0$, for $v>0$; this representation is unique up to isomorphism.

Introducing a formal variable $z$, the affine Lie algebra $\widehat{\mathscr{G}} \simeq \widehat{S O}(n)$ is spanned as a vector space by $\mathscr{U} \otimes k\left[z, z^{-1}\right]$ plus a central element (with $\mathscr{U}$ the Lie algebra of $S O(n))$. To represent $S O(n)$ in the space $\mathscr{B}$, let $J_{i j} \otimes z^{n}$ be represented by

$$
J_{i j}^{n}=\frac{1}{4} \sum_{k}\left[e_{i}^{k}, e_{j}^{n-k}\right] .
$$

This can be seen [16] to obey the $\widehat{S O}(n)$ Lie algebra, with a central term.

To make contact with our discussion of free fermions, we must systematically repeat our earlier discussion with $L^{1 / 2}$ replaced by $L^{1 / 2} \otimes R, R$ an $n$-dimensional vector space with basis $e_{i}$ and quadratic form $\left\langle e_{i}, e_{j}\right\rangle=\delta_{i j}{ }^{26}$ Thus we replace $Y=\left\{\right.$ rational sections of $\left.L^{1 / 2}\right\}$ by $Z=\left\{\right.$ rational sections of $\left.L^{1 / 2} \otimes R\right\}$ and repeat the previous discussion. Completing $Z$ at $P \in X$, and calling the completion $Z_{P}$, we form the exterior algebra $\wedge Z_{P}$ as before. We then define a Clifford algebra $C Z_{P}$ as follows. For sections $f \otimes e_{i}$ and $g \otimes e_{j}$ of $L^{1 / 2} \otimes R$, we postulate the Clifford multiplication law

$$
\left\{f \otimes e_{i}, g \otimes e_{j}\right\}=-2 \delta_{i j} \operatorname{Res}_{P}(f g) .
$$

\footnotetext{
${ }^{25}$ This will give an unramified representation. Choosing $v \in Z$ gives a ramified representation. The $\frac{1}{2}$ here has the same origin it did in Eq. (3) of section I

${ }^{26}$ More generally, $R$ may be an $N$-dimensional vector bundle with a quadratic pairing $R \otimes R \rightarrow O_{X}$. Since we will be exhibiting a $G L(N, F)$ action $(F=$ function field of $X)$, and all vector bundles are equivalent over $F$, we content ourselves with the case of $R$ trivial
} 
Trivializing $L^{1 / 2}$ in a neighborhood of $P$ and choosing a uniformizer $z$ at $P$, a typical element of $Z_{P}$ is $\sum_{n=-N}^{\infty} a_{n} z^{n}$. Thus (182) is equivalent to the statement that

$$
\left\{z^{n} \otimes e_{i}, z^{m} \otimes e_{j}\right\}=-2 \delta_{i j} \operatorname{Res}_{P}\left(z^{n+m} d z\right)=-2 \delta_{i j} \delta^{m+n+1} .
$$

This coincides with (180) under $e_{i}^{v} \leftrightarrow z^{v-1 / 2} \otimes e_{i}$ (for $v \in z+\frac{1}{2}$ ). So the irreducible module $V_{P}$ for the Clifford algebra $C Z_{P}$ is also a highest weight module ${ }^{27}$ for the affine Lie algebra $\mathscr{G}_{P} \simeq \widehat{S O}(n)$, with

$$
J_{i j}^{n} \leftrightarrow \frac{1}{4} \sum_{r}\left[z^{r} \otimes e_{i}, z^{n-r-1} \otimes e_{j}\right] .
$$

The adelic space $V=\coprod_{P} V_{P}$, which we originally introduced as an irreducible module for the adelic Clifford algebra $C Z=\coprod_{P} C Z_{P}$ thus also furnishes an automorphic representation of the adelic Lie algebra $\widehat{\mathscr{G}}=\coprod_{P} \widehat{\mathscr{G}}_{P}$. We therefore have two notions of what we might require for the expectation value $\int: V \rightarrow k$. It may be compatible with the Clifford algebra in the sense of $(160)$ or with the Lie algebra in the sense of (177). These two notions are consistent, however (this is well known in a different language in the physics literature), essentially because the global Lie algebra $\mathscr{G} \subset \hat{\mathscr{G}}$ acts as an algebra of automorphisms of the global exterior algebra $(\wedge Z)^{+} \subset C Z$. The unique $\int: V \rightarrow k$ compatible with the Clifford algebra is also compatible with the Lie algebra.

The final subject that we will discuss here is one that is essential for making closer contact with the modern theory of automorphic forms. In addition to the Lie algebra of rational maps of $X$ into the Lie algebra of $S O(n)$, there is also a group $G$ of rational maps of $X$ into the group $S O(n, k)(G$ consists of orthogonal matrices whose matrix elements are rational functions). Clearly $G$ and $\mathscr{G}$ are closely related, but $\mathscr{G}$ is not the Lie algebra of $G$. (The exponential of a rational function in $\mathscr{G}$ would not be rational; $G$ has no Lie algebra and $\mathscr{G}$ has no Lie group.) One precise relation is that $G$ acts as a group of automorphisms of $\mathscr{G}$; for $g \in G$ and $\lambda \in \mathscr{G}, g$ maps $\lambda$ to $g^{-1} \lambda g$, preserving the $\mathscr{G}$ Lie algebra. It is natural to ask whether the $\mathscr{G}$ (and $\hat{\mathscr{G}}$ ) module $V$ admits a $G$ action compatible in this sense with the action of $\lambda$.

This question is mostly a local question, and as such the answer is known. For $P \in X$, let $G_{P}$ be the completion of $G$ at $P$. Rather like the Lie algebra $\mathscr{G}_{P}$, the group $G_{P}$ has a central extension by the multiplicative group $k^{*}$ of $k$ :

$$
0 \rightarrow k^{*} \rightarrow \hat{G}_{P} \rightarrow G_{P} \rightarrow 0 .
$$

What is more, the highest weight modules $V_{P}$ for the affine Lie algebra also admit a group action, with the Lie algebra and and group actions being compatible under conjugation $\lambda \rightarrow g^{-1} \lambda g[12,17]$. To be very explicit, this compatibility of the Lie algebra and the group means that if $\lambda \in \mathscr{G}_{P}$ and $g \in G_{P}$ are represented in $V_{P}$ by operators $R(\lambda)$ and $S(g)$, respectively, then

$$
R\left(g^{-1} \lambda g\right)=S(g)^{-1} R(\lambda) S(g) .
$$

${ }^{27}$ With two irreducible components 
In the case of the spinor representation of $\widehat{S O}(n)$ the group action is also compatible in the same sense with the action of the Clifford algebra $C Z_{P}$. This means that for $f \in \wedge Z_{P}$, with $\hat{f}$ denoting the lift to $C Z_{P}$, we have

$$
S(g)^{-1} \hat{f} S(g)=g^{-1} f g \text {. }
$$

Equations (186) and (187) are just the assertions that the $S(g)$ transform the Lie algebra $\mathscr{G}$ and the Clifford algebras $C Z_{P}$ as one would expect.

For each $P \in X$, let $K_{P}$ by the subgroup of $G_{P}$ consisting of rational maps of $X$ to $S O(n)$ (or whatever finite dimensional algebraic group we started with) that are regular at $P$. (If $k$ is a finite field, $K_{P}$ is the "maximal compact subgroup" of $G_{P}$.) In case $V_{P}$ is unramified, the vacuum vector $1_{P}$ is invariant under $K_{P} \subset G_{P}$.

These are the standard local facts. Passing now to the global situation, form the adelic product $\hat{G}=\coprod_{P} \hat{G}_{P}$. $\hat{G}$ consists of products $\otimes_{P} g_{P}$ with $g_{P} \in G_{P}$ for all $P$, and $g_{P} \in K_{P}$ for all but finitely many $P$. $\hat{G}$ acts irreducibly on $V=\coprod_{P} V_{P}$, the action of $\otimes_{P} g_{P} \in \hat{G}$ on $\otimes_{Q} v_{Q} \in V$ being $\otimes_{P} g_{P} \cdot \otimes_{Q} v_{Q}=\otimes_{P}\left(g_{P} \cdot v_{P}\right)$. (This definition is compatible with the "restricted product" definition of $V$, because $v_{P}=1_{P}$ for almost all $P$, $g_{P} \in K_{P}$ for almost all $P$. and $g_{P} \cdot 1_{P}=1_{P}$ if $g_{P} \in K_{P}$.)

Of course, $\hat{G}$ is a central extension of $\coprod_{P} G_{P}$ :

$$
0 \rightarrow k^{*} \rightarrow \hat{G} \rightarrow \coprod_{P} G_{P} \rightarrow 0 .
$$

We have also a diagonal embedding $\Delta: G \rightarrow \coprod_{P} G_{P}$. It is natural to ask whether (as in the Lie algebra case) this can be lifted to $\Delta^{\prime}: G \rightarrow \widehat{G}$ :

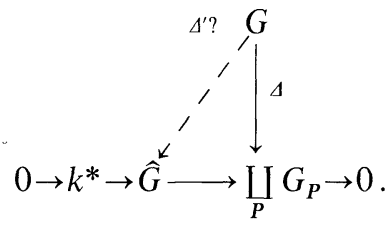

Of course, we can always define a map $\Delta^{\prime}$ making (189) commute. The issue is whether $\Delta^{\prime}$ can be chosen to be a group homomorphism or in other words whether the central extension (188) splits when restricted to $G \subset \coprod_{P} G_{P}$.

To show that it does split, pick first any lifting $\Delta^{\prime}$ of the diagonal embedding of $G$ in $\coprod_{P} G_{P}$. For $g \in G$, denote $\Delta^{\prime}(g)$ as $\hat{g}$. In general $\hat{g} \hat{h} \neq g h$; rather

$$
\hat{g} \hat{h}=a(g, h) \widehat{g h},
$$

where $a(g, h)$ is a cocycle. One wishes to know whether this cocycle splits in the sense that $a(g, h)=a(g) a(h) a^{-1}(g h)$ for some $a(g)$. If so, redefining $\hat{g} \rightarrow \tilde{g}=a^{-1}(g) \hat{g}$ will eliminate the cocycle from (190).

In the case of the spinor representation of $\widehat{S O}(n)$, we can easily prove the existence of such a splitting by using one dimensionality of $V /(\wedge Z)^{+} V$ and the compatibility of the group with the Clifford algebra action in the sense of Eq. (187). The latter implies that for $g \in G$, one has $\hat{g}^{-1}(\wedge Z)^{+} \hat{g}=(\wedge Z)^{+}$. As a result, the 
action of $\hat{g}$ on $V$ induces an action on the space $V /(\wedge Z)^{+} V$. Since that space is one dimensional, generated by the class $\overline{1}$ of the vacuum vector, it must be that for any $g \in G, \hat{g} \overline{1}$ is a multiple of $\overline{1}$, say

$$
\begin{gathered}
\hat{g} \overline{1}=a(g) \overline{1} \\
\widehat{h} \overline{1}=a(h) \overline{1} \\
\widehat{g h} \overline{1}=a(g h) \overline{1} .
\end{gathered}
$$

From the first two equations in (191), we see that $\hat{g} \widehat{h} \overline{1}=a(g) a(h) \overline{1}$. Comparing to the last equation in (191) and to (190) we see $a(g, h)=a(g) a(h) a^{-1}(g h)$. This is the desired result, showing that the central extension (188) splits when restricted to the diagonal.

This splitting of the central extension when restricted to the "global group," that is to $G \subset \hat{G}$, makes it possible to impose Ward identities for the group action. Ward identities for group action are much more obvious intuitively than Ward identities for exterior algebra or Lie algebra actions as discussed earlier. One simply requires that the correlation functions should be $G$-invariant; in other words that for $g \in G$ and $v \in V$, one requires

$$
\int \hat{g} \cdot v=\int v .
$$

This is possible only because of the splitting of the central extension; if we had $\hat{g} \cdot \hat{h}$ $=a(g, h) \cdot \widehat{g h}$ with $a(g, h) \neq 1$, it would be impossible for correlation functions to be simultaneously invariant under $g, h$, and $g h$.

I will conclude this section by briefly discussing the general context in which the discussion can be carried out. Let $W$ be an $O(N)$ bundle, that is a bundle endowed with a quadratic form $():, W \otimes W \rightarrow O_{X}\left(O_{X}\right.$ a trivial line bundle). Consider a fermion field $\Psi$ which is a section of $W \otimes L^{1 / 2}$. The Lagrangian is

$$
\mathscr{L}=\int_{X}\langle\Psi, \bar{D} \Psi\rangle
$$

Let $F$ be the field of rational functions on $X$, and let $U$ be the group $O(N, k)$, i.e., orthogonal $N \times N$ matrices with entries in $k$. If $W$ is trivial, let $G=O(N, F)$ be the group of $N \times N$ orthogonal matrices with entries in $F$; and more generally, let $G$ be the group of rational gauge transformations of the bundle $W$ which preserve the quadratic form $($, ). For $g \in G$, consider the transformation

$$
\Psi \rightarrow g \Psi
$$

Formally, this is a symmetry of (193), if we ignore the fact that $g$ might have poles or zeros at isolated points $P_{i} \in X$ and so is not in general a well defined transformation of the field variables. In Sect. (2) we analyzed this problem rather explicitly for the case $N=2$, and found that although not really a symmetry of the Lagrangian, (194) 
does lead to a well-defined transformation law of observables. ${ }^{28}$ The transformation by $g$ behaves as a "creation operator" at points $P_{i}$ where $g$ has poles. The resulting statements were the multiplicative Ward identities of Sect. 2.

The global splitting of (189) just described means that we have also for $N>2$ a rule for the transformation law of determinants and correlation functions under the group $G$. This transformation law is precisely a non-abelian generalization of the multiplicative Ward identities of Sect. 2 - though it would be hard to write them so explicitly as we did in the abelian case.

In fact, we learned in Sect. 2 that the multiplicative Ward identities lead naturally to an understanding of free fermions that are sections of $L^{1 / 2} \otimes E$ (with $E$ being some line bundle) in terms of free fermions that are sections of $L^{1 / 2}$. It is logical to ask whether likewise in the non-abelian case the multiplicative Ward identities, or in other words the $G$ action on the space $V=\coprod_{P} V_{P}$ of observables, lead to a relation between free fermion theories constructed using different bundles. It is easy to see that this is so. The key point is that every vector bundle $W$ is equivalent if one is only interested in rational functions and one does not care whether one's rational functions have zeros or poles. Very explicitly, let $\Psi^{i}$ be the components of $\Psi$, and let $g_{j}^{i}, j=1 \ldots N$ be $N$ rational sections of $W$. (In other words, for each fixed $j$, the $N$ component object $g_{j}^{i}, i=1 \ldots N$, is a rational section of $W$.) We then make the change of variables

$$
\Psi^{i}=g_{j}^{i} \widetilde{\Psi}^{j}
$$

The $\widetilde{\Psi^{j}}$ are then sections of an $N$ dimensional trivial bundle $\tilde{W}$, which will be endowed with some quadratic form which one finds by transforming the original quadratic form on $W$ by (195). Thus, once one knows a transformation law for correlation functions under a rational change of variables, it is immediate that determinants and correlation functions for any bundle can be computed in terms of those for the trivial bundle with a suitable quadratic form.

While the choice of bundle $W$ is irrelevant in this sense, this is far from true for the choice of quadratic form (, ). If we trivialize $W$ by a rational change of variable, $W$ is just $O_{X} \otimes R$, with $R$ an $N$ dimensional vector space over $k$; and $\Gamma(W)$, the space of rational sections of $W$, is just an $N$ dimensional vector space $\hat{R}$ over the field $F$ of rational functions. Likewise $\Gamma\left(O_{X}\right)$, the space of rational sections of $O_{X}$, is a one dimensional vector space $O$ over $F$. If $f$ and $g$ are rational sections of $W$, a quadratic form ( , ) on $W$ gives a map $f, g \rightarrow(f, g)$ of $\Gamma(W) \otimes \Gamma(W) \rightarrow \Gamma\left(O_{X}\right)$. This map is just a quadratic form $(,)_{F}$ on the $F$-vector space $\hat{R}$, i.e. a symmetric $F$-linear map $\hat{R} \otimes \hat{R} \rightarrow O$, or more explicitly a symmetric $N \times N$ matrix $M$ with entries in $F$. While all bundles are equivalent over $F$, it is not so for quadratic forms. The quadratic form (, ) has a discriminant $S=\operatorname{det} M$ and is nontrivial if $S$ is not a square in $F$. (Depending on $k,($,$) may have other invariants as well.)$

The essential generalization of the theory of free fermions as described above is thus that one can consider a nontrivial quadratic form (, ). If, say, $N=1$, a

\footnotetext{
${ }^{28}$ In Sect. (2), we studied $G=U(1)$, and considered zeros and poles of $g$ separately; for $G=O(2)$, the determinant of every $g \in G$ is 1 so the zeros and poles occur at the same points $P_{i}$ (in different matrix elements of $G$ ). The difference is that what in Sect. (2) were called $\psi$ and $\tilde{\psi}$ are here being combined in a column vector $\Psi$
} 
quadratic form is just a rational function $S$; the Lagrangian is just $\mathscr{L}=\int S^{-1} \psi \bar{D} \psi$ and the canonical commutation relations (145) (in the space $V_{P}$ of observables at $P$ ) are replaced by

$$
(f, g)_{P}=-2 \operatorname{Res}_{P}(S f g) .
$$

If $S$ is a square in the completion $F_{P}$ of $F$ at $P$, this can be absorbed in $f \rightarrow S^{-1 / 2} f$, $g \rightarrow S^{-1 / 2} g$. If, however, $S$ has a zero of odd order at $P$, say $S=z^{n}$ with $n$ odd and $z$ a local uniformizer at $P$, then the formula $(f, g)_{P}=-2 \operatorname{Res}_{P}\left(z^{n} f g\right)$ is for definitely not equivalent to (145). If say, $n=-1$, then (196) becomes

$$
\left\{z^{n}, z^{m}\right\}=-2 \delta^{m+n},
$$

and this shows that at $P$ one is working in what physically would be called the Ramond sector. (The Neveu-Schwarz sector is $\left\{z^{n}, z^{m}\right\}=-2 \delta^{m+n+1}$. Here $m$ and $n$ are integers.) In mathematical terminology, what happens for odd $n$ is that the local module $V_{P}$ is "ramified" in the language of the theory of automorphic forms. Again in physical terminology, recalling that $V_{P}$ is the space of observables at $P$, we observe that the ramification points are points with insertion of a "spin operator."

Though I will not try to do so here, it is a natural guess that if $X$ is a curve over a finite field $k$, then the quadratic reciprocity law for the quadratic extension field $F[y] /\left(y^{2}-f\right)$, with $f \in F$, should have a proof by studying free fermions with discriminant $S=f$. To prove higher order reciprocity laws (and conceivably nonabelian ones), one must probably study other quantum field theories on $X$.

\section{Back to Grassmannians}

In Sects. I and II, we developed certain aspects of the relation of quantum field theory with the infinite Grassmannians studied in [1]. In Sect. III, we formulated the simplest quantum field theories in an algebraic language, closely related in fact to the modern theory of automorphic forms. In this section, we will attempt to discuss the relation between these subjects.

Let $F$ be the field of global meromorphic functions on $\Sigma$. For $P \in \Sigma$, let $F_{P}$ consist of functions meromorphic in a neighborhood of $P,{ }^{29}$ and let $K_{P}$ be the subring of $F_{P}$ consisting of functions regular at $P$. Let $G L(N, F)$ be the group of invertible $N \times N$ matrices with entries in $F$. Likewise, let $G L\left(N, F_{P}\right)$ and $G L\left(N, K_{P}\right)$ be the group of invertible $N \times N$ matrices with values in $F_{P}$ or $K_{P}$. Thus $K_{P}, F \subset F_{P}$ and $G L\left(N, K_{P}\right), G L(N, F) \subset G L\left(N, F_{P}\right)$. Finally, define the adelic ring $A=\coprod_{P} F_{P}$ to consist of products $\coprod_{P} f_{P}$, with $f_{P} \in F_{P}$ for all $P$ and all but finitely many $f_{P} \in K_{P}$. There is a diagonal embedding $F \rightarrow A$ given by $f \rightarrow \coprod_{P} f_{P}$ with $f_{P}=f$ for all $P$; this is allowed since each $f \in F$ has only finitely many poles. Let $G L(N, A)$ consist of invertible $N \times N$ matrices with entries in $A$. The embedding of $F$ in $A$ gives an embedding of $G L(N, F)$ in $G L(N, A)$.

\footnotetext{
${ }^{29}$ To work over an arbitrary ground field $k$, one must take $F_{P}$ to consist of formal power series around $P$, i.e., the completion at $P$. In this section, for brevity, we will permit ourselves where convenient to assume that the ground field is the field of complex numbers
} 
We work on a Riemann surface $\Sigma$ (over $C$ ) with the $G L(N)$ invariant theory

$$
\mathscr{L}=\frac{i}{\pi} \int \tilde{\psi}_{i} \bar{D} \psi_{i}
$$

Here $\psi_{i}, i=1 \ldots N$ are $N$ sections of $L^{1 / 2}$, transforming under $G L(N)$ as $\psi \rightarrow g^{-1} \psi$ (the use of $g^{-1}$ rather than $g$ will standardize later formulas), while $\tilde{\psi}^{i}, i=1 \ldots N$ are $N$ sections of $L^{1 / 2}$, transforming under $G L(N)$ as $\tilde{\psi} \rightarrow g^{T} \tilde{\psi}$. [The theory (198) really has an $O(2 N)$ symmetry, but considering only the $G L(N)$ subgroup will be adequate for illustrative purposes and simpler. We could more generally regard $\psi$, $\tilde{\psi}$ as sections of $L^{1 / 2} \otimes W, L^{1 / 2} \otimes \tilde{W}$ with $W$ an arbitrary rank $N$ vector bundle and $\widetilde{W}$ its dual. Because of the $G L(N, F)$ action proved at the end of the last section and the fact that every vector bundle is trivial if one works with meromorphic rather than regular sections, this generalization is not too essential; the dependence on $W$ is really implicit in the $G L(N, F)$ action.]

Suppose that we delete a point $P$ from $\Sigma$. We then need boundary conditions on the $\psi_{i}$ (and dual boundary conditions, which we will not discuss explicitly, on the $\left.\tilde{\psi}_{j}\right)$ near $P$. The standard boundary conditions are to require at each $P$

$$
\int_{D}\left|\psi_{i}\right|^{2}<\infty
$$

with $D$ a small disc around $P$. The theory with these boundary conditions is equivalent to the theory with $P$ not deleted. More generally, we can take

$$
\int_{D}\left|g^{-1} \psi\right|^{2}<\infty
$$

for some $g \in G L\left(N, K_{P}\right)$. ( $D$ is chosen, depending on $g$, to contain no singularities of $g$ except perhaps at $P$.) The boundary condition (199) is equivalent to (200) if and only if $g$ is regular at $P$, i.e. if and only if $g \in G L\left(N, K_{P}\right)$. More generally, if $g, g^{\prime} \in G L\left(N, F_{P}\right)$, the use of $g$ or $g^{\prime}$ in (200) gives equivalent conditions on $\psi$ if and only if $g=g^{\prime} h$ with $h$ regular at $P$, i.e. $h \in G L\left(N, F_{P}\right)$. Thus, the space of boundary conditions that we can obtain in this way is isomorphic to the quotient space $G L\left(N, F_{P}\right) / G L\left(N, K_{P}\right)$.

This quotient space, which we will call $G r_{P}$, does not coincide with the Grassmannian as studied in Sect. I, but is closely related. In fact $G r_{P}$ corresponds to the subspace of the Grassmannian of Sect. I that Segal and Wilson call $\mathrm{Gr}^{(n)}$. Thus, we can immediately restrict the construction of the DET bundle reviewed in Sect. I to give a line bundle over $G r_{P}$ which we will call $\mathrm{DET}_{P}$. What is more, according to our discussion in Sect. 1, the space $V_{P}$ of holomorphic sections of the dual bundle DET* over $G r_{P}$ is isomorphic to the Hilbert space of the quantum field theory (198) or in other words to the space of observables that can be inserted at $P$. We recall that there is a particularly natural "vacuum" section of $G r_{P}$ which we will call $1_{P}$.

Now we wish to delete not just a single point $P$ but an arbitrary finite collection of points $P_{1} \ldots P_{n}$. A uniform way to describe the resulting freedom in choice of boundary conditions is to say that we pick for each $P \in \sum$ a point $\lambda_{P} \in G r_{P}$, with the proviso that for all but finitely many $P, \lambda_{P}$ is the standard point $H_{P(+)}$ in $G r_{P}$, corresponding to (199). Thus, an allowed boundary condition is a point in

$$
\overline{G r}=\coprod_{P} G r_{P}=\coprod_{P} G L\left(N, F_{P}\right) / G L\left(N, K_{P}\right) .
$$


The symbol $\prod_{P} G r_{P}$ simply refers to products $\prod_{P} \lambda_{P}$ with almost all $\lambda_{P}$ equal to $H_{P(+)}$. Over $\frac{P}{G r}$, we define the line bundle $\mathrm{DET}=\otimes_{P} \mathrm{DET}_{P} . \overline{G r}$ is a union of subspaces $G r_{P_{1} \ldots P_{n}}=\prod_{i=1}^{N} G r_{P_{i}} \cdot \prod_{Q \notin\left(P_{1} \ldots P_{n}\right)} H_{Q(+)}$, and a section of DET is considered holomorphic if it is holomorphic on each $G r_{P_{1} \ldots P_{n}}$.

The group $G L\left(N, F_{P}\right)$ does not act on $\mathrm{DET}_{P}$, but only a central extension of it; and likewise, it is a certain central extension of $G L(N, A)$ that acts on DET. However, if $G L(N, F)$ is embedded in $G L(N, A)$ by the diagonal embedding $\left(g \rightarrow \prod_{P} g_{P}\right.$ with $g_{P}=g$ for all $\left.P\right)$, then the central extension of $G L(N, A)$ becomes trivial when restricted to $G L(N, F)$, as we saw at the end of the last section. This means that upon dividing $G r$ by $G L(N, F)$, the DET line bundle descends to a line bundle on the quotient. Explicitly, the quotient of $G r$ by $G L(N, F)$ is the double coset space

$$
\mathscr{M}=G L(N, F) \backslash G L(N, A) / \prod_{P} G L\left(N, K_{P}\right) .
$$

An element of $\mathscr{M}$ is an element $\coprod_{P} g_{P} \in G L(N, A)$ with $\coprod_{P} g_{P}$ equivalent to $\coprod_{P}\left(h g_{P} k_{P}\right)$, for any $h \in G L(N, F)$ and $k_{P} \in G L\left(N, K_{P}\right)$. It is a standard fact that $\mathscr{M}$ is isomorphic to the moduli space of rank $N$ vector bundles over $\Sigma{ }^{30}$

A holomorphic section of DET* over $\mathscr{M}$ is a section of DET* on $\overline{G r}$ that is $G L(N, F)$ invariant. There is one such holomorphic section that arises in a completely natural and canonical way. Observing that a point in $\overline{G r}$ corresponds to a system of boundary conditions on the $\bar{D}$ operator, the fermion Pfaffian $\operatorname{Pf}(\bar{D})$ is a natural section of DET* over $\overline{G r}$. It is $G L(N, F)$ invariant, this being essentially the content of the multiplicative Ward identities of Sect. II.

More explicitly, let $\prod_{P} g_{P}$ define a point in $\overline{G r}$. The corresponding boundary conditions are that $g_{P}^{-1} \psi$ should be square integrable near $P$ for each $P$. For $h \in G L(N, F)$, the transformation $\psi \rightarrow h^{-1} \psi, \tilde{\psi} \rightarrow h^{T} \tilde{\psi}$ preserves the Lagrangian, once we delete a finite set of points at which $h$ or $h^{-1}$ is not regular, and maps the condition of square integrability of $g_{P}^{-1} \psi$ to square integrability of $\left(h g_{P}\right)^{-1} \psi$. This is the boundary condition associated with $\coprod_{P} h g_{P} \in \overline{G r}$. So the action of $h \in G L(N, F)$ on $\overline{G r}$ changes the Dirac boundary condition in a way that can be absorbed in $\psi \rightarrow h^{-1} \psi, \tilde{\psi} \rightarrow h^{T} \tilde{\psi}$. As a result, the Dirac determinant is invariant under the action of $G L(N, F)$.

\footnotetext{
${ }^{30}$ The vector bundle $W$ associated to $\coprod_{P} g_{P} \in G L(N, A)$ may be described by saying that an $N$-plet of meromorphic functions $\psi_{i}$ is to be considered a regular section of $W$ at $P$ if $g_{P}^{-1} \psi$ is regular at $P$ in the usual sense. The vector bundle associated in this way with $\coprod_{P} g_{P}$ is readily seen to be isomorphic to the one associated with $\coprod_{P} h g_{P} k_{P}$, the isomorphism being $\psi \rightarrow h \psi$. Thus a point $m \in \mathscr{M}$ gives rise to a unique isomorphism class of vector bundles over $\Sigma$. That every vector bundle $W$ over $\Sigma$ arises from some $m \in \mathscr{M}$ is proved by first finding $N$ linearly independent global meromorphic sections $\psi=\left(\psi_{1}, \ldots, \psi_{N}\right)$ of $W$. Then one picks at each $P$ a basis $u_{P}=\left(u_{1}^{P} \ldots u_{N}^{P}\right)$ of sections of $W$ regular and linearly independent at $P$, and defines $g_{P}$ by $u_{P}=g_{P} \psi$
} 
These observations have close counterparts in the theory of automorphic representations. (See [7] for an introduction.) The adelic space $V=\bigsqcup_{P} V_{P}$ of holomorphic sections of DET* over $\overline{G r}$ is an automorphic representation of the adele group $G L(N, A)$, and the Dirac determinant $\operatorname{Pf}(\bar{D})$ is a distinguished $G L(N, F)$ invariant vector in this space which plays the role of an automorphic form associated with a given automorphic representation. The double coset space $\mathscr{M}$ is analogous to double coset spaces that arise in the theory of automorphic representations. While these observations do not constitute the solution of any problem concerning either automorphic representations or conformal field theory, the observation of the existence of a relation between these fields, which we have tried to make in this section and the last one, comes as a surprise and may hopefully help stimulate further developments in future.

\section{Some Remarks on String Field Theory}

String field theory is usually formulated in terms of a string functional $A\left(X^{\mu}(\sigma)\right.$, $b(\sigma), c(\sigma))$ ( $b$ and $c$ are the ghosts). Although some structure playing the role sometimes postulated for string field theory is presumably necessary, string field theory as presently formulated involves ugly elements like delta function overlaps of strings which hopefully can be eliminated in the future. To eliminate them it is probably necessary to introduce new degrees of freedom. Such new degrees of freedom must of course enlarge the gauge invariance without changing the physical content.

One line of thought begins with the observation that the ghosts are really left invariant differential forms on the Virasoro group manifold, i.e. the manifold of $G=\operatorname{diff} S^{1}$. If $G$ were a compact Lie group, the de Rham cohomology of the $G$ manifold would coincide with the cohomology of the Lie algebra $\mathscr{G}$ (computed by studying the left invariant differential forms). Thus, this is a situation in which "new bosonic degrees of freedom," the motion on the group manifold, can be introduced without changing the "on shell physical theory," i.e. the cohomology. And the cohomology of the group manifold is surely a more "geometrical" notion than the formal Lie algebra cohomology of left invariant vector fields. Unfortunately, in the case of $G=\operatorname{diff} S^{1}$ it seems that the group and Lie algebra cohomology do not coincide. More generally, in this discussion we could consider a homogeneous space $\operatorname{diff} S^{1} / H, H$ a subgroup of diff $S^{1}$. Again, introducing the motion on $\operatorname{diff} S^{1} / H$ as a new degree of freedom in string field theory seems to change the physical content unacceptably.

Recently, Bowick and Rajeev [18] attempted to formulate string field theory on the manifold diff $S^{1} / S^{1}$. Their very interesting proposal is in a rather different direction from including the group manifold as a dynamical degree of freedom; they require left invariance under $\operatorname{diff} S^{1}$ and reduce everything to computation at the origin in $\operatorname{diff} S^{1} / S^{1}$.

A related line of thought begins with the observation that the space of string fields $A[X, b, c]$ is equivalent to the space of local operators that can be inserted at a point $P$ on a Riemann surface $\Sigma$. To actually pick an identification of $A[X, b, c]$ with observables at a point on a Riemann surface requires, however, not just a 
choice of $P$ but also the choice of a uniformizer or local parameter $z$ at $P$. The latter is needed because (except for conformal fields of spin zero) a local operator $O(P)$ at $P$ is not invariant under reparametrizations that might be carried out at $P$. Instead of regarding the string field as a mere functional $A[X, b, c]$, one might try to let it "come to life" as an actual operator on a surface $\Sigma$. One might suppose that in the "classical string field Lagrangian" $\Sigma$ should have genus zero, while higher genus counterparts of whatever geometrical structures are used in the classical theory are likely to enter in the quantum perturbation theory.

This idea is closely related to the suggestion made above about including the motion on the group manifold as a dynamical degree of freedom, since according to the appendix of [19], the moduli space $\mathscr{N}$ of a Riemann surface $\Sigma$ of genus $g$ with a point $P$ and a local parameter $z$ at $P$ admits a formally transitive action of the Virasoro algebra (i.e., the tangent space at a generic point $x \in \mathcal{N}$ is spanned by the vector fields that generate the Virasoro algebra). This means that $\mathscr{N}$ is formally $G / H$, with $G$ the Virasoro group and $H$ the subgroup of $G$ that leaves fixed $x \in \mathscr{N}$. Thus, trying to let the string field "live" at a point $P$ on $\Sigma$ with arbitrary choice of $z$ is very similar to including the group manifold, or at least a homogeneous space thereof, as a dynamical degree of freedom.

Once one tries to think of the string field as an observable $O(P)$, it is natural to go a little bit farther and do what one actually does both in quantum field theory and in the modern theory of automorphic forms, namely introduce an arbitrary product

$$
\prod_{i} O_{i}\left(P_{i}\right)
$$

of local observables $O_{i}$ at points $P_{i}$ on $\Sigma$. Of course, at this stage, we are including infinitely many copies of the basic physical space which is already adequately represented by a single local operator $O(P)$. Therefore, we will need gauge invariances that create and annihilate the points $P_{i}$ at which there are operator insertions, to tame the redundancy in describing the space of physical observables in string theory by a product such as (203). The gauge invariances that would create and annihilate the $P_{i}$ would presumably be along the lines of the multiplicative Ward identities of Sects. 2 and 4, though I do not have a specific proposal to make here.

In one approach to string field theory [20], one describes that subject in terms of a generalized cohomology ring, the basic ingredients being a derivation $Q$, an integration $\int$, and a product $*$. Let us see how far we can get along these lines if the string field is to be an arbitrary adelic product of local observables as in (203).

For $Q$ we have no problem. Given the basic linear transformation $O(P)$ $\rightarrow Q O(P)$ of individual local operators, we extend to arbitrary finite products by requiring $Q$ to be a derivation,

$$
Q\left(\prod_{i} O_{i}\left(P_{i}\right)\right)=\sum_{j}\left(O_{1}\left(P_{1}\right) \ldots\left(Q O_{j}\left(P_{j}\right)\right) \ldots O_{n}\left(P_{n}\right)\right) .
$$

Likewise, for integration there is a completely natural candidate, namely the Feynman path integral,

$$
\int O_{1}\left(P_{1}\right) \ldots O_{n}\left(P_{n}\right)=\int \mathscr{D} X \mathscr{D} b \mathscr{D} c e^{-I} \prod O_{i}\left(P_{i}\right) .
$$


This integration is such that "the integral of a total derivative is zero," i.e. $\int Q\left(\prod O_{i}\left(P_{i}\right)\right)=0$.

What about multiplication? Given $A=\prod O_{i}\left(P_{i}\right)$ and $B=\prod O_{j}\left(Q_{j}\right)$, as long as $P_{i} \neq Q_{j}$ for all $i, j$ there is a perfectly natural candidate for $A * B$, namely the product

$$
\prod O_{i}\left(P_{i}\right) \cdot \prod O_{j}\left(Q_{j}\right)
$$

in the naive sense. Clearly $Q$ as defined above is a derivation of this multiplication law. Expression (206) breaks down, however, if $P_{i}=Q_{j}$ for some $i, j$, because there is no natural way to multiply quantum field operators at coincident points. As a result, I cannot propose a general definition of $*$. Nevertheless, it is plausible that there may eventually be some way to overcome these problems and formulate string field theory "adelically."

\section{Appendix}

Let $\Sigma$ be a Riemann surface and $\mathscr{S}$ the Lie algebra of meromorphic fields on $\Sigma$. In [19], a certain central extension $\hat{\mathscr{S}}$ of $\mathscr{S}$ was described. The construction made use of formal pseudo-differential operators. Our purpose here is to give an alternative explicit description of the central extension $\mathscr{S}$. Apart from making contact with the results of [19], this will enable us to illustrate in a concrete and interesting context the properties of operator-valued differential forms described in Sect. (3), and to repeat for the Virasoro algebra the purely algebraic description of conformal field theory which was given in Sect. (4).

The starting point is the Lie algebra diff $S^{1}$ of diffeomorphisms of the circle. A generator of this Lie algebra is a vector field $f(\theta)(d / d \theta)$, with $\theta$ an angular parameter, $0 \leqq \theta \leqq 2 \pi$. The Lie bracket is of course

$$
[f, g]=\left(f \frac{d g}{d \theta}-\frac{d f}{d \theta} g\right) .
$$

The universal central extension of this Lie algebra is described by a cocycle

$$
\phi(f, g)=\frac{1}{48 \pi} \int d \theta\left(f \frac{d^{3} g}{d \theta^{3}}-\frac{d^{3} f}{d \theta^{3}} g\right) .
$$

The normalization is conventional. Concretely, then, an element of the central extension $\overline{\operatorname{diff} S} S^{1}$ is a pair $(a, f)$, with $f$ a vector field on $S^{1}$ and $a$ a real number, and the Lie bracket being

$$
[(a, f),(b, g)]=\left(c \cdot \phi(f, g), f \frac{d g}{d \theta}-\frac{d f}{d \theta} g\right) .
$$

Here $c$ is an arbitrary real number, known in the physics literature as the "central charge." Equation (A3) obeys the Jacobi identity because $\phi$ obeys the cocycle condition

$$
\phi(f,[g, h])+\phi(g,[h, f])+\phi(h,[f, g])=0,
$$

for any three vector fields $f, g, h$ on $S^{1}$.

It is well known that the Lie algebra (A3) is closely related to a certain Lie algebra of meromorphic vector fields on the Riemann sphere $P^{1}$. The usual 
Virasoro algebra is the subalgebra of $\operatorname{diff} S^{1}$ consisting of pairs $(a, f)$ in which $f$ has a finite Fourier expansion

$$
f=\sum_{n=-N}^{+N} a_{n} e^{i n \theta}
$$

Such vector fields are finite linear combinations of

$$
L_{n}=i e^{i n \theta} \frac{d}{d \theta} \text {. }
$$

The commutators of the latter in $\widehat{\operatorname{diff}}^{1}$ are

$$
\left[L_{n}, L_{m}\right]=(n-m) L_{n+m}+\frac{c n^{3}}{12} \delta_{n+m}
$$

with $\delta_{k}=1$ for $k=0$ and zero otherwise. ${ }^{31}$ If one introduces the variable $z=e^{i \theta}$, then such vector fields have an analytic continuation throughout the complex $z$ plane, with possible poles at $z=0$ and $z=\infty$. Consequently, the Virasoro algebra may be interpreted as a central extension of the Lie algebra of meromorphic vector fields on the complex projective line $P^{1}$ with poles only at those two points.

We will here describe, for any compact Riemann surface $\Sigma$, a central extension $\hat{\mathscr{S}}$ of the Lie algebra $\mathscr{S}$ of meromorphic vector fields on $\Sigma$, such that (i) the definition of $\hat{\mathscr{S}}$ is local and intrinsic, not depending on arbitrary choices; (ii) if $\Sigma=P^{1}$, and we restrict ourselves to the subalgebra of $\mathscr{S}$ consisting of vector fields with poles only at $0, \infty$, then $\mathscr{S}$ reduces to the Virasoro algebra. The construction gives a different perspective on the results of [19].

The main problem is to determine the correct generalization of the cocycle (A2). The most naive idea is to try to interpret $\phi(f, g)$ as a residue of a differential form. Let

$$
u(z)=\frac{1}{24}\left(f \frac{d^{3} g}{d z^{3}}-\frac{d^{3} f}{d z^{3}} g\right)
$$

and let $U$ be the differential form

$$
U=u(z) d z .
$$

Then clearly

$$
\phi(f, g)=\operatorname{Res}_{z=0} U .
$$

Although the formulas above are correct, they cannot serve as an intrinsic local description of the Virasoro cocycle, because they depend on the local parameter $z$ at the point $z=0$ in $P^{1}$. The problem is that, although once we are given the vector fields $f, g$ and the local parameter $z$, no one can stop us from defining a differential form $U$ by (A 8) and (A9), the definition of $U$ definitely depends on the local parameter $z$. In fact, consider a charge of local parameter from $z$ to $w(z)$. A vector

\footnotetext{
${ }^{31}$ The Virasoro algebra is usually written with a cocycle $n^{3}-n$ in (A7) instead of $n^{3}$; the difference can be absorbed in a shift of $L_{0}$ (in other words, the two cocycles differ by a coboundary), and the formulas in this appendix will be shorter and more canonical if we work in the form (A7)
} 
field $f(z)(d / d z)$ transforms to $\hat{f}(\omega)(d / d w)$ with $\hat{f}=f \cdot(d w / d z)$. A little computation shows that $u(z) d z$ transforms to $\hat{u}(w) d w$, with

$$
\hat{u}(w)=\hat{f} \cdot \frac{d^{3} \hat{g}}{d w^{3}}-\frac{d^{3} \hat{f}}{d w^{3}} \cdot g+2\left(\hat{f} \frac{d \hat{g}}{d w}-\frac{d \hat{f}}{d w} \hat{g}\right)\{z, w\} .
$$

Here $\{z, w\}$ is the so-called Schwarzian derivative,

$$
\{z, w\}=\frac{d^{3} z / d w^{3}}{(d z / d w)}-\frac{3}{2} \cdot \frac{\left(d^{2} z / d w^{2}\right)^{2}}{(d z / d w)^{2}} .
$$

The first two terms on the right-hand side of (A11) are precisely what would be needed for $U$ to be a well-defined differential form, but the $\{z, w\}$ term ruins this.

If the $\{z, w\}$ term were not present in (A11), then the desired global generalization $\mathscr{S}$ of the Virasoro algebra would be obtained as follows. An element of $\hat{\mathscr{S}}$ would be a pair $(\lambda, f)$, with $f$ a vector field and $\lambda$ a differential form, and the composition law

$$
[(\lambda, f),(\eta, g)]=\left(\frac{c}{24}\left(f \frac{d^{3} g}{d z^{3}}-\frac{d^{3} f}{d z^{3}} g\right), f \frac{d g}{d z}-\frac{d f}{d z} g\right) .
$$

Of course, we have used a local coordinate $z$ in writing this. In the second term on the right-hand side of (A13), the expression $f g^{\prime}-f^{\prime} g$ is intrinsically defined object, independent of the choice of local coordinate $z$ - it is simply the commutator of vector fields $f, g$. But the $f g^{\prime \prime \prime}-f^{\prime \prime \prime} g$ term on the right-hand side of (A13) depends essentially on the choice of coordinate $z$ - as we have seen in (A12).

It turns out that (A13) should be reinterpreted (in a slightly different framework in which it is intrinsic and geometrical) rather than discarded. Let us recall the composition law for the Schwarzian derivative. If $u, w$, and $z$ are three local coordinates, the respective Schwarzian derivatives are related by

$$
\{u, z\}=\{u, w\}(d w / d z)^{2}+\{w, z\} .
$$

This means that if we define

$$
U_{w, z}=\left(\begin{array}{cc}
(d w / d z)^{-1} & \frac{c}{12}\{w, z\}(d w / d z)^{-1} \\
0 & (d w / d z)
\end{array}\right),
$$

then

$$
U_{u, w} U_{w, z}=U_{u, z}
$$

wherever $u, w$, and $z$ are all defined. Equation (A16) is a cocycle condition, which means that the $U$ 's can be interpreted as transition functions of a vector bundle. To be more exact, if we choose an open cover $\Sigma=\bigcup_{i} \Sigma_{i}$, with a local coordinate $z_{i}$ on $\Sigma_{i}$, then (A16) permits us to interpret the $U_{z_{i}, z_{j}}$ as transition functions on the intersection regions $\Sigma_{i} \cap \Sigma_{j}$. We will call the vector bundle so defined $\mathscr{V}_{0}$; it is a two dimensional sub-bundle of the infinite dimensional vector bundle called $\mathscr{V}$ in Sect. (3). 
We will denote a section of $\mathscr{V}_{0}$ as

$$
\left(\begin{array}{l}
\lambda \\
f
\end{array}\right) \text { or }(\lambda, f) \text {. }
$$

Locally, upon choosing a coordinate $z$, the objects $\lambda$ and $f$ are just a pair of complex functions. What is the right global description? In view of the form of the transition functions, what in the $z$ coordinate system is $(\lambda, f)$ is in the $w$ coordinate system

$$
\left((d w / d z)^{-1} \lambda+\frac{c}{12}(d w / d z)^{-1}\{w, z\} f,(d w / d z) f\right) .
$$

If one sets $c=0$, then (A18) is simply the transformation law for a differential form $\lambda$ and a vector field $f$; thus, at $c=0$, the pair $(\lambda, f)$ is a section of the bundle $L \oplus T$, with $L$ the canonical line bundle of $\Sigma$, and $T=L^{-1}$ the tangent bundle. At $c \neq 0$, the pair $(\lambda, f)$ are a section of a deformation $\mathscr{V}_{0}$ of $L \oplus T$.

The transition matrices (A15) are triangular, and this means that $\mathscr{V}_{0}$ is a vector bundle of a very special kind; it is an "extension of $T$ by $L$." In other words, there is an exact sequence

$$
0 \rightarrow L \rightarrow \mathscr{V}_{0} \rightarrow T \rightarrow 0 .
$$

This is simply the assertion that there is a natural map $\alpha$ from sections of $L$ to sections of $\mathscr{V}_{0}$, namely $\lambda \rightarrow(\lambda, 0)$; and a natural map $\beta$ from sections of $\mathscr{V}_{0}$ to sections of $T$, namely $(\lambda, f) \rightarrow f$. (Moreover, $\beta \circ \alpha=0$ and the image of $\alpha$ is the kernel of $\beta$.) For instance, the existence of $\beta$ is the statement that even at $c \neq 0, f$ in (A18) transforms as a vector field, although $\lambda$ does not transform as a differential form. There is, however, no natural map $f \rightarrow(?, f)$ from sections of $T$ to sections of $\mathscr{V}_{0}$, because there is no natural way to decide what? should be (if ? is zero in one coordinate system, it will not be zero in another). There is likewise no natural map $(\lambda, f) \rightarrow$ ? from sections of $\mathscr{V}_{0}$ to sections of $L$.

Let us pause for a slight digression. Actually, the extension (A19) is trivial. This is closely related to the uniformization theorem for Riemann surfaces. A uniformization of a Riemann surface $\Sigma$ gives a covering by open sets $\Sigma_{i}$ with local parameters $z_{i}$ such that on $\Sigma_{i} \cap \Sigma_{j}$ the transformations from $z_{i}$ to $z_{j}$ are $S L(2)$ transformations $z_{j}=\left(a z_{i}+b\right) /\left(c z_{i}+d\right)$. For such transformations the Schwarzian derivatives $\left\{z_{i}, z_{j}\right\}$ vanish, so the transition matrices (A18) become diagonal, and the extension $\mathscr{V}_{0}$ is split as $L \oplus T$. $^{32}$ Although the extension $\mathscr{V}_{0}$ can be trivialized or split, there is no intrinsic or natural way to do this. There are many ways to express this. On the one hand, although Riemann surfaces can be uniformized, there is no local way to find a uniformization; on the other hand, $\mathscr{V}_{0}$ actually has many splittings, which differ by global holomorphic sections of $\operatorname{Hom}(T, L)$ or in other words by holomorphic quadratic differentials. That the triviality of $\mathscr{V}_{0}$ is a more or less non-trivial global fact is also illustrated by the comment in the footnote. Since $\mathscr{V}_{0}$ cannot be split by any intrinsic local construction, the fact that it can be split

${ }^{32}$ It is also possible to turn this around and use the extension $\mathscr{V}_{0}$ as a tool in proving the uniformization theorem. In this approach, one first shows on cohomological grounds that $\mathscr{V}_{0}$ must split in genus $\geqq 2$, and then one uses a splitting of $\mathscr{V}_{0}$ in constructing a uniformization of $\Sigma$ 
globally is irrelevant in our aim of giving an intrinsic local description of a central extension of $\mathscr{S}$.

The introduction of $\mathscr{V}_{0}$ makes it possible now to give a satisfactory interpretation to the previous formula (A13), which we repeat for convenience:

$$
[(\lambda, f),(\eta, g)]=\left(\frac{c}{24}\left(f \frac{d^{3} g}{d z^{3}}-\frac{d^{3} f}{d z^{3}} g\right), f \frac{d g}{d z}-\frac{d f}{d z} g\right) .
$$

We saw in (A11) that if $(\lambda, f)$ and $(\eta, g)$ are regarded as sections of $L \oplus T$, then (A20) is not an intrinsic formula, but depends on the coordinate $z$. If, however, we interpret $F=(\lambda, f)$ and $G=(\eta, g)$ as sections of $\mathscr{V}_{0}$, then (A20) is coordinate independent. In other words, if one computes $[F, G]$ in the $z$ coordinate system by (A20) and then transforms to the $w$ coordinates by (A18), one gets the same result as if one transforms $F$ and $G$ to the $w$ coordinates by (A 18) before using (A 20) to compute $[F, G]$. To verify this is a relatively short calculation which we leave to the reader. $^{33}$

Thus, we have managed to find a locally defined bracket operation $[$,$] on$ sections of $\mathscr{V}_{0}$, but does it obey the Jacobi identity? Let $F=(\lambda, f), G=(\eta, g)$, and $H=(\varrho, h)$ be three sections of $\mathscr{V}_{0}$. One readily computes that

$$
[F,[G, H]]+[G,[H, F]]+[H,[F, G]]=(d U \cdot c / 12,0),
$$

where

$$
U(f, g, h)=\operatorname{det}\left(\begin{array}{lll}
f & g & h \\
f^{\prime} & g^{\prime} & h^{\prime} \\
f^{\prime \prime} & g^{\prime \prime} & h^{\prime \prime}
\end{array}\right)=\left(f \cdot \frac{d g}{d z} \cdot \frac{d^{2} h}{d z^{2}} \pm \text { permutations }\right) \text {. }
$$

It is easy to see that for meromorphic vector fields $f, g, h, U$ is a well-defined meromorphic function on $\Sigma$, independent of the choice of coordinate $z$. In (A21), $d U$ denotes the ordinary exterior derivative of the function $U$; this is a well-defined differential form, and as we have discussed earlier there is a well defined map $\phi \rightarrow(\phi, 0)$ of differential forms $\phi$ to sections $(\phi, 0)$ of $\mathscr{V}_{0}$.

Clearly, (A21) shows that the [,] operation is not a Lie algebra structure on sections of $\mathscr{V}_{0}$. However, the error is the "exact form" $(d U, 0)$. If we introduce an equivalence relation $\sim$ on sections of $\mathscr{V}_{0}$ by writing $F \sim G$ if $F-G=(d u, 0)$ for some meromorphic function $u$, and denote the space of equivalence classes as $\hat{\mathscr{S}}$, then (A21) is the Jacobi identify for $\hat{\mathscr{S}}$. Indeed, if we write the equivalence class of a section $F$ of $\mathscr{V}_{0}$ as $\bar{F}$, then clearly (A21) gives

$$
[\bar{F},[\bar{G}, \bar{H}]]+[\bar{G},[\bar{H}, \bar{F}]]+[\bar{H},[\bar{F}, \bar{G}]]=0 .
$$

$\hat{\mathscr{S}}$ with the Lie bracket $[$, ] is, finally, the Lie algebra that we have been aiming for. Its center consists of expressions $(\alpha, 0)$ with $\alpha$ a differential form, and $\alpha \sim \beta$ if $\alpha-\beta=d u$. Thus, let $\Omega^{1}$ denote the space of meromorphic one forms on $\Sigma$, and $\Omega^{0}$ the space of meromorphic functions; and let $d \Omega^{0}$ be the space of exact one forms, that is, one forms $\omega$ that can be written $\omega=d u$, with $u \in \Omega^{0}$. Then the center of $\hat{\mathscr{S}}$ can be identified as $\Omega^{1} / d \Omega^{0}$.

\footnotetext{
${ }^{33}$ While the operation $F, G \rightarrow[F, G]$ is thus coordinate independent, we have made heavy use of coordinates in describing this coordinate independent operation. Recently, a more obviously coordinate independent description has been given by P. Deligne (private communication)
} 
What about the quotient of $\hat{\mathscr{S}}$ by its center? Since a central element of $\hat{\mathscr{S}}$ is an element of the form $(\alpha, 0)$, taking the quotient by the center means identifying $(\alpha, f) \sim(\beta, f)$ for any $\alpha, \beta$, and $f$. The equivalence class of $(\alpha, f)$ in $\hat{\mathscr{S}} /$ (center) is thus uniquely determined by $f$, and the quotient of $\hat{\mathscr{S}}$ by its center can be identified as the Lie algebra $\mathscr{S}$ of meromorphic vector fields on $\Sigma$. Thus, we have constructed a central extension of Lie algebras of the form

$$
0 \rightarrow \Omega^{1} / d \Omega^{0} \rightarrow \hat{\mathscr{S}} \rightarrow \mathscr{S} \rightarrow 0 .
$$

This is the extension of Lie algebras described in [19], but quantum field theory is still lurking in the background. The real goal of the present appendix is to exhibit (A24) as a manifestation of the more general framework described at the end of Sect. 3. To this end, we consider instead of the sections $F=(\lambda, f), G=(\eta, g)$ of $\mathscr{V}_{0}$ the operator valued differential forms

$$
\widetilde{F}=\lambda \cdot 1+f \cdot T, \quad \widetilde{G}=\eta \cdot 1+f \cdot T .
$$

Here " 1 " is the identity operator and $T$ is the energy momentum tensor. To compute $\widetilde{F} \circ \widetilde{G}$ according to the recipe of Sect. (3), we must compute

$$
\tilde{F}(z) \cdot \widetilde{G}(w)=\sum_{n \geqq-N} O_{n}(w) \cdot(z-w)^{n} .
$$

Then $\tilde{F} \circ \tilde{G}(w)=O_{-1}(w)$. To compute $O_{-1}$, note that there are no short distance singularities in operator products $1 \cdot 1$ or $1 \cdot T$, while the singular part of the operator product $T(z) \cdot T(w)$ is well known to be

$$
T(z) \cdot T(w) \sim \frac{c}{2} \frac{1}{(z-w)^{4}}+\frac{2 T(w)}{(z-w)^{2}}+\frac{d T(w) / d w}{(z-w)}+\ldots .
$$

With the aid of this, we can evaluate

$$
\tilde{F} \circ \tilde{G}(w)=\frac{c}{12}\left(\frac{d^{3} f}{d w^{3}}\right) g(w) \cdot 1+2\left(\frac{d f}{d w}\right) g \cdot T(w)+f g \cdot d T(w) .
$$

In particular, the appearance of $d T(w)$ on the right-hand side of (A28) shows that the sections of $\mathscr{V}_{0}$ do not close under the operation. We could generalize $\mathscr{V}_{0}$ to include $d T$ as well as 1 and $T$; this would require a $3 \times 3$ generalization of (A 15), using higher order analogues of the Schwarzian derivative. One would still not get a closed system under the $\circ$ operation, since the $\circ$ operation applied to operator valued differential forms containing $d T$ would generate still higher operators.

However, we can get a closed operation on sections of $\mathscr{V}_{0}$ by defining ${ }^{34}$

$$
[\tilde{F}, \widetilde{G}]=-(\tilde{F} \circ \widetilde{G}-\tilde{G} \circ \widetilde{F}) / 2=\frac{c}{24}\left(\frac{d^{3} f}{d w^{3}} g-f \frac{d^{3} g}{d w^{3}}\right) \cdot 1+\left(f \frac{d g}{d w}-\frac{d f}{d w} g\right) \cdot T(w) .
$$

Clearly, under $(\lambda, f) \leftrightarrow \lambda \cdot 1+f \cdot T$, (A29) corresponds exactly with (A20), which we have thus placed in its quantum field theoretic context. That the $[$,$] operation$ defined in (A29) is well-defined is thus a consequence of the general properties of

\footnotetext{
${ }^{34}$ The minus sign in this definition is not essential in getting a Lie algebra, but makes the resulting
} formulas more standard 
the o operation discussed in Sect. (3). We also know from Sect. (3) that to get a Lie algebra it is necessary to take the quotient of the space $\Gamma(\mathscr{V})$ by the space of exact operator valued differential forms. Since $d(u \cdot 1+\beta \cdot T)=(d u \cdot 1+d \beta \cdot T+\beta \cdot d T)$, an operator valued differential form of the type $(\lambda \cdot 1+f \cdot T)$, with no $d T$ component, can be exact only if $f=0$ and $\lambda$ is exact in the usual sense. Therefore, we can identify from the rules of Sect. 3 the equivalence relation on the space of operator valued differential forms $\widetilde{F}=\lambda \cdot 1+f \cdot T$ that will lead to a Lie algebra structure. It is exactly the relation $\tilde{F} \sim \widetilde{G}$ if $\tilde{F}-\widetilde{G}=d u \cdot 1$, with $u$ an ordinary meromorphic function. The space of sections of $\mathscr{V}_{0}$ subject to this equivalence relation is precisely the space $\hat{\mathscr{S}}$ introduced above. The general analysis at the end of Sect. 3 correctly identifies $\Omega^{1} / d \Omega^{0}$ as the center of $\hat{\mathscr{S}}$. Thus, we have succeeded in exhibiting the extension (A24) as a consequence of a more general framework.

Clearly, the restriction to a finite dimensional subbundle $\mathscr{V}_{0}$ of $\mathscr{V}$ is not very representative of quantum field theory. Consideration of $\mathscr{V}$ instead of $\mathscr{V}_{0}$ would lead to Lie algebras much "larger" than (A24), but it is hard to be very explicit because regrettably one does not have a useable, concrete description of the complete operator algebra, i.e., the o operation on arbitrary sections of $\mathscr{V}$, even for free field theories. I will however briefly illustrate one simple example of a generalization of (A24). Consider the two component free fermion system with Lagrangian

$$
L=\frac{1}{\pi} \int \tilde{\psi} \bar{D} \psi .
$$

It is easy to see that identity operator together with the operators

$$
T_{n, m}=d^{n} \tilde{\psi} \cdot d^{m} \psi, \quad n, m=0,1,2, \ldots
$$

are closed under the operation. Let $\mathscr{V}_{1}$ be the subbundle of $\mathscr{V}$ corresponding to the operators 1 and $T_{n, m}$. So a section $A$ of $\mathscr{V}_{1} \otimes L$ is a meromorphic differential form which in a local coordinate system can be written

$$
A=\alpha \cdot 1+\sum_{n, m=0}^{N} a_{n, m} T_{n, m} .
$$

We will not try to describe here the generalization of the Schwarzian derivative that enters in the transformation of $A$ under change of coordinates.

Before trying to identify a Lie algebra, let us ask which are the exact forms. It is not too hard to see that any $A$ of the form (A32) can be written

$$
A=\alpha \cdot 1+\sum_{m=0}^{N^{\prime}} a_{m}^{\prime} T_{0, m}+d u,
$$

with $u$ some operator valued scalar. This means that taking the quotient by exact forms permits one to eliminate the $T_{n, m}$ for $n>0$. To see this, note that

$$
a_{n, m} d^{n} \tilde{\psi} d^{m} \psi=-\left(d a_{n, m}\right) d^{n-1} \tilde{\psi} \cdot d^{m} \psi-a_{n, m} d^{n-1} \tilde{\psi} \cdot d^{m+1} \psi+d\left(a_{n, m} d^{n-1} \tilde{\psi} \cdot d^{m} \psi\right)
$$

Repeated application of (A 34) permits one to eliminate all derivatives from $\tilde{\psi}$ and write $A$ in the form (A33).

Next, what is the operator algebra of the operators $T_{0, m}$ ? The answer is very striking and is perhaps most easily obtained in a canonical formalism. Working on 
a circle with angular parameter $\theta$, and using $\left\{\tilde{\psi}(\theta), \psi\left(\theta^{\prime}\right)\right\}=\pi \delta\left(\theta-\theta^{\prime}\right)$, one immediately sees

$$
\frac{1}{\pi}\left[\int d \theta f(\theta) T_{0, m}(\theta), \psi\left(\theta^{\prime}\right)\right]=f\left(\theta^{\prime}\right) \frac{d^{m} \psi}{d \theta^{m}} .
$$

This means that $\int d \theta f(\theta) T_{0, m} / \pi$ generates the transformation of $\psi(\theta)$ that we would usually associate with the differential operator

$$
f(\theta) \frac{d^{m}}{d \theta^{m}} .
$$

We can thus expect that under the o operation, the operator valued differential forms

$$
A=\alpha \cdot 1+\sum_{m} a_{m}^{\prime} \tilde{\psi} \frac{d^{m} \psi}{d z^{m}}
$$

obey the algebra of the differential operators

$$
\sum a_{m}^{\prime} \frac{d^{m}}{d z^{m}}
$$

More exactly, the correspondence between (A37) and (A38) only holds up to a central extension due to the identity operator " 1 ." We therefore reach the following expectation:

Let $\Sigma$ be a Riemann surface, and DIFF the Lie algebra of meromorphic differential operators on $\Sigma$. Then there is a central extension $\widehat{\mathrm{DIFF}}$ of DIFF, with center $\Omega^{1} / d \Omega^{0}$.

$$
0 \rightarrow \Omega^{1} / d \Omega^{0} \rightarrow \widehat{\mathrm{DIFF}} \rightarrow \mathrm{DIFF} \rightarrow 0 .
$$

The central extension $\hat{\mathscr{S}}$ of $\mathscr{S}$ constructed in (A24) is naturally a Lie sub-algebra of DIFF.

Clearly, much should be done to elucidate this more fully.

Some Applications. We conclude by briefly considering the Virasoro analogues of some constructions in Sects. 4 and 1. Thinking first of Sect. 4, let us formulate the Ward identities for the energy-momentum tensor in a way which

(i) makes sense on a Riemann surface of arbitrary genus;

(ii) makes sense over an arbitrary ground field $k$.

To this end, we simply imitate some of the definitions of Sect. 4. Thus, for every $P \in \Sigma$, let $\hat{\mathscr{S}}_{P}$ be the completion of $\hat{\mathscr{S}}$ at $P$. The $\hat{\mathscr{S}}_{P}$ are all isomorphic to a standard object, namely a certain completion of the Virasoro algebra in which one considers sums of $L_{n}$ 's of the form

$$
\sum_{n=-N}^{\infty} a_{n} L_{n}
$$

with $n$ bounded below but perhaps not above. A representation of $\hat{\mathscr{S}}_{P}$ is said to be of highest weight if it is of highest weight in the usual sense of Virasoro representation theory, and it is said to be unramified if it contains a unique vector 
$1_{P}$ annihilated by the $L_{n}, n \geqq-1$. An automorphic representation of $\hat{\mathscr{S}}_{P}$ is a family $\left\{V_{P}\right\}$ of highest weight representations of the $\hat{\mathscr{S}}_{P}$, with a common central charge and all but finitely many of them unramified.

One then defines the restricted product

$$
V=\coprod_{P} V_{P}
$$

consisting of products $\otimes_{P} v_{P}$, with $v_{P} \in V_{P}$ for all $P$ and almost all $v_{P}=1_{P}$. Likewise, let us define the adelic Lie algebra

$$
\hat{\mathscr{S}}=\coprod_{P} \hat{\mathscr{S}}_{P}
$$

to consist of formal sums $\oplus_{P} S_{P}$, with $s_{P} \in \hat{\mathscr{S}}_{P}$ for all $P$ and $s_{P}$ regular at $P$ for all but finitely many $P$. Then the "space of observables" $V$ admits a natural $\tilde{\mathscr{S}}$ action; $\tilde{\mathscr{S}}$ simply acts on $V$ componentwise, just as in similar situations considered in Sect. 4.

The Lie algebra $\mathscr{S}$ cannot be embedded in any of the $\hat{\mathscr{S}}_{P}$, because of the central extension. In fact, upon picking a local parameter at $P$, one can map $f \in \mathscr{S}$ to

$$
\hat{f}_{P}=(0, f) \in \hat{\mathscr{S}}_{P},
$$

but the $\hat{f}_{P}$ do not obey the $\mathscr{S}$ Lie algebra (but a central extension thereof). In trying to work globally we have a problem that did not have an analogue in Sect. $4:$ the $\hat{f}_{P}$ are not canonically defined, but depend on a choice of a local parameter at $P$. One way to proceed is to uniformize the curve $\Sigma$; this will induce a family of local parameters $\left\{z_{P} \mid P \in \Sigma\right\}$ which are uniquely determined up to $S L(2)$ transformations

$$
z_{P} \rightarrow \tilde{z}_{P}=\left(a_{P} z_{P}+b_{P}\right) /\left(c_{P} z_{P}+d_{P}\right) .
$$

Since the Schwarzian derivative $\left\{z_{P}, \tilde{z}_{P}\right\}$ is zero if $z_{P}$ and $\tilde{z}_{P}$ are related as in (A44), this indeterminacy in the $z_{P}$ is limited enough so that the $\hat{f}_{P} \in \hat{\mathscr{S}}_{P}$ are uniquely defined. Then there is a well defined map

$$
f \rightarrow \widehat{f}=\oplus_{P} \hat{f}_{P}
$$

of $\mathscr{S} \rightarrow \tilde{\mathscr{S}}$, and this is an embedding of Lie algebras since

$$
[\hat{f}, \hat{g}]-[\widehat{f, g}]=\frac{c}{24} \sum_{P} \operatorname{Res}_{P} \omega=0 .
$$

Here $\omega$ is the differential form which for any $P$ can be written

$$
\omega=\left(f \frac{d^{3} g}{d z_{P}^{3}}-\frac{d^{3} f}{d z_{P}^{3}} g\right) d z_{P}
$$

in a neighborhood of $P$. It may seem that we are cheating to use a global uniformization (or a global splitting $\mathscr{V}_{0}=L \oplus T$ ) after refusing to use this in the original definition of $\hat{\mathscr{S}}$. The point is that it is essential to define $\hat{\mathscr{S}}$ in an intrinsic, local way, but in investigating its properties one is free to use global methods like the uniformization.

Now we can define the Virasoro Ward identities, that is the identities for the global group $\mathscr{S}$, embedded in $\tilde{\mathscr{S}}$ according to (A45). The "Feynman path integral" should be a linear functional $\int: V \rightarrow k$. The Ward identities are the assertion that for 
$f \in \mathscr{S}$ and $v \in V$

$$
\int \hat{f} \cdot v=0
$$

Since this implies

$$
0=\int([\hat{f}, \hat{g}]-[\widehat{f, g}]) \cdot v,
$$

consistency of the Ward identities depends on the absence of a $c$-number cocycle on the right-hand side of (A46). Such a cocycle being absent, we have accomplished our goal of formulating the Virasoro Ward identities in a global context.

Finally, we briefly return to a situation that we studied in Sect. 1. Let $S$ be a circle that bounds a Riemann surface $\Sigma$. Let $\mathscr{H}_{S}$ be the Hilbert space of states of some conformal field theory formulated on $S$. Let $\left|\Omega_{S}\right\rangle$ be the state obtained by "integrating out $S$." We would like to formulate the Virasoro Ward identities obeyed by the state $\left|\Omega_{S}\right\rangle$.

Let $\mathscr{R}$ be the Lie algebra of complex valued vector fields on $S$, and let $\mathscr{R}_{\Sigma}$ be the subalgebra of $\mathscr{R}$ consisting of vector fields on $S$ that extend holomorphically over $\sum . \mathscr{R}_{\Sigma}$ is a Lie algebra, since if $f$ and $g$ are holomorphic on $\Sigma$, so is $[f, g]=f g^{\prime}-f^{\prime} g$.

If the energy momentum tensor were a conformal field, we would follow precisely the argument that led to Eq. (A59) in Sect. (1) to show that for $f \in \mathscr{R}_{\Sigma}$

$$
\oint_{S} d \theta f T(\theta)\left|\Omega_{\Sigma}\right\rangle=0 .
$$

This is too naive, however, since $\oint d \theta f T(\theta)$ is (because of the conformal anomaly) only well defined modulo a $c$-number; more exactly, $\oint d \theta f T(\theta)$ changes by a $c$-number under a change in the variable $\theta$ by which we parametrize $S$.

What is going on? Recall from (A 59) that the idea in proving a statement like (A 50) is to interpret $f T$ as an "operator valued differential form" and write

$$
0=\int_{\Sigma} \bar{\partial}(f T)=\oint_{S} f T .
$$

Recall our extension of Lie algebras:

$$
0 \rightarrow \Omega^{1} / d \Omega^{0} \rightarrow \hat{\mathscr{S}} \rightarrow \mathscr{S} \rightarrow 0 .
$$

The problem with (A 50) is that $\mathscr{R}_{\Sigma}$ is naturally a subalgebra of $\mathscr{S},{ }^{35}$ but to reason as in (A 51) one must interpret $\mathscr{R}_{\Sigma}$ as a subalgebra of the Lie algebra $\hat{\mathscr{S}}$ of operator valued differential forms. To do this, it is necessary to find a lift $\mathscr{R}_{\Sigma} \rightarrow \hat{\mathscr{S}}$ of $\mathscr{R}$ from $\mathscr{S}$ to $\hat{\mathscr{S}}$. More explicitly, we need a splitting $\mathscr{V}_{0}=L \oplus T$ so that a section $f$ of $T$ can be interpreted as a section $(0, f)$ of $\mathscr{V}_{0}$. A uniformization of $\Sigma$ induces just such a splitting of $\mathscr{V}_{0}$. Using the definition of the energy momentum tensor that would come from a coordinate system corresponding to a uniformization of $\Sigma$, (A 51) and (A 50) are valid.

The existence of any coordinate system in which (A50) is valid implies an interesting statement about the subalgebra $\mathscr{R}_{\Sigma}$ of $\mathscr{R}$. The Lie algebra $\mathscr{R}$ has a nontrivial central extension, corresponding to the Virasoro cocycle (A2). However, the cocycle (A2) must split when restricted to the subalgebra $\mathscr{R}_{\Sigma}$ of $\mathscr{R}$ (that is, upon

${ }^{35}$ In fact, $\mathscr{R}_{\Sigma}$ is precisely the subalgebra of $\mathscr{S}$ consisting of vector fields that are holomorphic on $\Sigma$; recall that $\mathscr{S}$ is a Lie algebra of meromorphic vector fields 
restriction to $\mathscr{R}_{\Sigma}$ it must be possible to eliminate the cocycle by adding $c$-numbers to the operators $\oint f T$ ). For this cocycle must be absent with any definition of $T$ such that (A 50) is valid.

Acknowledgements. I would like to thank M. F. Atiyah, F. Bien, P. Deligne, D. Friedan, D. Husemoller, G. Moore, and especially D. Kazhdan for discussions.

\section{References}

1. Segal, G., Wilson, G.: Loop groups and equations of KdV Type. Pub. IHES 61 (1985)

2. Mulase, M.: J. Differ. Geom. 19, 403 (1984)

3. Shiota, T.: Invent. Math. 83, 333 (1986)

4. Ishibashi, N., Matsuo, Y., Ooguri, H.: Univ. of Tokyo preprint UT-499 (1986)

5. Alvarez-Gaumé, L., Gomez, C., Reina, C.: CERN TH-4641/87

6. Vafa, C.: Operator formulation on Riemann surfaces. Harvard preprint HUTP-87/A008

7. Gelbart, S.S.: Automorphic forms on Adele groups. Princeton, NJ: Princeton University Press 1975

An elementary introduction to the Langlands program. Bull. AMS 10, 177 (1984)

8. Ray, D., Singer, I.: Analytic torsion. Ann. Math. (1973)

9. Quillen, D.: Determinants of Cauchy-Riemann operators over a Riemann surface. Funkts. Anal. Prilozh. 19, 37 (1985) (Funct. Anal. Appl. 19, 31 (1986))

10. Atiyah, M.F., Singer, I.M.: Proc. Natl. Acad. Sci. 81, 2597 (1984)

11. Atiyah, M.F., Patodi, V.K., Singer, I.: Proc. Camb. Philos. Soc. 77, 43 (1975); 78, 405 (1975); 79, 71 (1976)

12. Pressley, A., Segal, G.B.: Loop groups and their representations. Oxford: Oxford University Press 1986

13. Bost, J., Nelson, P.: Spin 1/2 Bosonization on compact surfaces. Phys. Rev. Lett. 57, 795 (1986) Alvarez Gaumé, L., Bost, J.B., Moore, G., Nelson, P., Vafa, C.: Bosonization in arbitrary genus. Phys. Lett. B 178, 41 (1986)

Bosonization on higher genus Riemann surfaces. Commun. Math. Phys. (to appear)

14. Belavin, A.A., Polyakov, A.M., Zamolodchikov, A.B.: Infinite conformal symmetry in twodimensional quantum field theory. Nucl. Phys. B 241, 333 (1984)

15. Friedan, D., Martinec, E., Shenker, S.: Conformal invariance, supersymmetry and string theory. Nucl. Phys. B 271, 93 (1986)

16. Frenkel, I.: Spinor representations of affine Lie algebras. Proc. Natl. Acad. Sci. USA 77, 6303 (1980)

Two constructions of affine Lie algebra representations. J. Funct. Anal. 44, 259 (1981)

17. Garland, H.: The arithmetic theory of loop algebras. J. Algebra 53, 480 (1970)

18. Bowick, M.J., Rajeev, S.G.: MIT preprints (1987)

19. Beilinson, A.A., Manin, Ya.I., Shechtman, V.A.: Localization of the Virasoro and NeveuSchwarz algebras. Preprint (1986)

20. Witten, E.: Noncommutative geometry and string field theory. Nucl. Phys. B 268, 253 (1986)

21. D'Hoker, E., Giddings, S.: Unitarity of the closed bosonic polyakov string. Princeton preprint PUPT-1046 (1987)

22. Strominger, A.: IAS preprints (1986 and 1987)

Communicated by A. Jaffe

Received June 23, 1987 